\title{
Quantum Integrable Model of an Arrangement of Hyperplanes ${ }^{\star}$
}

\author{
Alexander VARCHENKO \\ Department of Mathematics, University of North Carolina at Chapel Hill, \\ Chapel Hill, NC 27599-3250, USA \\ E-mail: anv@email.unc.edu
}

Received July 19, 2010, in final form March 19, 2011; Published online March 28, 2011

doi:10.3842/SIGMA.2011.032

\begin{abstract}
The goal of this paper is to give a geometric construction of the Bethe algebra (of Hamiltonians) of a Gaudin model associated to a simple Lie algebra. More precisely, in this paper a quantum integrable model is assigned to a weighted arrangement of affine hyperplanes. We show (under certain assumptions) that the algebra of Hamiltonians of the model is isomorphic to the algebra of functions on the critical set of the corresponding master function. For a discriminantal arrangement we show (under certain assumptions) that the symmetric part of the algebra of Hamiltonians is isomorphic to the Bethe algebra of the corresponding Gaudin model. It is expected that this correspondence holds in general (without the assumptions). As a byproduct of constructions we show that in a Gaudin model (associated to an arbitrary simple Lie algebra), the Bethe vector, corresponding to an isolated critical point of the master function, is nonzero.
\end{abstract}

Key words: Gaudin model; arrangement of hyperplanes

2010 Mathematics Subject Classification: 82B23; 32S22; 17B81; 81R12

To Sabir Gusein-Zade on the occasion of his 60-th birthday

\section{Contents}

1 Introduction $\quad 3$

1.1 Quantum integrable models and Bethe ansatz . . . . . . . . . . . . . . . . 3

1.2 Gaudin model . . . . . . . . . . . . . . . . . . . . . . . . . 3

1.3 Gaudin model as a semiclassical limit of KZ equations . . . . . . . . . . . . . . . . . . 4

1.4 Bethe ansatz in the Gaudin model . . . . . . . . . . . . . . . . . . . . . . . . . . . . . 5

1.5 Gaudin model and arrangements . . . . . . . . . . . . . . . . . . . . . . . 5

1.6 Bethe algebra . . . . . . . . . . . . . . . . . . . . . . . 5

1.7 Algebra of geometric Hamiltonians . . . . . . . . . . . . . . . . . . . . . . . . 6

1.8 Quantum integrable model of a weighted arrangement (or dynamical theory of arrangements) . . . . . . . . . . . . . . . . . . . . . 6 6

1.9 Bethe ansatz for the quantum integrable model of an arrangement . . . . . . . . . . . . 7

1.10 Geometric interpretation of the algebra of Hamiltonians . . . . . . . . . . . . . . . . . 8

1.11 Byproducts of constructions . . . . . . . . . . . . . . . . . . . . . 8

1.12 Exposition of the material . . . . . . . . . . . . . . . . . . . 8

2 Arrangements 9

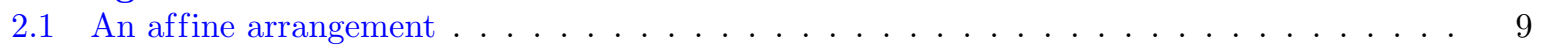

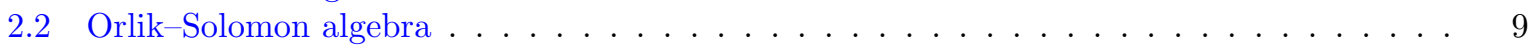

2.3 Weights ................................ 9

*This paper is a contribution to the Special Issue "Relationship of Orthogonal Polynomials and Special Functions with Quantum Groups and Integrable Systems". The full collection is available at http://www.emis.de/journals/SIGMA/OPSF.html 


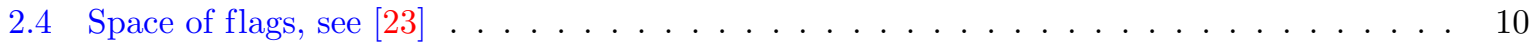

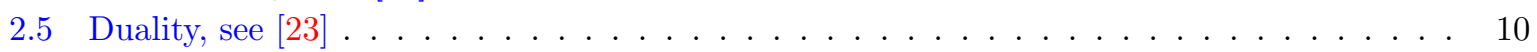

2.6 Contravariant map and form, see $[23] \ldots \ldots \ldots \ldots \ldots \ldots \ldots$

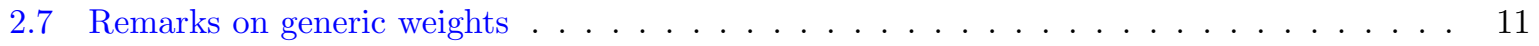

2.8 Orlik-Solomon algebra as an algebra of differential forms $\ldots \ldots \ldots \ldots \ldots$

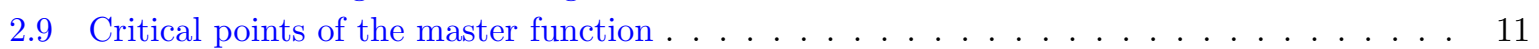

2.10 Special vectors in $\mathcal{F}^{k}(\mathbb{A})$ and canonical element . . . . . . . . . . . . . . . . . . . . . . . . . . .

2.11 Arrangements with normal crossings only $\ldots \ldots \ldots \ldots \ldots \ldots$

2.12 Real structure on $\mathcal{A}^{p}(\mathbb{A})$ and $\mathcal{F}^{p}(\mathbb{A}) \ldots \ldots \ldots \ldots \ldots \ldots \ldots$

2.13 A real arrangement with positive weights . . . . . . . . . . . . . . 14

2.14 Resolution of a hyperplane-like divisor . . . . . . . . . . . . . . . . 15

3 A family of parallelly translated hyperplanes $\quad \mathbf{1 5}$

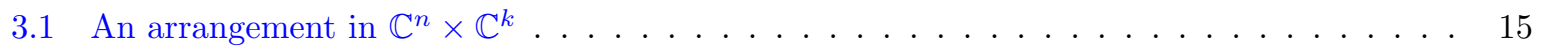

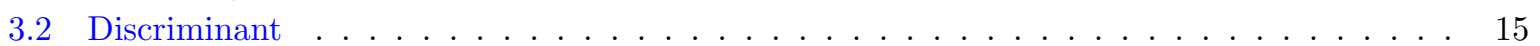

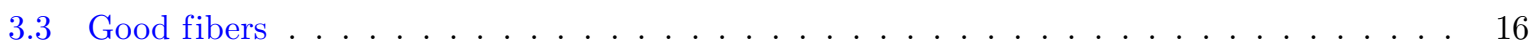

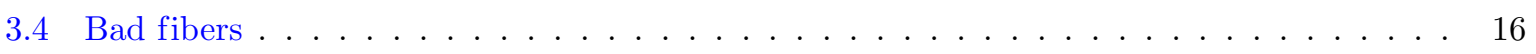

4 Conservation of the number of critical points $\quad 16$

5 Hamiltonians of good fibers $\quad 17$

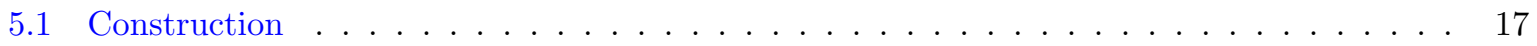

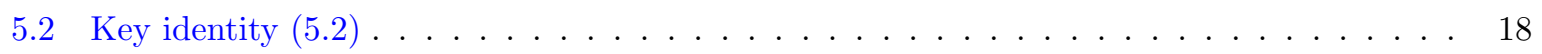

5.3 An application of the key identity $(5.2)$ - proof of Theorem $5.1 \ldots \ldots \ldots$

5.4 Another application of the key identity $(5.2) \ldots \ldots \ldots \ldots \ldots$

5.5 Hamiltonians, critical points and the canonical element . . . . . . . . . . . . . . 21

$6 \quad$ Asymptotic solutions and eigenvectors of Hamiltonians $\quad 21$

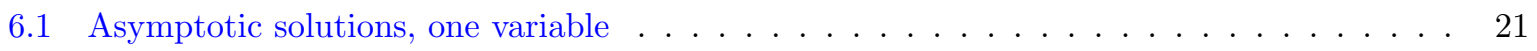

6.2 Critical points of the master function and asymptotic solutions . . . . . . . . . . . 22

7 Hamiltonians of bad fibers $\quad 23$

7.1 Naive geometric Hamiltonians . . . . . . . . . . . . . . . . . . . . 23

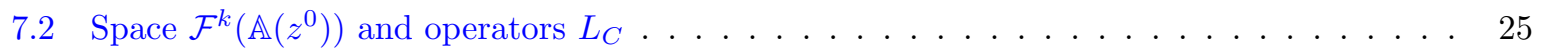

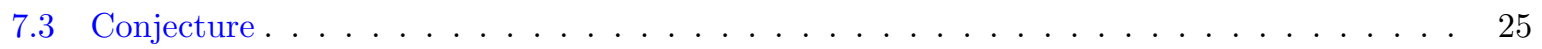

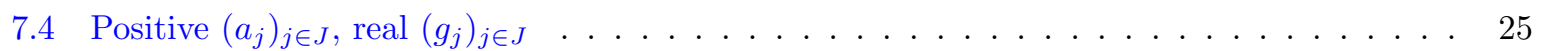

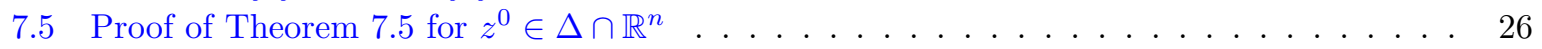

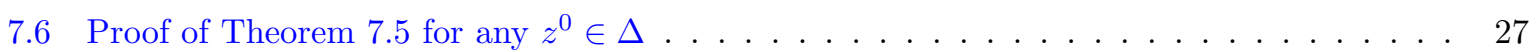

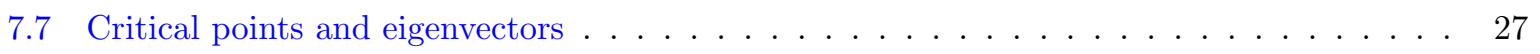

7.8 Hamiltonians, critical points and the canonical element . . . . . . . . . . . . 27

8 Geometric interpretation of the algebra of Hamiltonians $\quad 28$

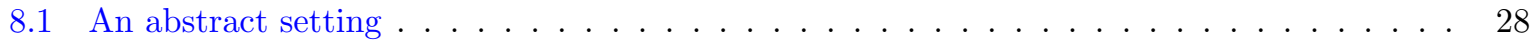

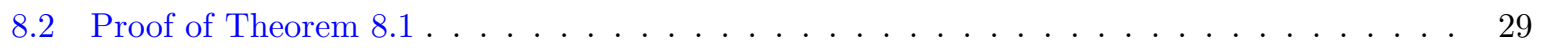

8.3 Remark on maximal commutative subalgebras . . . . . . . . . . . . . . 30

8.4 Interpretation of the algebra of Hamiltonians of good fibers . . . . . . . . . . . . 30

8.5 Interpretation of the algebra of Hamiltonians of bad fibers if Assumption 7.4 is satisfied . 32

9 More on Hamiltonians of bad fibers $\quad 33$

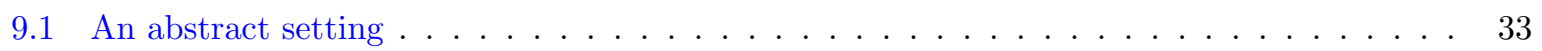

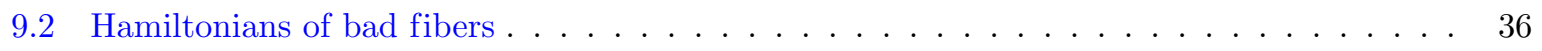

9.3 Remark on critical points of real arrangements . . . . . . . . . . . . . . . . 39 
10 Arrangements with symmetries $\quad 39$

10.1 A family of prediscriminantal arrangements . . . . . . . . . . . . . . 39

10.2 Discriminantal arrangements . . . . . . . . . . . . . . . . . . 40

10.3 Symmetries of the family of prediscriminantal arrangements . . . . . . . . . . . . 41

10.4 The $S_{k}$-action on geometric Hamiltonians $\ldots \ldots \ldots \ldots \ldots$

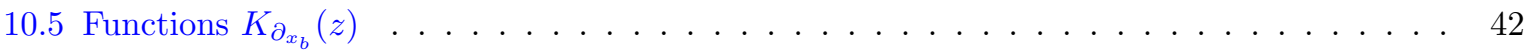

10.6 Naive geometric Hamiltonians on Sing $W^{-}\left(z^{0}\right) \quad \ldots \ldots \ldots \ldots$

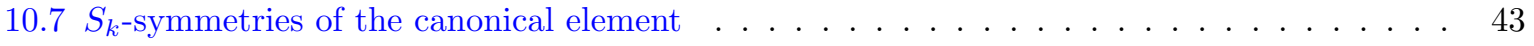

11 Applications to the Bethe ansatz of the Gaudin model $\quad 46$

11.1 Gaudin model . . . . . . . . . . . . . . . . . . . . . . . . 46

11.2 Master function and weight function, $[23] \ldots \ldots \ldots \ldots \ldots$

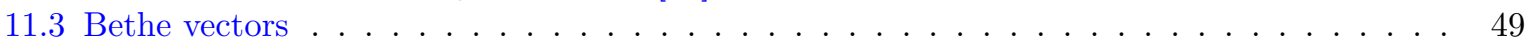

11.4 Identification of Gaudin and naive geometric Hamiltonians _ . . . . . . . . . . . . . 49

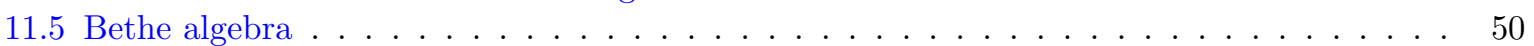

$11.6 \mathfrak{g l}_{r+1}$ Bethe algebra and critical points of the master function $\ldots \ldots \ldots \ldots \ldots$

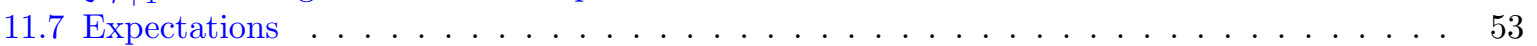

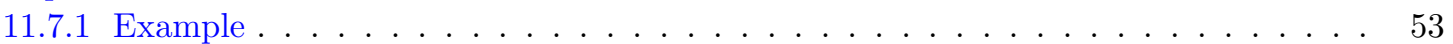

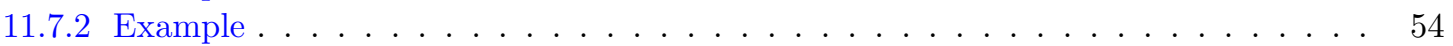

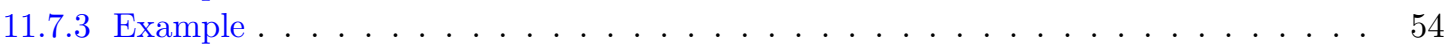

$\begin{array}{ll}\text { References } & 54\end{array}$

\section{Introduction}

\subsection{Quantum integrable models and Bethe ansatz}

A quantum integrable model is a vector space $V$ and an "interesting" collection of commuting linear operators $K_{1}, K_{2}, \ldots: V \rightarrow V$. The operators are called Hamiltonians or transfer matrices or conservation laws. The problem is to find common eigenvectors and eigenvalues.

The Bethe ansatz is a method to diagonalize commuting linear operators. One invents a vector-valued function $v(t)$ of some new parameters $t=\left(t_{1}, \ldots, t_{k}\right)$ and fixes the parameters so that $v(t)$ becomes a common eigenvector of the Hamiltonians. One shows that $v(t) \in V$ is an eigenvector if $t$ satisfies some system of equations,

$$
\Psi_{j}(t)=0, \quad j=1, \ldots, k .
$$

The equations are called the Bethe ansatz equations. The vector $v(t)$ corresponding to a solution of the equations is called a Bethe vector. The method is called the Bethe ansatz method.

\subsection{Gaudin model}

One of the simplest and interesting models is the quantum Gaudin model introduced in [7] and [8]. Choose a simple Lie algebra $\mathfrak{g}$, an orthonormal basis $\left\{J_{a}\right\}$ of $\mathfrak{g}$ with respect to a nondegenerate $\mathfrak{g}$-invariant bilinear form, and a collection of distinct complex numbers $x=\left(x_{1}, \ldots, x_{N}\right)$. Then one defines certain elements of the $N$-th tensor power of the universal enveloping algebra of $\mathfrak{g}$, denoted by $\boldsymbol{K}_{1}(x), \ldots, \boldsymbol{K}_{N}(x) \in(U \mathfrak{g})^{\otimes N}$ and called the Gaudin Hamiltonians,

$$
\boldsymbol{K}_{b}(x)=\sum_{b \neq c} \sum_{a} \frac{J_{a}^{(b)} J_{a}^{(c)}}{x_{b}-x_{c}}, \quad b=1, \ldots, N .
$$

Here we use the standard notation: if $J \in \mathfrak{g}$, then $J^{(i)}=1^{\otimes(i-1)} \otimes J \otimes 1^{\otimes(N-i)}$. 
The Gaudin Hamiltonians commute with each other and commute with the diagonal subalgebra $(U \mathfrak{g})_{\operatorname{diag}} \subset(U \mathfrak{g})^{\otimes N}$,

$$
\left[\boldsymbol{K}_{b}(x), \boldsymbol{K}_{c}(x)\right]=0, \quad\left[\boldsymbol{K}_{b}(x),(U \mathfrak{g})_{\operatorname{diag}}\right]=0 .
$$

Let $V_{\Lambda}$ denote the finite-dimensional irreducible $\mathfrak{g}$-module with highest weight $\Lambda$. Decompose a tensor product $V_{\boldsymbol{\Lambda}}=\otimes_{b=1}^{N} V_{\Lambda_{b}}$ into irreducible $\mathfrak{g}$-modules,

$$
V_{\Lambda}=\oplus_{\Lambda_{\infty}} V_{\Lambda_{\infty}} \otimes W_{\Lambda, \Lambda_{\infty}}
$$

where $W_{\boldsymbol{\Lambda}, \Lambda_{\infty}}$ is the multiplicity space of a representation $V_{\Lambda_{\infty}}$. The Gaudin Hamiltonians act on $V_{\boldsymbol{\Lambda}}$, preserve decomposition (1.2), and by Schur's lemma induce commuting linear operators on each multiplicity space $W=W_{\boldsymbol{\Lambda}, \Lambda_{\infty}}$,

$$
\boldsymbol{K}_{1}(x), \ldots, \boldsymbol{K}_{N}(x): W \rightarrow W .
$$

These commuting linear operators on a multiplicity space constitute the quantum Gaudin model.

Thus, the Gaudin model depends on $\mathfrak{g},($,$) , highest weights \Lambda_{1}, \ldots, \Lambda_{N}, \Lambda_{\infty}$ and complex numbers $x_{1}, \ldots, x_{N}$.

\subsection{Gaudin model as a semiclassical limit of $\mathrm{KZ}$ equations}

On every multiplicity space $W=W_{\boldsymbol{\Lambda}, \Lambda_{\infty}}$ of the tensor product $V_{\boldsymbol{\Lambda}}=\oplus_{\Lambda_{\infty}} V_{\Lambda_{\infty}} \otimes W_{\boldsymbol{\Lambda}, \Lambda_{\infty}}$ one has a system of Knizhnik-Zamolodchikov (KZ) differential equations,

$$
\kappa \frac{\partial I}{\partial x_{b}}(z)=\boldsymbol{K}_{b}(x) I(x), \quad b=1, \ldots, N,
$$

where $x=\left(x_{1}, \ldots, x_{N}\right), I(x) \in W$ is the unknown function, $\boldsymbol{K}_{b}(x)$ are the Gaudin Hamiltonians and $\kappa \in \mathbb{C}^{\times}$is a parameter of the differential equations. KZ equations are equations for conformal blocks in the Wess-Zumino-Novikov-Witten conformal field theory.

For any value of $\kappa, \mathrm{KZ}$ equations define a flat connection on the trivial bundle $\mathbb{C}^{N} \times W \rightarrow \mathbb{C}^{N}$ with singularities over the diagonals. The flatness conditions,

$$
\left[\kappa \frac{\partial}{\partial x_{b}}-\boldsymbol{K}_{b}(x), \kappa \frac{\partial}{\partial x_{c}}-\boldsymbol{K}_{c}(x)\right]=0
$$

in particular, imply the conditions $\left[\boldsymbol{K}_{b}(x), \boldsymbol{K}_{c}(x)\right]=0$, which are the commutativity conditions for the Gaudin Hamiltonians.

Thus, we observe two related problems:

(1) Given a nonzero number $\kappa$, find solutions of the $\mathrm{KZ}$ equations.

(2) Given $x$, find eigenvectors of the Gaudin Hamiltonians.

Problem (2) is a semiclassical limit of problem (1) as $\kappa$ tends to zero. Namely, assume that the $\mathrm{KZ}$ equations have an asymptotic solution of the form

$$
I(x)=e^{P(x) / \kappa}\left(w_{0}(x)+\kappa w_{1}(x)+\kappa^{2} w_{2}(x)+\cdots\right)
$$

as $\kappa \rightarrow 0$. Here $P(x)$ is a scalar function and $w_{0}(x), w_{1}(x), w_{2}(x), \ldots$ are some $W$-valued functions of $x$. Substituting this expression to the KZ equations and equating the leading terms we get

$$
\frac{\partial P}{\partial x_{b}}(x) w_{0}(x)=\boldsymbol{K}_{b}(x) w_{0}(x) .
$$

Hence, for any $x$ and $b$, the vector $w_{0}(x)$ is an eigenvector of the Gaudin Hamiltonian $\boldsymbol{K}_{b}(x)$ with eigenvalue $\frac{\partial P}{\partial x_{b}}(x)$.

Thus, in order to diagonalize the Gaudin Hamiltonians it is enough to construct asymptotic solutions to the $\mathrm{KZ}$ equations. 


\subsection{Bethe ansatz in the Gaudin model}

The Gaudin model has a Bethe ansatz $[2,3,19]$. The Bethe ansatz has special features. Namely,

(i) In the Gaudin model, there exists a function $\Phi\left(t_{1}, \ldots, t_{k}\right)$ (the master function) such that the Bethe ansatz equations (1.1) are the critical point equations for the master function,

$$
\frac{\partial \Phi}{\partial t_{j}}\left(t_{1}, \ldots, t_{k}\right)=0, \quad j=1, \ldots, k .
$$

(ii) In the Gaudin model, the vector space $W$ has a symmetric bilinear form $S$ (the tensor Shapovalov form) and Gaudin Hamiltonians are symmetric operators.

(iii) In the Gaudin model, the Bethe vectors assigned to (properly understood) distinct critical points are orthogonal and the square of the norm of a Bethe vector equals the Hessian of the master function at the corresponding critical point,

$$
S(v(t), v(t))=\operatorname{det}\left(\frac{\partial^{2} \Phi}{\partial t_{i} \partial t_{j}}\right)(t) .
$$

In particular, the Bethe vector corresponding to a nondegenerate critical point is nonzero.

These statements indicate a connection between the Gaudin Hamiltonians and a mysterious master function (which is not present in the definition of the Gaudin model).

One of the goals of this paper is to uncover this mystery and show that the Bethe ansatz can be interpreted as an elementary construction in the theory of arrangements, where the master function is a basic object.

\subsection{Gaudin model and arrangements}

For a weighted arrangement of affine hyperplanes, we will construct (under certain assumptions) a quantum integrable model, that is, a vector space $W$ with a symmetric bilinear form $S$ (called the contravariant form), a collection of commuting symmetric linear operators on $W$ (called the naive geometric Hamiltonians), a master function $\Phi(t)$ and vectors $v(t)$ (called the special vectors or called the values of the canonical element) which are eigenvectors of the linear operators if $t$ is a critical point of the master function.

Then for a given Gaudin model $\left(W, S, \boldsymbol{K}_{1}(x), \boldsymbol{K}_{2}(x), \ldots: W \rightarrow W\right)$, one can find a suitable (discriminantal) arrangement and identify the objects of the Gaudin model with the corresponding objects of the quantum integrable model of the arrangement. After this identification, the master function $\Phi(t)$ and the special vectors $v(t)$ of the arrangement provide the Gaudin model with a Bethe ansatz, that is, with a method to diagonalize the Gaudin Hamiltonians.

\subsection{Bethe algebra}

Let $W_{\boldsymbol{\Lambda}, \Lambda_{\infty}}$ be the vector space of a Gaudin model. It turns out that the subalgebra of End $\left(W_{\boldsymbol{\Lambda}, \Lambda_{\infty}}\right)$ generated by the Gaudin Hamiltonians can be extended to a larger commutative subalgebra called the Bethe algebra. A general construction of the Bethe algebra for a simple Lie algebra $\mathfrak{g}$ is given in [4]. That construction is formulated in terms of the center of the universal enveloping algebra of the corresponding affine Lie algebra $\hat{\mathfrak{g}}$ at the critical level. As a result of that construction, for any $x$ one obtains a commutative subalgebra $B(x) \subset(U \mathfrak{g})^{\otimes N}$ which commutes with the diagonal subalgebra $U \mathfrak{g} \subset(U \mathfrak{g})^{\otimes N}$. To define the Bethe algebra of $V_{\boldsymbol{\Lambda}}$ or of $W_{\boldsymbol{\Lambda}, \Lambda_{\infty}}$ one considers the image of $B\left(x^{0}\right)$ in $\operatorname{End}\left(V_{\boldsymbol{\Lambda}}\right)$ or in $\operatorname{End}\left(W_{\boldsymbol{\Lambda}, \Lambda_{\infty}}\right)$. The Gaudin Hamiltonians $\boldsymbol{K}_{b}(x)$ are elements of the Bethe algebra of $V_{\boldsymbol{\Lambda}}$ or of $W_{\boldsymbol{\Lambda}, \Lambda_{\infty}}$.

The construction of the Bethe algebra in [4] is not explicit and it is not easy to study the Bethe algebra of $W_{\boldsymbol{\Lambda}, \Lambda_{\infty}}$ for a particular Gaudin model. For example, a standard difficult question is if the Bethe algebra of $W_{\boldsymbol{\Lambda}, \Lambda_{\infty}}$ is a maximal commutative subalgebra of $\operatorname{End}\left(W_{\boldsymbol{\Lambda}, \Lambda_{\infty}}\right)$, cf. [12, 5]. 


\subsection{Algebra of geometric Hamiltonians}

In this paper we address the following problem. Is there a geometric construction of the Bethe algebra? For the quantum integrable model $\left(W, S, K_{1}, K_{2}, \ldots: W \rightarrow W\right)$ of a given weighted arrangement, can one define a geometric "Bethe algebra" $A$, which is a maximal commutative subalgebra of $\operatorname{End}(W)$, which contains the naive geometric Hamiltonians $K_{1}, K_{2}, \ldots$, and which can be identified with the Bethe algebra of [4] for discriminantal arrangements?

In this paper (under certain assumptions) we construct an algebra $A$ called the algebra of geometric Hamiltonians and identify it (in certain cases) with the Bethe algebra of the Gaudin model.

\subsection{Quantum integrable model of a weighted arrangement (or dynamical theory of arrangements)}

To define the quantum integrable model of an arrangement we consider in an affine space $\mathbb{C}^{k}$ (with coordinates $t=\left(t_{1}, \ldots, t_{k}\right)$ ) an arrangement of $n$ hyperplanes, $k<n$. Each hyperplane is allowed to move parallelly to itself. The parallel shift of the $i$-th hyperplane is measured by a number $z_{i}$ and for every $z=\left(z_{1}, \ldots, z_{n}\right) \in \mathbb{C}^{n}$ we get in $\mathbb{C}^{k}$ an affine arrangement of hyperplanes $\mathbb{A}(z)=\left(H_{j}(z)\right)$,

$$
H_{j}(z)=\left\{t \in \mathbb{C}^{k} \mid g_{j}(t)+z_{j}=0\right\},
$$

where $g_{j}(t)$ are given linear functions on $\mathbb{C}^{k}$. We assign nonzero numbers $a=\left(a_{j}\right)$ to the hyperplanes of $\mathbb{A}(z)$ (the numbers do not depend on $z$ ) and obtain a family of parallelly translated weighted hyperplanes.

For generic $z \in \mathbb{C}^{n}$, the arrangement $\mathbb{A}(z)$ has normal crossings only. The discriminant $\Delta \subset \mathbb{C}^{n}$ is the subset of all points $z$ such that $\mathbb{A}(z)$ is not with normal crossings only.

The master function $\Phi$ on $\mathbb{C}^{n} \times \mathbb{C}^{k}$ is the function

$$
\Phi(z, t)=\sum_{j} a_{j} \log \left(g_{j}(t)+z_{j}\right)
$$

Let $\mathcal{A}(\mathbb{A}(z))=\oplus_{p=0}^{k} \mathcal{A}^{p}(\mathbb{A}(z))$ be the Orlik-Solomon algebra and $\mathcal{F}(\mathbb{A}(z))=\oplus_{p=0}^{k} \mathcal{F}^{p}(\mathbb{A}(z))$ the dual vector space. We are interested in the top degree components $\mathcal{A}^{k}(\mathbb{A}(z))$ and $\mathcal{F}^{k}(\mathbb{A}(z))$.

The weights $a$ define on $\mathcal{F}^{k}(\mathbb{A}(z))$ a symmetric bilinear form $S^{(a)}$ (called the contravariant form) and a degree-one element $\nu(a)=\sum_{j} a_{j}\left(H_{j}(z)\right) \in \mathcal{A}^{1}(\mathbb{A}(z))$. Denote $\operatorname{Sing} \mathcal{F}^{k}(\mathbb{A}(z)) \subset$ $\mathcal{F}^{k}(\mathbb{A}(z))$ the annihilator of the subspace $\nu(a) \cdot \mathcal{A}^{k-1}(\mathbb{A}(z)) \subset \mathcal{A}^{k}(\mathbb{A}(z))$.

For $z^{1}, z^{2} \in \mathbb{C}^{n}-\Delta$, all combinatorial objects of the arrangements $\mathbb{A}\left(z^{1}\right)$ and $\mathbb{A}\left(z^{2}\right)$ can be canonically identified. In particular, the spaces $\mathcal{F}^{k}\left(\mathbb{A}\left(z^{1}\right)\right), \mathcal{F}^{k}\left(\mathbb{A}\left(z^{2}\right)\right)$ as well as the spaces $\operatorname{Sing} \mathcal{F}^{k}\left(\mathbb{A}\left(z^{1}\right)\right)$, Sing $\mathcal{F}^{k}\left(\mathbb{A}\left(z^{2}\right)\right)$ can be canonically identified. For $z \in \mathbb{C}^{n}-\Delta$, we denote $V=\mathcal{F}^{k}(\mathbb{A}(z))$, Sing $V=\operatorname{Sing} \mathcal{F}^{k}(\mathbb{A}(z))$.

For any nonzero number $\kappa$, the hypergeometric integrals

$$
\int_{\gamma(z)} e^{\Phi(z, t) / \kappa} \omega, \quad \omega \in \mathcal{A}^{k}(\mathbb{A}(z))
$$

define a Gauss-Manin (flat) connection on the trivial bundle $\mathbb{C}^{n} \times \operatorname{Sing} V \rightarrow \mathbb{C}^{n}$ with singularities over the discriminant. The Gauss-Manin differential equations for horizontal sections of the connection on $\mathbb{C}^{n} \times \operatorname{Sing} V \rightarrow \mathbb{C}^{n}$ have the form

$$
\kappa \frac{\partial I}{\partial z_{j}}(z)=K_{j}(z) I(z), \quad j \in J,
$$


where $I(z) \in \operatorname{Sing} V$ is a horizontal section, $K_{j}(z): V \rightarrow V, j \in J$, are suitable linear operators preserving Sing $V$ and independent of $\kappa$. For every $j$, the operator $K_{j}(z)$ is a rational function of $z$ regular on $\mathbb{C}^{n}-\Delta$. Each operator is symmetric with respect to the contravariant form $S^{(a)}$.

These differential equations are our source of quantum integrable models for weighted arrangements. The quantum integrable models are the semiclassical limit of these differential equations similarly to the transition from KZ equations to the Gaudin model in Section 1.6.

The flatness of the connection for all $\kappa$ implies the commutativity of the operators,

$$
\left.\left.K_{i}(z)\right|_{\operatorname{Sing} V} K_{j}(z)\right|_{\operatorname{Sing} V}=\left.\left.K_{j}(z)\right|_{\operatorname{Sing} V} K_{i}(z)\right|_{\operatorname{Sing} V} \quad \text { for all } i, j \text { and } z \in \mathbb{C}^{n}-\Delta .
$$

Let $V^{*}$ be the space dual to $V$. If $M: V \rightarrow V$ is a linear operator, then $M^{*}: V^{*} \rightarrow V^{*}$ denotes the dual operator. Let $W \subset V^{*}$ be the image of $V$ under the map $V \rightarrow V^{*}$ associated with the contravariant form and Sing $W \subset W$ the image of $\operatorname{Sing} V \subset V$. The contravariant form induces on $W$ a nondegenerate symmetric bilinear form, also denoted by $S^{(a)}$.

The operators $K_{j}(z)^{*}$ preserve the subspaces Sing $W \subset W \subset V^{*}$. The operators $\left.K_{j}(z)^{*}\right|_{W}$ : $W \rightarrow W$ are symmetric with respect to the contravariant form. The operators $\left.K_{j}(z)^{*}\right|_{\operatorname{Sing} W}$ : Sing $W \rightarrow \operatorname{Sing} W, j \in J$, commute.

For $z \in \mathbb{C}^{n}-\Delta$, we define the quantum integrable model assigned to $(\mathbb{A}(z), a)$ to be the collection

$$
\left(\operatorname{Sing} W ;\left.S^{(a)}\right|_{\operatorname{Sing} W} ;\left.K_{1}(z)^{*}\right|_{\operatorname{Sing} W}, \ldots,\left.K_{n}(z)^{*}\right|_{\operatorname{Sing} W}: \operatorname{Sing} W \rightarrow \operatorname{Sing} W\right) .
$$

The unital subalgebra of $\operatorname{End}(\operatorname{Sing} W)$ generated by operators $\left.K_{1}(z)^{*}\right|_{\operatorname{Sing} W}, \ldots,\left.K_{n}(z)^{*}\right|_{\operatorname{Sing} W}$ will be called the algebra of geometric Hamiltonians of $(\mathbb{A}(z), a)$.

It is clear that any weighted arrangement with normal crossings only can be realized as a fiber $(\mathbb{A}(z), a)$ of such a construction and, thus, a weighted arrangement with normal crossings only is provided with a quantum integrable model.

If $z^{0}$ is a point of the discriminant, the construction of the quantum integrable model assigned to the arrangement $\left(\mathbb{A}\left(z^{0}\right), a\right)$ is more delicate. The operator valued functions $K_{j}(z)$ may have first order poles at $z^{0}$. We write $K_{j}(z)=K_{j}^{0}(z)+K_{j}^{1}(z)$, where $K_{j}^{0}(z)$ is the polar part at $z^{0}$ and $K_{j}^{1}(z)$ the regular part. By suitably regularizing the operators $K_{j}^{1}\left(z^{0}\right)$, we make them (under certain assumptions) preserve a suitable subspace of $\operatorname{Sing} V$, commute on that subspace and be symmetric with respect to the contravariant form. The algebra of regularized operators $K_{j}^{1}\left(z^{0}\right)$ on that subspace produces the quantum integrable model assigned to the fiber $\left(\mathbb{A}\left(z^{0}\right), a\right)$. It may happen that some linear combinations (with constant coefficients) $K_{\xi}(z)=\sum_{j} \xi_{j} K_{j}(z)$ are regular at $z^{0}$. In this case the operator $K_{\xi}\left(z^{0}\right)$ preserves that subspace and is an element of the algebra of Hamiltonians. In this case the operator $K_{\xi}\left(z^{0}\right)$ is called a naive geometric Hamiltonian. (It is naive in the sense that we don't need to go through the regularization procedure to produce that element of the algebra of Hamiltonians. In that sense, for $z \in \mathbb{C}^{n}-\Delta$ all elements of the algebra of geometric Hamiltonians of the arrangement $(\mathbb{A}(z), a)$ are naive.)

For applications to the Gaudin model one needs a suitable equivariant version of the described construction, the corresponding family of parallelly translated hyperplanes has a symmetry group and the Gaudin model is identified with the skew-symmetric part of the corresponding quantum integrable model of the arrangement.

\subsection{Bethe ansatz for the quantum integrable model of an arrangement}

The Hamiltonians of the model are (suitably regularized) right hand sides of the Gauss-Manin differential equations (1.3). The solutions to the equations are the integrals $\int_{\gamma(z)} e^{\Phi(z, t) / \kappa} \omega$. By taking the semiclassical limit of the integrals as $\kappa$ tends to zero, we obtain eigenvectors of the Hamiltonians, cf. Section 1.3. The eigenvectors of the Hamiltonians are labeled by the critical points of the phase $\Phi$ of the integrals due to the steepest descent method. 


\subsection{Geometric interpretation of the algebra of Hamiltonians}

It turns out that the solutions $\int_{\gamma(z)} e^{\Phi(z, t) / \kappa} \omega$ to equations (1.3) produce more than just eigenvectors of the geometric Hamiltonians. They also produce a geometric interpretation of the whole algebra of geometric Hamiltonians. It turns out that the algebra of geometric Hamiltonians is naturally isomorphic (under certain conditions) to the algebra of functions on the critical set of the master function $\Phi$. The isomorphism is established through the semiclassical limit of the integrals. Moreover, this isomorphism identifies the residue bilinear form on the algebra of functions and the contravariant form of the arrangement.

This geometric interpretation of the algebra of geometric Hamiltonians is motivated by the recent paper [15] where a connection between the algebra of functions on the critical set of the master function and the Bethe algebra of the $\mathfrak{g l}_{r+1}$ Gaudin model is established, cf. [14].

\subsection{Byproducts of constructions}

The general motive of this paper is the interplay between the combinatorially defined linear objects of a weighted arrangement and the critical set of the corresponding master function (which is a nonlinear characteristics of the arrangement). As byproducts of our considerations we get relations between linear and nonlinear characteristics of an arrangement. For example, we prove that the sum of Milnor numbers of the critical points of the master function is not greater than the rank of the contravariant form on $\operatorname{Sing} V$.

As another example of such an interaction we show that in any Gaudin model (associated with any simple Lie algebra) the Bethe vector corresponding to an isolated critical points of the master function is nonzero. That result is known for nondegenerate critical points, see [27], and for the Gaudin models associated with the Lie algebra $\mathfrak{g l}_{r+1}$, see [15].

\subsection{Exposition of the material}

In Section 2, basic facts of the theory of arrangements are collected. The main objects are the space of flags, contravariant form, master function, canonical element.

In Section 3, a family of parallelly translated hyperplanes is introduced. In Section 4, remarks on the conservation of the number of critical points of the master function under deformations are presented.

In Section 5, the Gauss-Manin differential equations are considered. The quantum integrable model of a weighted arrangement with normal crossings only is introduced. The "key identity" (5.2) is formulated.

In Section 6, the asymptotic solutions to the Gauss-Manin differential equations are discussed. In Section 7, the quantum integrable model of any fiber $\left(\mathbb{A}\left(z^{0}\right), a\right), z^{0} \in \Delta$, is defined under assumptions of positivity of weights $\left(a_{j}\right)$ and reality of functions $\left(g_{j}(t)\right)$. A general conjecture is formulated.

In Section 8, it is shown that the algebra of geometric Hamiltonians is isomorphic to the algebra of functions on the critical set of the master function. That fact is proved for any $(\mathbb{A}(z), a), z \in \mathbb{C}^{n}$ under Assumption 7.4 of positivity of $\left(a_{j}\right)$ and reality of $\left(g_{j}(t)\right)$. In Section 9 , more results in this direction are obtained, see Theorems 9.16 and 9.17.

In Section 10, an equivariant version of the algebra of geometric Hamiltonians is introduced and in Section 11 relations with the Gaudin model are described. 


\section{Arrangements}

\subsection{An affine arrangement}

Let $k$ and $n$ be positive integers, $k<n$. Denote $J=\{1, \ldots, n\}$.

Let $\mathbb{A}=\left(H_{j}\right)_{j \in J}$, be an arrangement of $n$ affine hyperplanes in $\mathbb{C}^{k}$. Denote

$$
U=\mathbb{C}^{k}-\cup_{j \in J} H_{j}
$$

the complement. An edge $X_{\alpha} \subset \mathbb{C}^{k}$ of the arrangement $\mathbb{A}$ is a nonempty intersection of some hyperplanes of $\mathbb{A}$. Denote by $J_{\alpha} \subset J$ the subset of indices of all hyperplanes containing $X_{\alpha}$. Denote $l_{\alpha}=\operatorname{codim}_{\mathbb{C}^{k}} X_{\alpha}$.

We always assume that $\mathbb{A}$ is essential, that is, $\mathbb{A}$ has a vertex, an edge which is a point.

An edge is called dense if the subarrangement of all hyperplanes containing it is irreducible: the hyperplanes cannot be partitioned into nonempty sets so that, after a change of coordinates, hyperplanes in different sets are in different coordinates. In particular, each hyperplane of $\mathbb{A}$ is a dense edge.

\subsection{Orlik-Solomon algebra}

Define complex vector spaces $\mathcal{A}^{p}(\mathbb{A}), p=0, \ldots, k$. For $p=0$ set $\mathcal{A}^{p}(\mathbb{A})=\mathbb{C}$. For $p \geqslant 1, \mathcal{A}^{p}(\mathbb{A})$ is generated by symbols $\left(H_{j_{1}}, \ldots, H_{j_{p}}\right)$ with $j_{i} \in J$, such that

(i) $\left(H_{j_{1}}, \ldots, H_{j_{p}}\right)=0$ if $H_{j_{1}}, \ldots, H_{j_{p}}$ are not in general position, that is, if the intersection $H_{j_{1}} \cap \cdots \cap H_{j_{p}}$ is empty or has codimension less than $p$;

(ii) $\left(H_{j_{\sigma(1)}}, \ldots, H_{j_{\sigma(p)}}\right)=(-1)^{|\sigma|}\left(H_{j_{1}}, \ldots, H_{j_{p}}\right)$ for any element $\sigma$ of the symmetric group $S_{p}$;

(iii) $\sum_{i=1}^{p+1}(-1)^{i}\left(H_{j_{1}}, \ldots, \widehat{H}_{j_{i}}, \ldots, H_{j_{p+1}}\right)=0$ for any $(p+1)$-tuple $H_{j_{1}}, \ldots, H_{j_{p+1}}$ of hyperplanes in $\mathbb{A}$ which are not in general position and such that $H_{j_{1}} \cap \cdots \cap H_{j_{p+1}} \neq \varnothing$.

The direct sum $\mathcal{A}(\mathbb{A})=\oplus_{p=1}^{N} \mathcal{A}^{p}(\mathbb{A})$ is an algebra with respect to multiplication

$$
\left(H_{j_{1}}, \ldots, H_{j_{p}}\right) \cdot\left(H_{j_{p+1}}, \ldots, H_{j_{p+q}}\right)=\left(H_{j_{1}}, \ldots, H_{j_{p}}, H_{j_{p+1}}, \ldots, H_{j_{p+q}}\right) .
$$

The algebra is called the Orlik-Solomon algebra of $\mathbb{A}$.

\section{$2.3 \quad$ Weights}

An arrangement $\mathbb{A}$ is weighted if a map $a: J \rightarrow \mathbb{C}, j \mapsto a_{j}$, is given; $a_{j}$ is called the weight of $H_{j}$. For an edge $X_{\alpha}$, define its weight as $a_{\alpha}=\sum_{j \in J_{\alpha}} a_{j}$.

We always assume that $a_{j} \neq 0$ for every $j \in J$.

Define

$$
\nu(a)=\sum_{j \in J} a_{j}\left(H_{j}\right) \in \mathcal{A}^{1}(\mathbb{A}) .
$$

Multiplication by $\nu(a)$ defines a differential

$$
d^{(a)}: \mathcal{A}^{p}(\mathbb{A}) \rightarrow \mathcal{A}^{p+1}(\mathbb{A}), \quad x \mapsto \nu(a) \cdot x,
$$

on $\mathcal{A}(\mathbb{A})$. 


\subsection{Space of flags, see [23]}

For an edge $X_{\alpha}, l_{\alpha}=p$, a flag starting at $X_{\alpha}$ is a sequence

$$
X_{\alpha_{0}} \supset X_{\alpha_{1}} \supset \cdots \supset X_{\alpha_{p}}=X_{\alpha}
$$

of edges such that $l_{\alpha_{j}}=j$ for $j=0, \ldots, p$.

For an edge $X_{\alpha}$, we define $\overline{\mathcal{F}}_{\alpha}$ as the complex vector space with basis vectors

$$
\bar{F}_{\alpha_{0}, \ldots, \alpha_{p}=\alpha}
$$

labeled by the elements of the set of all flags starting at $X_{\alpha}$.

Define $\mathcal{F}_{\alpha}$ as the quotient of $\overline{\mathcal{F}}_{\alpha}$ by the subspace generated by all the vectors of the form

$$
\sum_{X_{\beta}, X_{\alpha_{j-1}} \supset X_{\beta} \supset X_{\alpha_{j+1}}} \bar{F}_{\alpha_{0}, \ldots, \alpha_{j-1}, \beta, \alpha_{j+1}, \ldots, \alpha_{p}=\alpha}
$$

Such a vector is determined by $j \in\{1, \ldots, p-1\}$ and an incomplete flag $X_{\alpha_{0}} \supset \cdots \supset X_{\alpha_{j-1}} \supset$ $X_{\alpha_{j+1}} \supset \cdots \supset X_{\alpha_{p}}=X_{\alpha}$ with $l_{\alpha_{i}}=i$.

Denote by $F_{\alpha_{0}, \ldots, \alpha_{p}}$ the image in $\mathcal{F}_{\alpha}$ of the basis vector $\bar{F}_{\alpha_{0}, \ldots, \alpha_{p}}$. For $p=0, \ldots, k$, set

$$
\mathcal{F}^{p}(\mathbb{A})=\oplus_{X_{\alpha}, l_{\alpha}=p} \mathcal{F}_{\alpha}
$$

\subsection{Duality, see [23]}

The vector spaces $\mathcal{A}^{p}(\mathbb{A})$ and $\mathcal{F}^{p}(\mathbb{A})$ are dual. The pairing $\mathcal{A}^{p}(\mathbb{A}) \otimes \mathcal{F}^{p}(\mathbb{A}) \rightarrow \mathbb{C}$ is defined as follows. For $H_{j_{1}}, \ldots, H_{j_{p}}$ in general position, set $F\left(H_{j_{1}}, \ldots, H_{j_{p}}\right)=F_{\alpha_{0}, \ldots, \alpha_{p}}$ where

$$
X_{\alpha_{0}}=\mathbb{C}^{k}, \quad X_{\alpha_{1}}=H_{j_{1}}, \quad \ldots, \quad X_{\alpha_{p}}=H_{j_{1}} \cap \cdots \cap H_{j_{p}}
$$

Then define $\left\langle\left(H_{j_{1}}, \ldots, H_{j_{p}}\right), F_{\alpha_{0}, \ldots, \alpha_{p}}\right\rangle=(-1)^{|\sigma|}$, if $F_{\alpha_{0}, \ldots, \alpha_{p}}=F\left(H_{j_{\sigma(1)}}, \ldots, H_{j_{\sigma(p)}}\right)$ for some $\sigma \in S_{p}$, and $\left\langle\left(H_{j_{1}}, \ldots, H_{j_{p}}\right), F_{\alpha_{0}, \ldots, \alpha_{p}}\right\rangle=0$ otherwise.

Denote by $\delta^{(a)}: \mathcal{F}^{p}(\mathbb{A}) \rightarrow \mathcal{F}^{p-1}(\mathbb{A})$ the map dual to $d^{(a)}: \mathcal{A}^{p-1}(\mathbb{A}) \rightarrow \mathcal{A}^{p}(\mathbb{A})$. An element $v \in \mathcal{F}^{k}(\mathbb{A})$ is called singular if $\delta^{(a)} v=0$. Denote by

$$
\operatorname{Sing} \mathcal{F}^{k}(\mathbb{A}) \subset \mathcal{F}^{k}(\mathbb{A})
$$

the subspace of all singular vectors.

\subsection{Contravariant map and form, see [23]}

Weights $a$ determine a contravariant map

$$
\mathcal{S}^{(a)}: \mathcal{F}^{p}(\mathbb{A}) \rightarrow \mathcal{A}^{p}(\mathbb{A}), \quad F_{\alpha_{0}, \ldots, \alpha_{p}} \mapsto \sum a_{j_{1}} \cdots a_{j_{p}}\left(H_{j_{1}}, \ldots, H_{j_{p}}\right),
$$

where the sum is taken over all $p$-tuples $\left(H_{j_{1}}, \ldots, H_{j_{p}}\right)$ such that

$$
H_{j_{1}} \supset X_{\alpha_{1}}, \quad \ldots, \quad H_{j_{p}} \supset X_{\alpha_{p}} .
$$

Identifying $\mathcal{A}^{p}(\mathbb{A})$ with $\mathcal{F}^{p}(\mathbb{A})^{*}$, we consider the map as a bilinear form,

$$
S^{(a)}: \mathcal{F}^{p}(\mathbb{A}) \otimes \mathcal{F}^{p}(\mathbb{A}) \rightarrow \mathbb{C} .
$$


The bilinear form is called the contravariant form. The contravariant form is symmetric. For $F_{1}, F_{2} \in \mathcal{F}^{p}(\mathbb{A})$,

$$
S^{(a)}\left(F_{1}, F_{2}\right)=\sum_{\left\{j_{1}, \ldots, j_{p}\right\} \subset J} a_{j_{1}} \cdots a_{j_{p}}\left\langle\left(H_{j_{1}}, \ldots, H_{j_{p}}\right), F_{1}\right\rangle\left\langle\left(H_{j_{1}}, \ldots, H_{j_{p}}\right), F_{2}\right\rangle,
$$

where the sum is over all unordered $p$-element subsets.

The contravariant form was introduced in [23]. It is an analog of the Shapovalov form in representation theory. On relations between the contravariant and Shapovalov forms, see [23] and Section 11.

\subsection{Remarks on generic weights}

Theorem 2.1 ([23]). If weights a are such that none of the dense edges has weight zero, then the contravariant form is nondegenerate.

Theorem 2.2. If weights a are such that none of the dense edges has weight zero, then $H^{p}\left(\mathcal{A}^{*}(\mathbb{A}), d^{(a)}\right)=0$ for $p<k$ and $\operatorname{dim} H^{k}\left(\mathcal{A}^{*}, d^{(a)}\right)=|\chi(U)|$, where $\chi(U)$ is the Euler characteristics of $U$. In particular, these statements hold if all weights are positive.

Proof. The theorem is proved in $[28,18]$. It is also a straightforward corollary of some results in [23]. Namely, in [23] a flag complex $d: \mathcal{F}^{p}(\mathbb{A}) \rightarrow \mathcal{F}^{p+1}(\mathbb{A})$ was considered with the differential defined by formula (2.2.1) in [23]. By [23, Corollary 2.8] the cohomology spaces of that flag complex are trivial in all degrees less than the top degree. In [23], it was also proved that the contravariant map defines a homomorphism of the flag complex to the complex $d^{(a)}: \mathcal{A}^{p}(\mathbb{A}) \rightarrow$ $\mathcal{A}^{p+1}(\mathbb{A})$. Now Theorem 2.2 is a corollary of Theorem 2.1 .

Corollary 2.3. If weights a are such that none of the dense edges has weight zero, then the dimension of $\operatorname{Sing} \mathcal{F}^{k}(\mathbb{A})$ equals $|\chi(U)|$.

\subsection{Orlik-Solomon algebra as an algebra of differential forms}

For $j \in J$, fix a defining equation for the hyperplane $H_{j}, f_{j}=0$, where $f_{j}$ is a polynomial of degree one in variables $t_{1}, \ldots, t_{k}$. Consider the logarithmic differential form $\omega_{j}=d f_{j} / f_{j}$ on $\mathbb{C}^{k}$. Let $\overline{\mathcal{A}}(\mathbb{A})$ be the $\mathbb{C}$-algebra of differential forms generated by 1 and $\omega_{j}, j \in J$. The map $\mathcal{A}(\mathbb{A}) \rightarrow \overline{\mathcal{A}}(\mathbb{A}),\left(H_{j}\right) \mapsto \omega_{j}$, is an isomorphism. We identify $\mathcal{A}(\mathbb{A})$ and $\overline{\mathcal{A}}(\mathbb{A})$.

\subsection{Critical points of the master function}

Given weights $a: J \rightarrow \mathbb{C}$, define the (multivalued) master function $\Phi: U \rightarrow \mathbb{C}$,

$$
\Phi=\Phi_{\mathbb{A}, a}=\sum_{j \in J} a_{j} \log f_{j}
$$

Usually the function $e^{\Phi}=\prod_{j} f_{j}^{a_{j}}$ is called the master function, see [25, 26, 27], but it is more convenient to work with definition (2.1).

A point $t \in U$ is a critical point of $\Phi$ if $\left.d \Phi\right|_{t}=0$. We can rewrite this equation as $\left.\nu(a)\right|_{t}=0$ since

$$
\nu(a)=d \Phi
$$

Theorem 2.4 ([26, 17, 21]). For generic exponents a all critical points of $\Phi$ are nondegenerate and the number of critical points equals $|\chi(U)|$. 
Denote $\mathbb{C}(t)_{U}$ the algebra of rational functions on $\mathbb{C}^{k}$ regular on $U$ and

$$
I_{\Phi}=\left\langle\frac{\partial \Phi}{\partial t_{i}} \mid i=1, \ldots, k\right\rangle \subset \mathbb{C}(t)_{U}
$$

the ideal generated by first derivatives of $\Phi$. Let $A_{\Phi}=\mathbb{C}(t)_{U} / I_{\Phi}$ be the algebra of functions on the critical set and [ ]: $\mathbb{C}(t)_{U} \rightarrow A_{\Phi}, f \mapsto[f]$, the canonical homomorphism.

If all critical points are isolated, then the critical set is finite and the algebra $A_{\Phi}$ is finitedimensional. In that case, $A_{\Phi}$ is the direct sum of local algebras corresponding to points $p$ of the critical set,

$$
A_{\Phi}=\oplus_{p} A_{p, \Phi}
$$

The local algebra $A_{p, \Phi}$ can be defined as the quotient of the algebra of germs at $p$ of holomorphic functions modulo the ideal $I_{p, \Phi}$ generated first derivatives of $\Phi$. Denote by $\mathfrak{m}_{p} \subset A_{p, \Phi}$ the maximal ideal generated by germs of functions equal to zero at $p$.

Lemma 2.5. The elements $\left[1 / f_{j}\right], j \in J$, generate $A_{\Phi}$.

Proof. If $H_{j_{1}}, \ldots, H_{j_{k}}$ intersect transversally, then $1 / f_{j_{1}}, \ldots, 1 / f_{j_{k}}$ form a coordinate system on $U$. This remark proves the lemma.

Define a rational function Hess ${ }^{(a)}: \mathbb{C}^{k} \rightarrow \mathbb{C}$, regular on $U$, by the formula

$$
\operatorname{Hess}^{(a)}(t)=\operatorname{det}_{1 \leqslant i, j \leqslant k}\left(\frac{\partial^{2} \Phi}{\partial t_{i} \partial t_{j}}\right)(t)
$$

The function is called the Hessian of $\Phi$.

Let $p$ be an isolated critical point of $\Phi$. Denote by $\left[\operatorname{Hess}^{(\mathrm{a})}\right]_{p}$ the image of the Hessian in $A_{p, \Phi}$. It is known that the image is nonzero and the one-dimensional subspace $\mathbb{C}\left[\operatorname{Hess}^{(\mathrm{a})}\right]_{p} \subset A_{p, \Phi}$ is the annihilating ideal of the maximal ideal $\mathfrak{m}_{p} \subset A_{p, \Phi}$.

Let $\rho_{p}: A_{p, \Phi} \rightarrow \mathbb{C}$, be the Grothendieck residue,

$$
f \mapsto \frac{1}{(2 \pi i)^{k}} \operatorname{Res}_{p} \frac{f}{\prod_{i=1}^{k} \frac{\partial \Phi}{\partial t_{i}}}=\frac{1}{(2 \pi i)^{k}} \int_{\Gamma_{p}} \frac{f d t_{1} \wedge \cdots \wedge d t_{k}}{\prod_{s=1}^{k} \frac{\partial \Phi}{\partial t_{i}}},
$$

where $\Gamma_{p}=\left\{\left(t_{1}, \ldots, t_{k}\right)|| \frac{\partial \Phi}{\partial t_{i}} \mid=\epsilon_{i}, i=1, \ldots, k\right\}$ is the real $k$-cycle oriented by the condition

$$
d \arg \frac{\partial \Phi}{\partial t_{1}} \wedge \cdots \wedge d \arg \frac{\partial \Phi}{\partial t_{k}} \geqslant 0
$$

here $\epsilon_{s}$ are sufficiently small positive real numbers, see [9].

It is known that $\rho_{p}:\left[\operatorname{Hess}^{(\mathrm{a})}\right]_{p} \mapsto \mu_{p}$, where $\mu_{p}=\operatorname{dim}_{\mathbb{C}} A_{p, \Phi}$ is the Milnor number of the critical point $p$. Let $(,)_{p}$ be the residue bilinear form,

$$
(f, g)_{p}=\rho_{p}(f g)
$$

That form is nondegenerate. 


\subsection{Special vectors in $\mathcal{F}^{k}(\mathbb{A})$ and canonical element}

A differential top degree form $\eta \in \mathcal{A}^{k}(\mathbb{A})$ can be written as

$$
\eta=f d t_{1} \wedge \cdots \wedge d t_{k}
$$

where $f$ is a rational function on $\mathbb{C}^{k}$, regular on $U$.

Define a rational map $v: \mathbb{C}^{k} \rightarrow \mathcal{F}^{k}(\mathbb{A})$ regular on $U$. For $t \in U$, set $v(t)$ to be the element of $\mathcal{F}^{k}(\mathbb{A})$ such that

$$
\langle\eta, v(t)\rangle=f(t) \quad \text { for any } \eta \in \mathcal{A}^{k}(\mathbb{A}) .
$$

The map $v$ is called the specialization map and its value $v(t)$ is called the special vector at $t \in U$, see [27].

Let $\left(F_{m}\right)_{m \in M}$ be a basis of $\mathcal{F}^{k}(\mathbb{A})$ and $\left(H^{m}\right)_{m \in M} \subset \mathcal{A}^{k}(\mathbb{A})$ the dual basis. Consider the element $\sum_{m} H^{m} \otimes F_{m} \in \mathcal{A}^{k}(\mathbb{A}) \otimes \mathcal{F}^{k}(\mathbb{A})$. We have $H^{m}=f^{m} d t_{1} \wedge \cdots \wedge d t_{k}$ for some $f^{m} \in \mathbb{C}(t)_{U}$. The element

$$
E=\sum_{m \in M} f^{m} \otimes F_{m} \in \mathbb{C}(t)_{U} \otimes \mathcal{F}^{k}(\mathbb{A})
$$

will be called the canonical element of the arrangement $\mathbb{A}$. It does not depend on the choice of the basis $\left(F_{m}\right)_{m \in M}$.

For any $t \in U$, we have

$$
v(t)=\sum_{m \in M} f^{m}(t) F_{m}
$$

Denote by $[E]$ the image of the canonical element in $A_{\Phi} \otimes \mathcal{F}^{k}(\mathbb{A})$.

Lemma 2.6. We have $[E] \in A_{\Phi} \otimes \operatorname{Sing} \mathcal{F}^{k}(\mathbb{A})$.

Proof. By formula $(2.2)$, if $e \in d^{(a)} \cdot \mathcal{A}(\mathbb{A})^{k-1}$, then $\langle 1 \otimes e, E\rangle \in I_{\Phi}$. Hence

$$
\langle 1 \otimes e,[E]\rangle=0
$$

in $A_{\Phi}$. Let $e_{1}, \ldots, e_{l}$ be a basis of $d^{(a)} \cdot \mathcal{A}^{k-1}(\mathbb{A})$. Extend it to a basis $e_{1}, \ldots, e_{l}, e_{l+1}, \ldots, e_{|M|}$ of $\mathcal{A}^{k}(\mathbb{A})$. Let $e^{1}, \ldots, e^{l}, e^{l+1}, \ldots, e^{|M|}$ be the dual basis of $\mathcal{F}^{k}(\mathbb{A})$. Then $e^{l+1}, \ldots, e^{|M|}$ is a basis of $\operatorname{Sing} \mathcal{F}^{k}(\mathbb{A})$. Let $[E]=\sum_{i=1}^{|M|}\left[g^{i}\right] \otimes e^{i}$ for some $\left[g^{i}\right] \in A_{\Phi}$. By $(2.4)$ we have $\left[g^{i}\right]=0$ for all $i \leqslant l$.

Theorem $2.7([27])$. A point $t \in U$ is a critical point of $\Phi$, if and only if the special vector $v(t)$ is a singular vector.

Proof. The theorem follows from Lemma 2.6.

Theorem $2.8([27])$.

(i) For any $t \in U$,

$$
S^{(a)}(v(t), v(t))=(-1)^{k} \operatorname{Hess}^{(\mathrm{a})}(t) .
$$

(ii) If $t^{1}, t^{2} \in U$ are different isolated critical points of $\Phi$, then the special singular vectors $v\left(t^{1}\right), v\left(t^{2}\right)$ are orthogonal,

$$
S^{(a)}\left(v\left(t^{1}\right), v\left(t^{2}\right)\right)=0 .
$$




\subsection{Arrangements with normal crossings only}

An essential arrangement $\mathbb{A}$ is with normal crossings only, if exactly $k$ hyperplanes meet at every vertex of $\mathbb{A}$. Assume that $\mathbb{A}$ is an essential arrangement with normal crossings only.

A subset $\left\{j_{1}, \ldots, j_{p}\right\} \subset J$ will be called independent if the hyperplanes $H_{j_{1}}, \ldots, H_{j_{p}}$ intersect transversally.

A basis of $\mathcal{A}^{p}(\mathbb{A})$ is formed by $\left(H_{j_{1}}, \ldots, H_{j_{p}}\right)$ where $\left\{j_{1}<\cdots<j_{p}\right\}$ are independent ordered $p$-element subsets of $J$. The dual basis of $\mathcal{F}^{p}(\mathbb{A})$ is formed by the corresponding vectors $F\left(H_{j_{1}}, \ldots, H_{j_{p}}\right)$. These bases of $\mathcal{A}^{p}(\mathbb{A})$ and $\mathcal{F}^{p}(\mathbb{A})$ will be called standard.

In $\mathcal{F}^{p}(\mathbb{A})$ we have

$$
F\left(H_{j_{1}}, \ldots, H_{j_{p}}\right)=(-1)^{|\sigma|} F\left(H_{j_{\sigma(1)}}, \ldots, H_{j_{\sigma(p)}}\right)
$$

for any permutation $\sigma \in S_{p}$.

For an independent subset $\left\{j_{1}, \ldots, j_{p}\right\}$, we have

$$
S^{(a)}\left(F\left(H_{j_{1}}, \ldots, H_{j_{p}}\right), F\left(H_{j_{1}}, \ldots, H_{j_{p}}\right)\right)=a_{j_{1}} \cdots a_{j_{p}}
$$

and

$$
S^{(a)}\left(F\left(H_{j_{1}}, \ldots, H_{j_{p}}\right), F\left(H_{i_{1}}, \ldots, H_{i_{k}}\right)\right)=0
$$

for distinct elements of the standard basis.

\subsection{Real structure on $\mathcal{A}^{p}(\mathbb{A})$ and $\mathcal{F}^{p}(\mathbb{A})$}

We have defined $\mathcal{A}^{p}(\mathbb{A})$ and $\mathcal{F}^{p}(\mathbb{A})$ as vector spaces over $\mathbb{C}$. But one can define the corresponding spaces over the field $\mathbb{R}$ so that $\mathcal{A}^{p}(\mathbb{A})=\mathcal{A}^{p}(\mathbb{A})_{\mathbb{R}} \otimes_{\mathbb{R}} \mathbb{C}$ and $\mathcal{F}^{p}(\mathbb{A})=\mathcal{F}^{p}(\mathbb{A})_{\mathbb{R}} \otimes_{\mathbb{R}} \mathbb{C}$. If all weights $a$ are real, then the differential $d^{(a)}: \mathcal{A}^{p}(\mathbb{A}) \rightarrow \mathcal{A}^{p+1}(\mathbb{A})$ preserves the real subspaces and one can define the subspace of singular vectors $\operatorname{Sing} \mathcal{F}^{k}(\mathbb{A})_{\mathbb{R}} \subset \mathcal{F}^{k}(\mathbb{A})_{\mathbb{R}}$ so that $\operatorname{Sing} \mathcal{F}^{k}(\mathbb{A})=$ $\operatorname{Sing} \mathcal{F}^{k}(\mathbb{A})_{\mathbb{R}} \otimes_{\mathbb{R}} \mathbb{C}$.

\subsection{A real arrangement with positive weights}

Let $t_{1}, \ldots, t_{k}$ be standard coordinates on $\mathbb{C}^{k}$. Assume that every polynomial $f_{j}, j \in J$, has real coefficients,

$$
f_{j}=z_{j}+b_{j}^{1} t_{1}+\cdots+b_{j}^{k} t_{k}
$$

where $z_{j}, b_{j}^{i}$ are real numbers.

Denote $U_{\mathbb{R}}=U \cap \mathbb{R}^{k}$. Let $U_{\mathbb{R}}=\cup_{\alpha} D_{\alpha}$ be the decomposition into the union of connected components. Each connected component is a convex polytope. It is known that the number of bounded connected components equals $|\chi(U)|$, see [29].

Theorem 2.9 ([26]). Assume that weights $\left(a_{j}\right)_{j \in J}$ are positive. Then the union of all critical points of the master function $\Phi_{\mathbb{A}, a}$ is contained in the union of all bounded components of $U_{\mathbb{R}}$. Each bounded component contains exactly one critical point. All critical points are nondegenerate.

Corollary 2.10. Under assumptions of Theorem 2.9 let $t^{1}, \ldots, t^{d} \in U_{\mathbb{R}}$ be a list of all distinct critical points of the master function $\Phi_{\mathbb{A}, a}$. Then the corresponding special vectors $v\left(t^{1}\right), \ldots, v\left(t^{d}\right)$ form a basis of $\operatorname{Sing} \mathcal{F}^{k}(\mathbb{A})_{\mathbb{R}}$. That basis is orthogonal with respect to the contravariant form $S^{(a)}$.

Note that the contravariant form on $\operatorname{Sing} \mathcal{F}^{k}(\mathbb{A})_{\mathbb{R}}$ is positive definite. 


\subsection{Resolution of a hyperplane-like divisor}

Let $Y$ be a smooth complex compact manifold of dimension $k, D$ a divisor. The divisor $D$ is hyperplane-like if $Y$ can be covered by coordinate charts such that in each chart $D$ is a union of hyperplanes. Such charts will be called linearizing. Let $D$ be a hyperplane-like divisor, $U$ be a linearizing chart. A local edge of $D$ in $U$ is any nonempty irreducible intersection in $U$ of hyperplanes of $D$ in $U$. An edge of $D$ is the maximal analytic continuation in $Y$ of a local edge. Any edge is an immersed submanifold in $Y$. An edge is called dense if it is locally dense. For $0 \leqslant i \leqslant k-2$, let $\mathcal{L}_{i}$ be the collection of all dense edges of $D$ of dimension $i$. The following theorem is essentially contained in Section 10.8 of [25].

Theorem 2.11 ([22]). Let $W_{0}=Y$. Let $\pi_{1}: W_{1} \rightarrow W_{0}$ be the blow up along points in $\mathcal{L}_{0}$. In general, for $1 \leqslant s \leqslant k-1$, let $\pi_{s}: W_{s} \rightarrow W_{s-1}$ be the blow up along the proper transforms of the $(s-1)$-dimensional dense edges in $\mathcal{L}_{s-1}$ under $\pi_{1} \cdots \pi_{s-1}$. Let $\pi=\pi_{1} \cdots \pi_{k-1}$. Then $W=W_{n-1}$ is nonsingular and $\pi^{-1}(D)$ has normal crossings.

\section{A family of parallelly translated hyperplanes}

\subsection{An arrangement in $\mathbb{C}^{n} \times \mathbb{C}^{k}$}

Recall that $J=\{1, \ldots, n\}$. Consider $\mathbb{C}^{k}$ with coordinates $t_{1}, \ldots, t_{k}, \mathbb{C}^{n}$ with coordinates $z_{1}, \ldots, z_{n}$, the projection $\mathbb{C}^{n} \times \mathbb{C}^{k} \rightarrow \mathbb{C}^{n}$.

Fix $n$ nonzero linear functions on $\mathbb{C}^{k}$,

$$
g_{j}=b_{j}^{1} t_{1}+\cdots+b_{j}^{k} t_{k}, \quad j \in J,
$$

where $b_{j}^{i} \in \mathbb{C}$. Define $n$ linear functions on $\mathbb{C}^{n} \times \mathbb{C}^{k}$,

$$
f_{j}=z_{j}+g_{j}=z_{j}+b_{j}^{1} t_{1}+\cdots+b_{j}^{k} t_{k}, \quad j \in J .
$$

In $\mathbb{C}^{n} \times \mathbb{C}^{k}$ define an arrangement

$$
\tilde{\mathbb{A}}=\left\{\tilde{H}_{j} \mid f_{j}=0, j \in J\right\} .
$$

Denote $\tilde{U}=\mathbb{C}^{n} \times \mathbb{C}^{k}-\cup_{j \in J} \tilde{H}_{j}$.

For every fixed $z=\left(z_{1}, \ldots, z_{n}\right)$ the arrangement $\tilde{\mathbb{A}}$ induces an arrangement $\mathbb{A}(z)$ in the fiber over $z$ of the projection. We identify every fiber with $\mathbb{C}^{k}$. Then $\mathbb{A}(z)$ consists of hyperplanes $H_{j}(z), j \in J$, defined in $\mathbb{C}^{k}$ by the same equations $f_{j}=0$. Denote

$$
U(\mathbb{A}(z))=\mathbb{C}^{k}-\cup_{j \in J} H_{j}(z),
$$

the complement to the arrangement $\mathbb{A}(z)$.

In the rest of the paper we assume that for any $z$ the arrangement $\mathbb{A}(z)$ has a vertex. This means that the span of $\left(g_{j}\right)_{j \in J}$ is $k$-dimensional.

A point $z \in \mathbb{C}^{n}$ will be called good if $\mathbb{A}(z)$ has normal crossings only. Good points form the complement in $\mathbb{C}^{n}$ to the union of suitable hyperplanes called the discriminant.

\subsection{Discriminant}

The collection $\left(g_{j}\right)_{j \in J}$ induces a matroid structure on $J$. A subset $C=\left\{i_{1}, \ldots, i_{r}\right\} \subset J$ is a circuit if $\left(g_{i}\right)_{i \in C}$ are linearly dependent but any proper subset of $C$ gives linearly independent $g_{i}$ 's.

For a circuit $C=\left\{i_{1}, \ldots, i_{r}\right\}$, let $\left(\lambda_{i}^{C}\right)_{i \in C}$ be a nonzero collection of complex numbers such that $\sum_{i \in C} \lambda_{i}^{C} g_{i}=0$. Such a collection is unique up to multiplication by a nonzero number. 
For every circuit $C$ fix such a collection and denote $f_{C}=\sum_{i \in C} \lambda_{i}^{C} z_{i}$. The equation $f_{C}=0$ defines a hyperplane $H_{C}$ in $\mathbb{C}^{n}$. It is convenient to assume that $\lambda_{i}^{C}=0$ for $i \in J-C$ and write $f_{C}=\sum_{i \in J} \lambda_{i}^{C} z_{i}$.

For any $z \in \mathbb{C}^{n}$, the hyperplanes $\left(H_{i}(z)\right)_{i \in C}$ in $\mathbb{C}^{k}$ have nonempty intersection if and only if $z \in H_{C}$. If $z \in H_{C}$, then the intersection has codimension $r-1$ in $\mathbb{C}^{k}$.

Denote by $\mathfrak{C}$ the set of all circuits in $J$. Denote $\Delta=\cup_{C \in \mathfrak{C}} H_{C}$.

Lemma 3.1. The arrangement $\mathbb{A}(z)$ in $\mathbb{C}^{k}$ has normal crossings only, if and only if $z \in \mathbb{C}^{n}-\Delta$.

Remark 3.2. If all linear functions $g_{j}, j \in J$, are real, then for any circuit $C \in \mathfrak{C}$ the numbers $\left(\lambda_{i}^{C}\right)_{i \in C}$ can be chosen to be real. Therefore, in that case every hyperplane $H_{C}$ is real.

\subsection{Good fibers}

For any $z^{1}, z^{2} \in \mathbb{C}^{n}-\Delta$, the spaces $\mathcal{F}^{p}\left(\mathbb{A}\left(z^{1}\right)\right), \mathcal{F}^{p}\left(\mathbb{A}\left(z^{2}\right)\right)$ are canonically identified. Namely, a vector $F\left(H_{j_{1}}\left(z^{1}\right), \ldots, H_{j_{p}}\left(z^{1}\right)\right)$ of the first space is identified with the vector $F\left(H_{j_{1}}\left(z^{2}\right), \ldots\right.$, $\left.H_{j_{p}}\left(z^{2}\right)\right)$ of the second.

Assume that weights $a=\left(a_{j}\right)_{j \in J}$ are given and all of them are nonzero. Then each arrangement $\mathbb{A}(z)$ is weighted. The identification of spaces $\mathcal{F}^{p}\left(\mathbb{A}\left(z^{1}\right)\right), \mathcal{F}^{p}\left(\mathbb{A}\left(z^{2}\right)\right)$ for $z^{1}, z^{2} \in \mathbb{C}^{n}-\Delta$ identifies the corresponding subspaces Sing $\mathcal{F}^{k}\left(\mathbb{A}\left(z^{1}\right)\right)$, Sing $\mathcal{F}^{k}\left(\mathbb{A}\left(z^{2}\right)\right)$ and contravariant forms.

For a point $z \in \mathbb{C}^{n}-\Delta$, denote $V=\mathcal{F}^{k}(\mathbb{A}(z))$, Sing $V=\operatorname{Sing} \mathcal{F}^{k}(\mathbb{A}(z))$. The triple $\left(V\right.$, Sing $\left.V, S^{(a)}\right)$ does not depend on $z \in \mathbb{C}^{n}-\Delta$ under the above identification.

\subsection{Bad fibers}

Points of $\Delta \subset \mathbb{C}^{n}$ will be called bad.

Let $z^{0} \in \Delta$ and $z \in \mathbb{C}^{n}-\Delta$. By definition, for any $p$ the space $\mathcal{A}^{p}\left(\mathbb{A}\left(z^{0}\right)\right)$ is obtained from $\mathcal{A}^{p}(\mathbb{A}(z))$ by adding new relations. Hence $\mathcal{A}^{k}\left(\mathbb{A}\left(z^{0}\right)\right)$ is canonically identified with a quotient space of $V^{*}=\mathcal{A}^{k}(\mathbb{A}(z))$ and $\mathcal{F}^{p}\left(\mathbb{A}\left(z^{0}\right)\right)$ is canonically identified with a subspace of $V=\mathcal{F}^{p}(\mathbb{A}(z))$.

Let us consider $\mathcal{F}^{k}\left(z^{0}\right)$ as a subspace of $V$. Let $\left.S^{(a)}\right|_{\mathcal{F}^{k}\left(z^{0}\right)}$ be the restriction of the contravariant form on $V$ to that subspace. Let $S^{(a)}\left(z^{0}\right)$ be the contravariant form on $\mathcal{F}^{k}\left(\mathbb{A}\left(z^{0}\right)\right)$ of the arrangement $\mathbb{A}\left(z^{0}\right)$.

Lemma 3.3. Under the above identifications, $\left.S^{(a)}\right|_{\mathcal{F}^{k}\left(z^{0}\right)}=S^{(a)}\left(z^{0}\right)$.

\section{Conservation of the number of critical points}

Let $\mathbb{A}=\left(H_{j}\right)_{j \in J}$ be an essential arrangement in $\mathbb{C}^{k}$ with weights $a$. Consider its compactification in the projective space $\mathbb{P}^{k}$ containing $\mathbb{C}^{k}$. Assign the weight $a_{\infty}=-\sum_{j \in J} a_{j}$ to the hyperplane $H_{\infty}=\mathbb{P}^{k}-\mathbb{C}^{k}$ and denote by $\mathbb{A}^{\vee}$ the arrangement $\left(H_{j}\right)_{j \in J \cup \infty}$ in $\mathbb{P}^{k}$.

The weighted arrangement $(\mathbb{A}, a)$ will be called unbalanced if the weight of any dense edge of $\mathbb{A}^{\vee}$ is nonzero.

For example, if all weights $\left(a_{j}\right)_{j \in J}$ are positive, then the weighted arrangement $(\mathbb{A}, a)$ is unbalanced. Clearly, the unbalanced weights form a Zarisky open subset in the space of all weights of $\mathbb{A}$.

Lemma 4.1. If $(\mathbb{A}, a)$ is unbalanced, then all critical points of the master function of the weighted arrangement $(\mathbb{A}, a)$ are isolated. 
Proof. Let $\pi: W \rightarrow \mathbb{P}^{k}$ be the resolution (described in Theorem 2.11) of singularities of the divisor $D=\cup_{j \in J \cup \infty} H_{j}$. Let $\Phi_{a}$ be the master function of $(\mathbb{A}, a)$. Then locally on $W$ the function $\pi^{-1} \Phi_{a}$ has the form

$$
\pi^{-1} \Phi_{a}=\sum_{i=1}^{m} \alpha_{i} \log u_{i}+\log \phi\left(u_{1}, \ldots, u_{k}\right), \quad \phi(0, \ldots, 0) \neq 0 .
$$

Here $u_{1}, \ldots, u_{k}$ are local coordinates, $0 \leqslant m \leqslant k$, the function $\phi\left(u_{1}, \ldots, u_{k}\right)$ is holomorphic at $u=0$, the equation $u_{1} \cdots u_{m}=0$ defines $\pi^{-1}(D)$ in this chart. If the image of a divisor $u_{i}=0$, $1 \leqslant i \leqslant m$, under the map $\pi$ is an $s$-dimensional piece of an $s$-dimensional dense edge of $\mathbb{A}^{\vee}$, then $\alpha_{i}$ equals the weight of that edge. In particular, $\alpha_{i}, i=1, \ldots, m$, are all nonzero.

Let $U(\mathbb{A})=\mathbb{C}^{k}-\cup_{j \in J} H_{j}$. The critical point equations of $\pi^{-1} \Phi_{a}$ on $\pi^{-1}(U(\mathbb{A}))$ are

$$
\begin{aligned}
& \frac{\alpha_{i}}{u_{i}}+\frac{1}{\phi(u)} \frac{\partial \phi}{\partial u_{i}}(u)=0, \quad i=1, \ldots, m, \\
& \frac{1}{\phi(u)} \frac{\partial \phi}{\partial u_{i}}(u)=0, \quad i=m+1, \ldots, k .
\end{aligned}
$$

If the critical set of $\pi^{-1} \Phi_{a}$ on $\pi^{-1}(U(\mathbb{A}))$ is infinite, then it contains an algebraic curve. The closure of that curve must intersect $\pi^{-1}(D)$. But equations (4.1) show that this is impossible.

Denote by $\mu(\mathbb{A}, a)$ the sum of Milnor numbers of all of the critical points of $\Phi_{a}$ on $U(\mathbb{A})$.

Lemma 4.2. If $(\mathbb{A}, a)$ is unbalanced, then $\mu(\mathbb{A}, a)=|\chi(U)|$.

Proof. Assume that $a(s), s \in[0,1]$, is a continuous family of unbalanced weights of $\mathbb{A}$. Then $\mu(\mathbb{A}, a(s))$ does not depend on $s$. Indeed, using equations (4.1) one shows that the critical points cannot approach $\pi^{-1}(D)$ as $s \in[0,1]$ changes. For generic weights $a$ we have $\mu(\mathbb{A}, a(s))=|\chi(U)|$ by Theorem 2.4. Hence, $\mu(\mathbb{A}, a)=|\chi(U)|$ for any unbalanced weights $a$.

\section{Hamiltonians of good fibers}

\subsection{Construction}

Consider the master function

$$
\Phi(z, t)=\sum_{j \in J} a_{j} \log f_{j}(z, t)
$$

as a function on $\tilde{U} \subset \mathbb{C}^{n} \times \mathbb{C}^{k}$.

Let $\kappa$ be a nonzero complex number. The function $e^{\Phi(z, t) / \kappa}$ defines a rank one local system $\mathcal{L}_{\kappa}$ on $\tilde{U}$ whose horizontal sections over open subsets of $\tilde{U}$ are univalued branches of $e^{\Phi(z, t) / \kappa}$ multiplied by complex numbers.

For a fixed $z$, choose any $\gamma \in H_{k}\left(U(\mathbb{A}(z)),\left.\mathcal{L}_{\kappa}\right|_{U(\mathbb{A}(z))}\right)$. The linear map

$$
\{\gamma\}: \mathcal{A}^{k}(\mathbb{A}(z)) \rightarrow \mathbb{C}, \quad \omega \mapsto \int_{\gamma} e^{\Phi(z, t) / \kappa} \omega,
$$

is an element of $\operatorname{Sing} \mathcal{F}^{k}(\mathbb{A}(z))$ by Stokes' theorem.

It is known that for generic $\kappa$ any element of $\operatorname{Sing} \mathcal{F}^{k}(\mathbb{A}(z))$ corresponds to a certain $\gamma$ and in that case the integration identifies $\operatorname{Sing} \mathcal{F}^{k}(\mathbb{A}(z))$ and $H_{k}\left(U(\mathbb{A}(z)),\left.\mathcal{L}_{\kappa}\right|_{U(\mathbb{A}(z))}\right)$, see [23].

The vector bundle

$$
\cup_{z \in \mathbb{C}^{n}-\Delta} H_{k}\left(U(\mathbb{A}(z)),\left.\mathcal{L}_{\kappa}\right|_{U(\mathbb{A}(z))}\right) \rightarrow \mathbb{C}^{n}-\Delta
$$


has a canonical (flat) Gauss-Manin connection. The Gauss-Manin connection induces a flat connection on the trivial bundle $\mathbb{C}^{n} \times \operatorname{Sing} V \rightarrow \mathbb{C}^{n}$ with singularities over the discriminant $\Delta \subset \mathbb{C}^{n}$. That connection will be called the Gauss-Manin connection as well.

Theorem 5.1. The Gauss-Manin differential equations for horizontal sections of the connection on $\mathbb{C}^{n} \times \operatorname{Sing} V \rightarrow \mathbb{C}^{n}$ have the form

$$
\kappa \frac{\partial I}{\partial z_{j}}(z)=K_{j}(z) I(z), \quad j \in J
$$

where $I(z) \in \operatorname{Sing} V$ is a horizontal section, $K_{j}(z): V \rightarrow V, j \in J$, are suitable linear operators preserving $\operatorname{Sing} V$ and independent on $\kappa$. For every $j$, the operator $K_{j}(z)$ is a rational function of $z$ regular on $\mathbb{C}^{n}-\Delta$. Each operator is symmetric with respect to the contravariant form $S^{(a)}$.

Theorem 5.1 is proved in Section 5.3. A formula for $K_{j}(z)$ see in (5.3).

The flatness of the connection for all $\kappa$ implies the commutativity of the operators,

$$
\left.\left.K_{i}(z)\right|_{\operatorname{Sing} V} K_{j}(z)\right|_{\operatorname{Sing} V}=\left.\left.K_{j}(z)\right|_{\operatorname{Sing} V} K_{i}(z)\right|_{\operatorname{Sing} V} \quad \text { for all } i, j \text { and } z \in \mathbb{C}^{n}-\Delta .
$$

Let $V^{*}$ be the space dual to $V$. If $M: V \rightarrow V$ is a linear operator, then $M^{*}: V^{*} \rightarrow V^{*}$ denotes the dual operator. Let $W \subset V^{*}$ be the image of $V$ under the map $V \rightarrow V^{*}$ associated with the contravariant form and $\operatorname{Sing} W \subset W$ the image of $\operatorname{Sing} V$. The contravariant form induces on $W$ a nondegenerate symmetric bilinear form, also denoted by $S^{(a)}$.

Lemma 5.2. For $z \in \mathbb{C}^{n}-\Delta$, the operators $K_{j}(z)^{*}$ preserve the subspaces $\operatorname{Sing} W \subset W \subset V^{*}$. The operators $\left.K_{j}(z)^{*}\right|_{W}: W \rightarrow W$ are symmetric with respect to the contravariant form. The operators $\left.K_{j}(z)^{*}\right|_{\operatorname{Sing} W}: \operatorname{Sing} W \rightarrow \operatorname{Sing} W, j \in J$, commute.

For $z \in \mathbb{C}^{n}-\Delta$, we define the quantum integrable model assigned to $(\mathbb{A}(z), a)$ to be the collection

$$
\left(\operatorname{Sing} W ;\left.S^{(a)}\right|_{\operatorname{Sing} W} ;\left.K_{1}(z)^{*}\right|_{\operatorname{Sing} W}, \ldots,\left.K_{n}(z)^{*}\right|_{\operatorname{Sing} W}: \operatorname{Sing} W \rightarrow \operatorname{Sing} W\right) .
$$

The unital subalgebra of End(Sing $W$ ) generated by operators $\left.K_{1}(z)^{*}\right|_{\operatorname{Sing} W}, \ldots,\left.K_{n}(z)^{*}\right|_{\operatorname{Sing} W}$ will be called the algebra of geometric Hamiltonians of $(\mathbb{A}(z), a)$.

If the contravariant form $S^{(a)}$ is nondegenerate on $V$, then this model is isomorphic to the collection

$$
\left(\operatorname{Sing} V ;\left.S^{(a)}\right|_{\operatorname{Sing} V} ;\left.K_{1}(z)\right|_{\operatorname{Sing} V}, \ldots,\left.K_{n}(z)\right|_{\operatorname{Sing} V}: \operatorname{Sing} V \rightarrow \operatorname{Sing} V\right) .
$$

It is clear that any weighted essential arrangement with normal crossings only can be realized as a good fiber of such a construction. Thus, every weighted essential arrangement with normal crossings only is provided with a quantum integrable model.

\section{$5.2 \quad$ Key identity $(5.2)$}

For any circuit $C=\left\{i_{1}, \ldots, i_{r}\right\} \subset J$, let us define a linear operator $L_{C}: V \rightarrow V$ in terms of the standard basis of $V$, see Section 2.11.

For $m=1, \ldots, r$, define $C_{m}=C-\left\{i_{m}\right\}$. Let $\left\{j_{1}<\cdots<j_{k}\right\} \subset J$ be an independent ordered subset and $F\left(H_{j_{1}}, \ldots, H_{j_{k}}\right)$ the corresponding element of the standard basis. Define $L_{C}: F\left(H_{j_{1}}, \ldots, H_{j_{k}}\right) \mapsto 0$ if $\left|\left\{j_{1}, \ldots, j_{k}\right\} \cap C\right|<r-1$. If $\left\{j_{1}, \ldots, j_{k}\right\} \cap C=C_{m}$ for some $1 \leqslant m \leqslant r$, then using the skew-symmetry property (2.5) we can write

$$
F\left(H_{j_{1}}, \ldots, H_{j_{k}}\right)= \pm F\left(H_{i_{1}}, H_{i_{2}}, \ldots, \widehat{H_{i_{m}}}, \ldots, H_{i_{r-1}} H_{i_{r}}, H_{s_{1}}, \ldots, H_{s_{k-r+1}}\right)
$$


with $\left\{s_{1}, \ldots, s_{k-r+1}\right\}=\left\{j_{1}, \ldots, j_{k}\right\}-C_{m}$. Define

$$
\begin{aligned}
L_{C}: & F\left(H_{i_{1}}, \ldots, \widehat{H_{i_{m}}}, \ldots, H_{i_{r}}, H_{s_{1}}, \ldots, H_{s_{k-r+1}}\right) \\
& \mapsto(-1)^{m} \sum_{l=1}^{r}(-1)^{l} a_{i_{l}} F\left(H_{i_{1}}, \ldots, \widehat{H_{i_{l}}}, \ldots, H_{i_{r}}, H_{s_{1}}, \ldots, H_{s_{k-r+1}}\right) .
\end{aligned}
$$

Lemma 5.3. The map $L_{C}$ is symmetric with respect to the contravariant form.

Proof. For $l=1, \ldots, r$, denote $F_{l}=F\left(H_{i_{1}}, \ldots, \widehat{H_{i_{l}}}, \ldots, H_{i_{r}}, H_{s_{1}}, \ldots, H_{s_{k-r+1}}\right)$. It is clear that $L_{C}$ is symmetric with respect to the contravariant form if and only if $S^{(a)}\left(L_{C} F_{l}, F_{m}\right)=$ $S^{(a)}\left(F_{l}, L_{C} F_{m}\right)$ for all $1 \leqslant l, m \leqslant r$. But both sides of this expression are equal to $(-1)^{l+m} a_{i_{1}} \cdots a_{i_{r}} a_{s_{1}} \cdots a_{s_{k-r+1}}$.

On $\mathbb{C}^{n} \times \mathbb{C}^{k}$ consider the logarithmic differential 1-forms

$$
\omega_{j}=\frac{d f_{j}}{f_{j}}, j \in J, \quad \omega_{C}=\frac{d f_{C}}{f_{C}}, \quad C \in \mathfrak{C} .
$$

For any circuit $C=\left\{i_{1}, \ldots, i_{r}\right\}$, we have

$$
\omega_{i_{1}} \wedge \cdots \wedge \omega_{i_{r}}=\omega_{C} \wedge \sum_{l=1}^{r}(-1)^{l-1} \omega_{i_{1}} \wedge \cdots \wedge \widehat{\omega_{i_{l}}} \wedge \cdots \wedge \omega_{i_{r}} .
$$

Lemma 5.4. We have

$$
\begin{aligned}
& \sum_{\substack{\text { independent } \\
\left\{j_{1}<\cdots<j_{k}\right\} \subset J}}\left(\sum_{j \in J} a_{j} \omega_{j}\right) \wedge \omega_{j_{1}} \wedge \cdots \wedge \omega_{j_{k}} \otimes F\left(H_{j_{1}}, \ldots, H_{j_{k}}\right) \\
& =\sum_{\substack{\text { independent } \\
\left\{j_{1}<\cdots<j_{k}\right\} \subset J}} \sum_{C \in \mathfrak{C}} \omega_{C} \wedge \omega_{j_{1}} \wedge \cdots \wedge \omega_{j_{k}} \otimes L_{C} F\left(H_{j_{1}}, \ldots, H_{j_{k}}\right) .
\end{aligned}
$$

Proof. The lemma is a direct corollary of the definition of maps $L_{C}$.

Identity (5.2) is a key formula of this paper. Identity (5.2) is an analog of the key Theorem 7.2.5 in [23] and it is a generalization of the identity of Lemma 4.2 in [27].

\subsection{An application of the key identity (5.2) - proof of Theorem 5.1}

Fix $\kappa \in \mathbb{C}^{\times}, z \in \mathbb{C}^{n}-\Delta, \gamma \in H_{k}\left(U(\mathbb{A}(z)),\left.\mathcal{L}_{\kappa}\right|_{U(\mathbb{A}(z))}\right)$. Let $\{\gamma\}: \mathcal{A}^{k}(\mathbb{A}(z)) \rightarrow \mathbb{C}, \omega \mapsto$ $\int_{\gamma} e^{\Phi(z, t) / \kappa} \omega$, be the corresponding element of $V$. We have

$$
\{\gamma\}=\sum_{\substack{\text { independent } \\\left\{j_{1}<\cdots<j_{k}\right\} \subset J}}\left(\int_{\gamma} e^{\Phi(z, t) / \kappa} \omega_{j_{1}} \wedge \cdots \wedge \omega_{j_{k}}\right) F\left(H_{j_{1}}, \ldots, H_{j_{k}}\right) .
$$

Let $z \mapsto \gamma(z) \in H_{k}\left(U(\mathbb{A}(z)),\left.\mathcal{L}_{\kappa}\right|_{U(\mathbb{A}(z))}\right)$ be a locally constant section of the Gauss-Manin connection. Then

$$
\{\gamma(z)\}=\sum_{\substack{\text { independent } \\\left\{j_{1}<\cdots<j_{k}\right\} \subset J}}\left(\int_{\gamma(z)} e^{\Phi(z, t) / \kappa} \omega_{j_{1}} \wedge \cdots \wedge \omega_{j_{k}}\right) F\left(H_{j_{1}}, \ldots, H_{j_{k}}\right) .
$$


Lemma 5.5. The differential of the function $\{\gamma(z)\}$ is given by the formula

$$
\kappa d\{\gamma(z)\}=\sum_{C \in \mathfrak{C}} L_{C}\{\gamma(z)\} \omega_{C}
$$

Proof. The lemma follows from identity (5.2) and the formula of differentiation of an integral.

Lemma 5.6. For every circuit $C$, the operator $L_{C}$ preserves the subspace $\operatorname{Sing} V$.

Proof. The values of the function $\{\gamma(z)\}$ belong to $\operatorname{Sing} V$. Hence, the values of its derivatives belong to $\operatorname{Sing} V$. Now the lemma follows from Lemma 5.5.

Recall that $\omega_{C}=d f_{C} / f_{C}$ and $f_{C}=\sum_{j \in J} \lambda_{j}^{C} z_{j}$. Denote

$$
K_{j}(z)=\sum_{C \in \mathfrak{C}} \frac{\lambda_{j}^{C}}{f_{C}(z)} L_{C}, \quad j \in J
$$

Then

$$
\sum_{C \in \mathfrak{C}} \omega_{C} \otimes L_{C}=\sum_{j \in J} d z_{j} \otimes K_{j}(z)
$$

Lemma 5.7. Let $z \mapsto \gamma(z) \in H_{k}\left(U(\mathbb{A}(z)),\left.\mathcal{L}_{\kappa}\right|_{U(\mathbb{A}(z))}\right)$ be a locally constant section of the GaussManin connection. Then

$$
\kappa \frac{\partial}{\partial z_{j}}\{\gamma(z)\}=K_{j}(z)\{\gamma(z)\}, \quad j \in J
$$

Lemmas 5.3, 5.6, 5.7 prove Theorem 5.1.

\subsection{Another application of the key identity (5.2)}

Recall that $\tilde{U}$ is the complement to the union of hyperplanes $\left(\tilde{H}_{j}\right)_{j \in J}$ in $\mathbb{C}^{n} \times \mathbb{C}^{k}$, see Section 3.1. Denote by $\mathbb{C}(z, t)_{\tilde{U}}$ the algebra of rational functions on $\mathbb{C}^{n} \times \mathbb{C}^{k}$ regular on $\tilde{U}$.

For any basis vector $\left(H_{j_{1}}, \ldots, H_{j_{k}}\right)$ of $V^{*}$, let us write

$$
\omega_{j_{1}} \wedge \cdots \wedge \omega_{j_{k}}=f_{j_{1}, \ldots, j_{k}}(z, t) d t_{1} \wedge \cdots \wedge d t_{k}+z \text {-part }
$$

where $f_{j_{1}, \ldots, j_{k}} \in \mathbb{C}(z, t)_{\tilde{U}}$ and the $z$-part is a differential form with zero restriction to any fiber of the projection $\mathbb{C}^{n} \times \mathbb{C}^{k} \rightarrow \mathbb{C}^{n}$ (in coordinates $t_{1}, \ldots, t_{k}, z_{1}, \ldots, z_{n}$, that form has at least one of $d z_{1}, \ldots, d z_{n}$ as factors in each of its summands). Define the canonical element $\tilde{E} \in \mathbb{C}(z, t)_{\tilde{U}} \otimes V$ by the condition

$$
\left\langle\tilde{E}, 1 \otimes\left(H_{j_{1}}, \ldots, H_{j_{k}}\right)\right\rangle=f_{j_{1}, \ldots, j_{k}},
$$

for any independent $\left\{j_{1}, \ldots, j_{k}\right\} \subset J$.

Theorem 5.8. For any $j \in J$, there exist elements $h_{1}, \ldots, h_{k} \in \mathbb{C}(z, t)_{\tilde{U}} \otimes V$ such that

$$
\left(1 \otimes K_{j}(z)\right) \tilde{E}(z, t)=\left(\frac{a_{j}}{f_{j}(z, t)} \otimes 1\right) \tilde{E}(z, t)+\sum_{i=1}^{k}\left(\frac{\partial \Phi}{\partial t_{i}}(z, t) \otimes 1\right) h_{i}(z, t) .
$$


Proof. We have

$$
\nu(a)=\sum_{i=1}^{k} \frac{\partial \Phi}{\partial t_{i}}(z, t) d t_{i}+\sum_{j \in J} \frac{a_{j}}{f_{j}(z, t)} d z_{j}
$$

and

$$
\sum_{\substack{\text { independent } \\\left\{j_{1}<\cdots<j_{k}\right\} \subset J}} \omega_{j_{1}} \wedge \cdots \wedge \omega_{j_{k}} \otimes F\left(H_{j_{1}}, \ldots, H_{j_{k}}\right)=\tilde{E}(z, t)\left(d t_{1} \wedge \cdots \wedge d t_{k} \otimes 1\right)+z \text {-part }
$$

where $z$-part is a $V$-valued differential $k$-form with zero restriction to each fiber of the projection $\mathbb{C}^{n} \times \mathbb{C}^{k} \rightarrow \mathbb{C}^{n}$. Then identity (5.2) and formulas (5.6), (5.7), (5.4) imply the theorem.

\subsection{Hamiltonians, critical points and the canonical element}

Fix $z \in \mathbb{C}^{n}-\Delta$. Recall that in Section 5.1 we have defined the quantum integrable model assigned to $(\mathbb{A}(z), a)$ to be the collection

$$
\left(\operatorname{Sing} W ;\left.S^{(a)}\right|_{\operatorname{Sing} W} ;\left.K_{1}(z)^{*}\right|_{\operatorname{Sing} W}, \ldots,\left.K_{n}(z)^{*}\right|_{\operatorname{Sing} W}: \operatorname{Sing} W \rightarrow \operatorname{Sing} W\right) .
$$

Let $p \in U(\mathbb{A}(z))$ be an isolated critical point of the master function $\Phi(z, \cdot): U(\mathbb{A}(z)) \rightarrow \mathbb{C}$. Let $A_{p, \Phi}$ be the local algebra of the critical point and [ ]: $\mathbb{C}(t)_{U(\mathbb{A}(z))} \rightarrow A_{p, \Phi}$ the canonical projection. Denote by $\operatorname{Hess}^{(\mathrm{a})}$ the Hessian of $\Phi(z, \cdot)$ with respect to variables $t_{1}, \ldots, t_{k}$.

Let $E \in \mathbb{C}(t)_{U(\mathbb{A}(z))} \otimes V$ be the canonical element associated with $\mathbb{A}(z)$, see Section 2.10. Denote by $[E]$ its projection to $A_{p, \Phi} \otimes V$. By Lemma 2.6 we have $[E] \in A_{p, \Phi} \otimes \operatorname{Sing} V$. Let $V \rightarrow W$ be the map associate with the contravariant form and $[\boldsymbol{E}]$ the image of $[E]$ under the induced map $A_{p, \Phi} \otimes \operatorname{Sing} V \rightarrow A_{p, \Phi} \otimes \operatorname{Sing} W$,

$$
[\boldsymbol{E}] \in A_{p, \Phi} \otimes \operatorname{Sing} W
$$

Theorem 5.9. We have

(i) $S^{(a)}([\boldsymbol{E}],[\boldsymbol{E}])=(-1)^{k}\left[\operatorname{Hess}^{(a)}\right]$,

(ii) $\left(1 \otimes K_{j}(z)^{*}\right)[\boldsymbol{E}]=\left(\left[a_{j} / f_{j}(z, \cdot)\right] \otimes 1\right)[\boldsymbol{E}]$ for $j \in J$.

Proof. Part $(i)$ follows from Theorem 2.8. Part $(i i)$ follows from Theorem 5.8.

Remark 5.10. The elements $\left[a_{j} / f_{j}(z, \cdot)\right], j \in J$, generate $A_{p, \Phi}$ due to Lemma 2.5 and the assumption $\left(a_{j} \neq 0\right.$ for all $\left.j\right)$.

\section{Asymptotic solutions and eigenvectors of Hamiltonians}

\subsection{Asymptotic solutions, one variable}

Let $u$ be a variable, $W$ a vector space, $M(u) \in \operatorname{End}(W)$ an endomorphism depending holomorphically on $u$ at $u=0$. Consider a differential equation,

$$
\kappa \frac{d I}{d u}(u)=M(u) I(u)
$$

depending on a complex parameter $\kappa \in \mathbb{C}^{*}$. 
Let $P(u) \in \mathbb{C},\left(w_{m}(u) \in W\right)_{m \in \mathbb{Z}_{\geqslant 0}}$ be functions holomorphic at $u=0$ and $w_{0}(0) \neq 0$. The series

$$
I(u, \kappa)=e^{P(u) / \kappa} \sum_{m=0}^{\infty} w_{m}(u) \kappa^{m}
$$

will be called an asymptotic solution to (6.1) if it satisfies (6.1) to all orders in $\kappa$. In particular, the leading order equation is

$$
\frac{d P}{d u}(u) w_{0}(u)=M(u) w_{0}(u)
$$

Assume now that

$$
M(u)=\frac{M_{-1}}{u}+M_{0}+M_{1} u+\cdots, \quad M_{j} \in \operatorname{End}(W),
$$

has a first order pole at $u=0$ and $I(u, \kappa)$ is a series like in $(6.2)$. The series $I(u, \kappa)$ will be called an asymptotic solution to equation (6.1) with such $M(u)$ if it satisfies (6.1) to all orders in $\kappa$. In particular, the leading order equation is again equation (6.3). Equation (6.3) implies

$$
w_{0}(0) \in \operatorname{ker} M_{-1}, \quad \frac{d P}{d u}(0) w_{0}(0)=M_{0} w_{0}(0)+M_{-1} \frac{d w_{0}}{d u}(0) .
$$

\subsection{Critical points of the master function and asymptotic solutions}

Let us return to the situation of Section 3.

Let $t(z)$ be a nondegenerate critical point of $\Phi(z, \cdot): U(\mathbb{A}(z)) \rightarrow \mathbb{C}$. Assume that $t(z)$ depends on $z$ holomorphically in a neighborhood of a point $z^{0} \in \mathbb{C}^{n}$. Fix a univalued branch of $\Phi$ in a neighborhood of $\left(z^{0}, t\left(z^{0}\right)\right)$ (by choosing arguments of all of the logarithms). Denote $\Psi(z)=\Phi(z, t(z))$.

Let $B \subset \mathbb{C}^{k}$ be a small ball with center at $t\left(z^{0}\right)$. Denote

$$
B^{-}=\left\{t \in B \mid \operatorname{Re} \Phi\left(z^{0}, t\left(z^{0}\right)\right)>\operatorname{Re} \Phi\left(z^{0}, t\right)\right\} .
$$

It is well known that $H_{k}\left(B, B^{-} ; \mathbb{Z}\right)=\mathbb{Z}$, see for example [1]. There exist local coordinates $u_{1}, \ldots, u_{k}$ on $\mathbb{C}^{k}$ centered at $t\left(z^{0}\right)$ such that $\Phi\left(z^{0}, u\right)=-u_{1}^{2}-\cdots-u_{k}^{2}+$ const. Denote

$$
\delta=\left\{\left(u_{1}, \ldots, u_{k}\right) \in \mathbb{R}^{k} \mid u_{1}^{2}+\cdots+u_{k}^{2} \leqslant \epsilon\right\}
$$

where $\epsilon$ is a small positive number. That $\delta$, considered as a $k$-chain, generates $H_{k}\left(B, B^{-} ; \mathbb{Z}\right)$.

Define an element $\{\delta\}(z, \kappa) \in V$ by the formula

$$
\{\delta\}(z, \kappa): V^{*} \rightarrow \mathbb{C}, \quad \omega \mapsto \kappa^{-k / 2} \int_{\delta} e^{\Phi(z, t) / \kappa} \omega(z, t) .
$$

Recall that any element $\omega \in V^{*}$ is a linear combination of elements $\left(H_{j_{1}}, \ldots, H_{j_{k}}\right)$ and such an element $\left(H_{j_{1}}, \ldots, H_{j_{k}}\right)$ is identified with the differential form

$$
\omega_{j_{1}} \wedge \cdots \wedge \omega_{j_{k}}=d f_{j_{1}}(z, t) / f_{j_{1}}(z, t) \wedge \cdots \wedge d f_{j_{k}}(z, t) / f_{j_{k}}(z, t) .
$$

In (6.5) we integrate over $\delta$ such a differential form multiplied by $e^{\Phi(z, t) / \kappa}$.

The element $\{\delta\}(z, \kappa)$ as a function of $z, \kappa$ is holomorphic if $z$ is close to $z^{0}$ and $\kappa \neq 0$. 


\section{Theorem 6.1.}

(i) Let $\kappa \in \mathbb{R}$ and $\kappa \rightarrow+0$. Then the function $\{\delta\}(z, \kappa)$ has an asymptotic expansion

$$
\{\delta\}(z, \kappa)=e^{\Psi(z) / \kappa} \sum_{m=0}^{\infty} w_{m}(z) \kappa^{m},
$$

where $\left(w_{m}(z) \in V\right)_{m \in \mathbb{Z}_{\geqslant 0}}$ are functions of $z$ holomorphic at $z^{0}$ and

$$
w_{0}(z)= \pm(2 \pi)^{k / 2}\left((-1)^{k} \operatorname{det}_{1 \leqslant i, j \leqslant k}\left(\frac{\partial^{2} \Phi}{\partial t_{i} \partial t_{j}}\right)(z, t(z))\right)^{-1 / 2} v(z, t(z)) .
$$

Here $v(z, t(z))$ is the special vector associated with the critical point $(z, t(z))$ of the function $\Phi(z, \cdot)$, see Section 2.10. The sign \pm depends on the choice of the orientation of $\delta$.

(ii) The asymptotic expansion (6.6) gives an asymptotic solution to the Gauss-Manin differential equations (5.5).

(iii) The functions $\left(w_{m}(z)\right)_{m \in \mathbb{Z}_{\geqslant 0}}$ take values in $\operatorname{Sing} V$.

Part $(i)$ of the theorem is a direct corollary of the method of steepest descent; see, for example, $\S 11$ in [1]. Part (ii) follows from Lemma 5.4 and formula of differentiation of an integral. Part (iii) follows from Stokes' theorem.

Remark 6.2. The definition of $\delta$ depends on the choice of local coordinates $u_{1}, \ldots, u_{k}$, but the asymptotic expansion (6.6) does not depend on the choice of $\delta$ since the difference of the corresponding integrals is exponentially small.

Corollary 6.3. Let $z^{0}, t=t(z), \Psi(z)$ be the same as in Theorem 6.1. Assume that $z^{0} \in \mathbb{C}^{n}-\Delta$. In that case the operators $\left.K_{j}\left(z^{0}\right)\right|_{\operatorname{Sing} V}: \operatorname{Sing} V \rightarrow \operatorname{Sing} V, j \in J$, are all well-defined, see (5.3), and we have

$$
K_{j}\left(z^{0}\right) v\left(z^{0}, t\left(z^{0}\right)\right)=\frac{\partial \Psi}{d z_{j}}\left(z^{0}\right) v\left(z^{0}, t\left(z^{0}\right)\right), \quad j \in J,
$$

Thus, the special vector $v\left(z^{0}, t\left(z^{0}\right)\right)$ is an eigenvector of the geometric Hamiltonians $K_{j}\left(z^{0}\right)$.

Proof. The corollary follows from equation (6.3).

Note that $\frac{\partial \Psi}{d z_{j}}\left(z^{0}\right)=\frac{a_{j}}{f_{j}\left(z^{0}, t\left(z^{0}\right)\right)}$.

Remark 6.4. The Gauss-Manin differential equations (5.5) have singularities over the discriminant $\Delta \subset \mathbb{C}^{n}$. If $z^{0} \in \Delta$, then expansion (6.6) still gives an asymptotic solution to equations (5.5) and that asymptotic solution is regular at $z^{0}$.

\section{Hamiltonians of bad fibers}

\subsection{Naive geometric Hamiltonians}

Let us return to the situation of Section 3. Let $z^{0} \in \Delta$ and $z \in \mathbb{C}^{n}-\Delta$. We have

$$
\begin{aligned}
& \operatorname{Sing} \mathcal{F}^{k}\left(\mathbb{A}\left(z^{0}\right)\right) \subset \operatorname{Sing} \mathcal{F}^{k}(\mathbb{A}(z)) \subset \mathcal{F}^{k}(\mathbb{A}(z)), \\
& \operatorname{Sing} \mathcal{F}^{k}\left(\mathbb{A}\left(z^{0}\right)\right) \subset \mathcal{F}^{k}\left(\mathbb{A}\left(z^{0}\right)\right) \subset \mathcal{F}^{k}(\mathbb{A}(z)), \\
& \operatorname{Sing} \mathcal{F}^{k}\left(\mathbb{A}\left(z^{0}\right)\right)=\mathcal{F}^{k}\left(\mathbb{A}\left(z^{0}\right)\right) \cap\left(\operatorname{Sing} \mathcal{F}^{k}(\mathbb{A}(z))\right),
\end{aligned}
$$

see Section 3.4. Recall that $\mathcal{F}^{k}(\mathbb{A}(z))$ was denoted by $V$. 
Consider the map $V \rightarrow V^{*}$ corresponding to the contravariant form. In Section 5.1 we denoted the images of $V$ and $\operatorname{Sing} V$ by $W$ and $\operatorname{Sing} W$, respectively. We denote the images of $\mathcal{F}^{k}\left(\mathbb{A}\left(z^{0}\right)\right)$ and Sing $\mathcal{F}^{k}\left(\mathbb{A}\left(z^{0}\right)\right)$ by $W\left(z^{0}\right)$ and Sing $W\left(z^{0}\right)$, respectively. We have

Sing $W\left(z^{0}\right) \subset \operatorname{Sing} W \subset W, \quad$ Sing $W\left(z^{0}\right) \subset W\left(z^{0}\right) \subset W$.

Recall that $\Delta$ is the union of hyperplanes $H_{C}, C \in \mathfrak{C}$. Denote

$$
\mathfrak{C}_{0}=\left\{C \in \mathfrak{C} \mid z^{0} \in H_{C}\right\} .
$$

Consider the operator-valued functions $K_{j}(z): V \rightarrow V, j \in J$, given by formula (5.3). Denote

$$
K_{j}^{0}(z)=\sum_{C \in \mathfrak{C}_{0}} \frac{\lambda_{j}^{C}}{f_{C}(z)} L_{C}, \quad K_{j}^{1}(z)=K_{j}(z)-K_{j}^{0}(z) .
$$

Each of the summands of $K_{j}^{0}(z)$ tends to infinity as $z$ tends to $z^{0}$ in $\mathbb{C}^{n}-\Delta$. The operator-valued function $K_{j}^{1}(z)$ is regular at $z^{0}$.

The operators $K_{j}(z)^{*}, L_{C}^{*}$ preserve the subspaces Sing $W \subset W \subset V^{*}$ and are symmetric operators on $W$ with respect to the contravariant form on $W$. The operators $K_{j}(z)^{*}$ restricted to Sing $W$ commute.

The point $z^{0} \in \Delta$ defines an edge $X_{z^{0}}$ of the arrangement $\left(H_{C}\right)_{C \in \mathfrak{C}}$, where $X_{z^{0}}=\cap_{C \in \mathfrak{C}_{0}} H_{C}$. Denote by $T_{z^{0}}$ the vector space of constant vectors fields on $\mathbb{C}^{n}$ which are tangent to $X_{z^{0}}$,

$$
T_{z^{0}}=\left\{\xi=\sum_{j \in J} \xi_{j} \frac{\partial}{\partial z_{j}} \mid \xi_{j} \in \mathbb{C}, \xi\left(f_{C}\right)=0 \text { for all } C \in \mathfrak{C}_{0}\right\} .
$$

Lemma 7.1. For any $\xi \in T_{z^{0}}$,

(i) The linear operator

$$
K_{\xi}(z)=\sum_{j \in J} \xi_{j} K_{j}(z): V \rightarrow V
$$

considered as a function of $z$, is regular at $z^{0}$, moreover,

$$
K_{\xi}(z)=\sum_{j \in J} \xi_{j} K_{j}^{1}(z)
$$

(ii) The linear operator $K_{\xi}\left(z^{0}\right)$ preserves the subspace $\mathcal{F}^{k}\left(\mathbb{A}\left(z^{0}\right)\right) \subset V$.

(iii) The dual linear operator

$$
K_{\xi}(z)^{*}: V^{*} \rightarrow V^{*}
$$

considered as a function of $z$, is regular at $z^{0}$, moreover,

$$
K_{\xi}(z)^{*}=\sum_{j \in J} \xi_{j} K_{j}^{1}(z)^{*}
$$

(iv) The linear operator $K_{\xi}\left(z^{0}\right)^{*}$ preserves the subspace $\operatorname{Sing} W\left(z^{0}\right) \subset V^{*}$.

Proof. Parts $(i i i),(i v)$ follow from parts $(i),(i i)$. Part $(i)$ is clear. Part $(i i)$ follows from a straightforward calculation.

The operators $K_{\xi}\left(z^{0}\right)^{*}$ preserve the subspace Sing $W\left(z^{0}\right)$. The operators

$$
\left.K_{\xi}\left(z^{0}\right)^{*}\right|_{\operatorname{Sing} W\left(z^{0}\right)}: \operatorname{Sing} W\left(z^{0}\right) \rightarrow \operatorname{Sing} W\left(z^{0}\right), \quad \xi \in T_{z^{0}}
$$

form a commutative family of linear operators. The operators are symmetric with respect to the contravariant form. These operators will be called naive geometric Hamiltonians on Sing $W\left(z^{0}\right)$. 


\subsection{Space $\mathcal{F}^{k}\left(\mathbb{A}\left(z^{0}\right)\right)$ and operators $L_{C}$}

\section{Lemma 7.2.}

(i) The space $\mathcal{F}^{k}\left(\mathbb{A}\left(z^{0}\right)\right)$ lies in the kernel of $L_{C}: V \rightarrow V$ for any $C \in \mathfrak{C}_{0}$.

(ii) The space $W\left(z^{0}\right)$ lies in the kernel of $\left.L_{C}^{*}\right|_{W}: W \rightarrow W$ for any $C \in \mathfrak{C}_{0}$.

(iii) For any $C \in \mathfrak{C}_{0}$, the image of $\left.L_{C}^{*}\right|_{W}$ is orthogonal to $W\left(z^{0}\right)$ with respect to the contravariant form.

Proof. Part ( $i$ ) follows from a straightforward easy calculation. Part (ii) follows from part $(i)$. Part (iii) follows from part $(i i)$ and the fact that $L_{C}^{*}$ is symmetric.

\subsection{Conjecture}

Conjecture 7.3. Let $z^{0} \in \Delta$. Assume that the contravariant form restricted to $\operatorname{Sing} W\left(z^{0}\right)$ is nondegenerate. Let $\mathrm{pr}: \operatorname{Sing} W \rightarrow \operatorname{Sing} W\left(z^{0}\right)$ be the orthogonal projection with respect to the contravariant form. Then the linear operators

$$
\left.\operatorname{pr} K_{j}^{1}\left(z^{0}\right)^{*}\right|_{\operatorname{Sing} W\left(z^{0}\right)}: \operatorname{Sing} W\left(z^{0}\right) \rightarrow \operatorname{Sing} W\left(z^{0}\right), \quad j \in J,
$$

commute and are symmetric with respect to the contravariant form.

For $z^{0} \in \Delta$, we define the quantum integrable model assigned to $\left(\mathbb{A}\left(z^{0}\right), a\right)$ to be the collection

$$
\begin{aligned}
& \left(\operatorname{Sing} W\left(z^{0}\right),\left.S^{(a)}\right|_{\operatorname{Sing} W\left(z^{0}\right)},\right. \\
& \left.\left.\quad \operatorname{pr} K_{j}^{1}\left(z^{0}\right)^{*}\right|_{\operatorname{Sing} W\left(z^{0}\right)}: \operatorname{Sing} W\left(z^{0}\right) \rightarrow \operatorname{Sing} W\left(z^{0}\right), \quad \text { where } j \in J\right) .
\end{aligned}
$$

The unital subalgebra of $\operatorname{End}\left(\operatorname{Sing} W\left(z^{0}\right)\right)$ generated by operators

$$
\left.\operatorname{pr} K_{j}^{1}\left(z^{0}\right)^{*}\right|_{\text {Sing } W\left(z^{0}\right),} \quad j \in J,
$$

will be called the algebra of geometric Hamiltonians of $\left(\mathbb{A}\left(z^{0}\right), a\right)$.

Note that the naive geometric Hamiltonians are elements of the algebra of geometric Hamiltonians, since for any $\xi=\sum_{j \in J} \xi_{j} \frac{\partial}{\partial z_{j}} \in T_{X_{z^{0}}}$, we have

$$
\left.K_{\xi}\left(z^{0}\right)^{*}\right|_{\operatorname{Sing} W\left(z^{0}\right)}=\left.\sum_{j \in J} \xi_{j} \operatorname{pr} K_{j}^{1}\left(z^{0}\right)^{*}\right|_{\operatorname{Sing} W\left(z^{0}\right)} .
$$

In the next section we prove the conjecture under Assumption 7.4 of certain positivity and reality conditions, see Theorem 7.5. In Section 9 more results in this direction will be obtained, see Theorems 9.16 and 9.17. For applications to the Gaudin model an equivariant version of the conjecture is needed, see Sections 10 and 11.

\subsection{Positive $\left(a_{j}\right)_{j \in J}$, real $\left(g_{j}\right)_{j \in J}$}

Assumption 7.4. Assume that all weights $a_{j}, j \in J$, are positive and all functions $g_{j}=$ $b_{j}^{1} t_{1}+\cdots+b_{j}^{k} t_{k}, j \in J$, have real coefficients $b_{j}^{i}$.

The space $V$ has a real structure, $V=V_{\mathbb{R}} \otimes_{\mathbb{R}} \mathbb{C}$, see Section 2.12. Under Assumption 7.4 all subspaces in (7.1) are real (can be defined by real equations). The contravariant form $S^{(a)}$ is positive definite on $V_{\mathbb{R}}$ and is positive definite on the real parts of all of the subspaces in (7.1).

Denote by pr : Sing $V \rightarrow \operatorname{Sing} \mathcal{F}^{k}\left(\mathbb{A}\left(z^{0}\right)\right)$ the orthogonal projection.

Theorem 7.5. Assume that Assumption 7.4 is satisfied and $z^{0} \in \Delta$. Then the operators

$$
\left.\operatorname{pr} K_{j}^{1}\left(z^{0}\right)\right|_{\operatorname{Sing} \mathcal{F}^{k}\left(\mathbb{A}\left(z^{0}\right)\right)}: \operatorname{Sing} \mathcal{F}^{k}\left(\mathbb{A}\left(z^{0}\right)\right) \rightarrow \operatorname{Sing} \mathcal{F}^{k}\left(\mathbb{A}\left(z^{0}\right)\right), \quad j \in J,
$$

commute and are symmetric with respect to the contravariant form.

Theorem 7.5 proves Conjecture 7.3 under Assumption 7.4. 


\subsection{Proof of Theorem 7.5 for $z^{0} \in \Delta \cap \mathbb{R}^{n}$}

Assume that $z^{0} \in \Delta \cap \mathbb{R}^{n}$. Let $r:(\mathbb{C}, \mathbb{R}, 0) \rightarrow\left(\mathbb{C}^{n}, \mathbb{R}^{n}, z^{0}\right)$ be a germ of a holomorphic curve such that $r(u) \in \mathbb{C}^{n}-\Delta$ for $u \neq 0$. For $u \in \mathbb{R}_{>0}$, the arrangement $\mathbb{A}(r(u))$ is real. Denote $U(r(u))_{\mathbb{R}}=\left(\mathbb{C}^{k}-\cup_{j \in J} H_{j}(r(u))\right) \cap \mathbb{R}^{k}$. Let $U(r(u))_{\mathbb{R}}=\cup_{\alpha} D_{\alpha}(r(u))$ be the decomposition into the union of connected components. We label components so that for any $\alpha$, the component $D_{\alpha}(r(u))$ continuously depends on $u>0$. Let $A$ be the set of all $\alpha$ such that $D_{\alpha}(r(u))$ is bounded. Let $A_{1}$ be the set of all $\alpha$ such that $D_{\alpha}(r(u))$ is bounded and vanishes as $u \rightarrow+0$ (the limit of $D_{\alpha}(r(u))$ is not a domain of $\left.\mathbb{A}\left(z^{0}\right)\right)$. Let $A_{2}$ be the set of all $\alpha$ such that $D_{\alpha}(r(u))$ is bounded and the limit of $D_{\alpha}(r(u))$ as $u \rightarrow 0$ is a domain of $\mathbb{A}\left(z^{0}\right)$. We have $A=A_{1} \cup A_{2}$ and $A_{1} \cap A_{2}=\varnothing$.

All critical points of $\Phi(r(u), \cdot)$ lie in $\cup_{\alpha \in A} D_{\alpha}(r(u))$. Each domain $D_{\alpha}(r(u))$ contains a unique critical point $\left(r(u), t(u)_{\alpha}\right)$ and that critical point is nondegenerate. Denote by $v\left(r(u), t(u)_{\alpha}\right) \in$ Sing $V$ the corresponding special vector. That vector is an eigenvector of the geometric Hamiltonians,

$$
K_{j}(r(u)) v\left(r(u), t(u)_{\alpha}\right)=\frac{a_{j}}{f_{j}\left(r(u), t(u)_{\alpha}\right)} v\left(r(u), t(u)_{\alpha}\right), \quad j \in J .
$$

If $\alpha \in A_{2}$, then all eigenvalues $1 / f_{j}\left(r(u), t(u)_{\alpha}\right), j \in J$, are regular functions at $u=0$. If $\alpha \in A_{1}$, then there is an index $j \in J$ such that $a_{j} / f_{j}\left(r(u), t(u)_{\alpha}\right) \rightarrow \infty$ as $u \rightarrow 0$.

Lemma 7.6. The span $\left\langle v\left(r(u), t(u)_{\alpha}\right)\right\rangle_{\alpha \in A_{2}}$ has a limit as $u \rightarrow+0$. That limit is $\operatorname{Sing} \mathcal{F}^{k}\left(z^{0}\right) \subset$ Sing $V$. Similarly, the span $\left\langle v\left(r(u), t(u)_{\alpha}\right)\right\rangle_{\alpha \in A_{1}}$ has a limit as $u \rightarrow+0$. That limit is (Sing $\left.\mathcal{F}^{k}\left(z^{0}\right)\right)^{\perp} \subset \operatorname{Sing} V$ where ${ }^{\perp}$ denotes the orthogonal complement.

Proof. The lemma follows from Theorem 2.9 and Corollary 2.10.

Assume now that a curve $r(u)=\left(z_{1}(u), \ldots, z_{n}(u)\right)$ is linear in $u$. Then for any $j$ we have $K_{j}^{0}(r(u))=N_{j} / u$ where $N_{j}: \operatorname{Sing} V \rightarrow \operatorname{Sing} V$ is an operator independent of $u$.

Lemma 7.7. The image of $N_{j}$ is a subspace of $\left(\operatorname{Sing} \mathcal{F}^{k}\left(z^{0}\right)\right)^{\perp}$.

Proof. The lemma follows from Theorem 2.9 and Corollary 2.10.

By formula (6.4) and Lemma 7.7, for any $\alpha \in A_{2}$ we have

$$
\frac{a_{j}}{f_{j}\left(r(0), t(0)_{\alpha}\right)} v\left(r(0), t(0)_{\alpha}\right)=K_{j}^{1}(r(0)) v\left(r(0), t(0)_{\alpha}\right)+v_{1}
$$

where $v_{1} \in\left(\operatorname{Sing} \mathcal{F}^{k}\left(z^{0}\right)\right)^{\perp}$. Thus,

$$
\operatorname{pr} K_{j}^{1}(r(0)) v\left(r(0), t(0)_{\alpha}\right)=\frac{1}{f_{j}\left(r(0), t(0)_{\alpha}\right)} v\left(r(0), t(0)_{\alpha}\right) .
$$

Thus, all operators $\operatorname{pr} K_{j}^{1}(r(0))$ are diagonal in the basis $\left(v\left(r(0), t(0)_{\alpha}\right)\right)_{\alpha \in A_{2}}$ of $\operatorname{Sing} \mathcal{F}^{k}\left(\mathbb{A}\left(z^{0}\right)\right)$. Equation (7.2) finishes the proof of the commutativity of operators $\operatorname{pr} K_{j}^{1}\left(z^{0}\right)$.

The symmetry of the operators $\operatorname{pr} K_{j}^{1}\left(z^{0}\right)$ with respect to the contravariant form follows from the fact that operators $\operatorname{pr} K_{j}^{1}\left(z^{0}\right)$ are diagonal in the orthogonal basis $\left(v\left(r(0), t(0)_{\alpha}\right)\right)_{\alpha \in A_{2}}$. 


\subsection{Proof of Theorem 7.5 for any $z^{0} \in \Delta$}

Consider the arrangement $\left(H_{C}\right)_{C \in \mathfrak{C}}$ in $\mathbb{C}^{n}$ and its arbitrary edge $X$. The arrangement $\left(H_{C}\right)_{C \in \mathfrak{C}}$ is real, see Remark 3.2. The edge $X$ is the complexification of $X \cap \mathbb{R}^{n}$.

Denote

$$
\hat{X}=X-\cup_{C \in \mathfrak{C}-\mathfrak{C}_{X}} X \cap H_{C} .
$$

For any $z^{1}, z^{2} \in \hat{X}$, the subspaces

$$
\text { Sing } \mathcal{F}^{k}\left(\mathbb{A}\left(z^{1}\right)\right) \subset \operatorname{Sing} V, \quad \operatorname{Sing} \mathcal{F}^{k}\left(\mathbb{A}\left(z^{2}\right)\right) \subset \operatorname{Sing} V
$$

coincide. Denote that subspace by $\operatorname{Sing} \mathcal{F}^{k}(\mathbb{A}(X)) \subset \operatorname{Sing} V$.

For $z \in \hat{X}$, the operators $\operatorname{pr} K_{j}^{1}(z): \operatorname{Sing} \mathcal{F}^{k}(\mathbb{A}(X)) \rightarrow \operatorname{Sing} \mathcal{F}^{k}(\mathbb{A}(X)), j \in J$, depend on $z$ holomorphically. The operators commute and are symmetric for $z \in \hat{X} \cap \mathbb{R}^{n}$, by reasonings in Section 7.5. Hence they commute and are symmetric for all $z \in \hat{X}$.

\subsection{Critical points and eigenvectors}

Theorem 7.8. Assume Assumption 7.4. Let $z^{0} \in \Delta$ and let $p$ be a critical point of $\Phi\left(z^{0}, \cdot\right)$ : $U\left(\mathbb{A}\left(z^{0}\right)\right) \rightarrow \mathbb{C}$. Then the corresponding special vector $v\left(z^{0}, p\right) \in \operatorname{Sing} \mathcal{F}^{k}\left(z^{0}\right)$ (if nonzero) is an eigenvector of the operators $\operatorname{pr} K_{j}^{1}\left(z^{0}\right), j \in J$,

$$
\operatorname{pr} K_{j}^{1}\left(z^{0}\right) v\left(z^{0}, p\right)=\frac{a_{j}}{f_{j}\left(z^{0}, p\right)} v\left(z^{0}, p\right) .
$$

Proof. If $z^{0} \in \Delta \cap \mathbb{R}^{n}$, then the theorem is just a restatement of formula (7.2).

Assume that $z^{0}$ is an arbitrary point of $\Delta$. Then there exists an edge $X$ of the arrangement $\left(H_{C}\right)_{C \in \mathfrak{C}}$ such that $z^{0} \in \hat{X}$, see (7.3). For $z^{0} \in \hat{X}$, all objects in formula (7.4) depend on $z^{0}$ algebraically. Hence, the fact, that formula (7.4) holds for all critical points if $z^{0} \in \hat{X} \cap \mathbb{R}^{n}$, implies Theorem 7.8 for any $z^{0} \in \hat{X}$.

\subsection{Hamiltonians, critical points and the canonical element}

Let Assumption 7.4 be satisfied. Fix $z^{0} \in \Delta$. We have defined the quantum integrable model assigned to $\left(\mathbb{A}\left(z^{0}\right), a\right)$ to be the collection

$$
\begin{aligned}
& \left(\operatorname{Sing} \mathcal{F}^{k}\left(\mathbb{A}\left(z^{0}\right)\right) ;\left.S^{(a)}\right|_{\operatorname{Sing}} \mathcal{F}^{k}\left(\mathbb{A}\left(z^{0}\right)\right) ;\right. \\
& \left.\left.\quad \operatorname{pr} K_{j}^{1}\left(z^{0}\right)\right|_{\operatorname{Sing} \mathcal{F}^{k}\left(\mathbb{A}\left(z^{0}\right)\right)}: \operatorname{Sing} \mathcal{F}^{k}\left(\mathbb{A}\left(z^{0}\right)\right) \rightarrow \operatorname{Sing} \mathcal{F}^{k}\left(\mathbb{A}\left(z^{0}\right)\right), \text { where } j \in J\right),
\end{aligned}
$$

see Section 7.4.

Let $p \in U\left(\mathbb{A}\left(z^{0}\right)\right)$ be an isolated critical point of the function $\Phi\left(z^{0}, \cdot\right): U\left(\mathbb{A}\left(z^{0}\right)\right) \rightarrow \mathbb{C}$. Let $A_{p, \Phi}$ be the local algebra of the critical point and [ ]: $\mathbb{C}(t)_{U\left(\mathbb{A}\left(z^{0}\right)\right)} \rightarrow A_{p, \Phi}$ the canonical projection. Denote by Hess ${ }^{(a)}$ the Hessian of $\Phi\left(z^{0}, \cdot\right)$.

Let $E \in \mathbb{C}(t)_{U\left(\mathbb{A}\left(z^{0}\right)\right)} \otimes \mathcal{F}^{k}\left(\mathbb{A}\left(z^{0}\right)\right)$ be the canonical element associated with $\mathbb{A}\left(z^{0}\right)$, see Section 2.10. Denote by $[E]$ the projection of the canonical element to $A_{p, \Phi} \otimes \mathcal{F}^{k}\left(\mathbb{A}\left(z^{0}\right)\right)$. By Lemma 2.6, we have

$$
[E] \in A_{p, \Phi} \otimes \operatorname{Sing} \mathcal{F}^{k}\left(\mathbb{A}\left(z^{0}\right)\right) .
$$

Theorem 7.9. We have

(i) $S^{(a)}([E],[E])=(-1)^{k}\left[\operatorname{Hess}^{(a)}\right]$,

(ii) $\left(1 \otimes \operatorname{pr} K_{j}^{1}\left(z^{0}\right)\right)[E]=\left(\left[a_{j} / f_{j}(z, \cdot)\right] \otimes 1\right)[E]$ for $j \in J$.

Proof. Part (i) follows from Theorem 2.8. Part (ii) follows from Theorem 7.8. 


\section{Geometric interpretation of the algebra of Hamiltonians}

\subsection{An abstract setting}

Let $k<n$ be positive integers and $J=\{1, \ldots, n\}$ as before.

Let $F$ be a germ of a holomorphic function at a point $p \in \mathbb{C}^{k}$. Assume that $p$ is an isolated critical point of $F$ with Milnor number $\mu_{p}$. Let $A_{p, F}$ be the local algebra of the critical point and $(,)_{p}$ the residue bilinear form on $A_{p, F}$, see (2.3). Denote by [Hess $F$ ] the projection to $A_{p, F}$ of the germ $\operatorname{det}_{1 \leqslant l, m \leqslant k}\left(\partial^{2} F / \partial t_{l} \partial t_{m}\right)$.

Let $h_{1}, \ldots, h_{\mu_{p}}$ be a $\mathbb{C}$-basis of $A_{p, F}$. Let $g_{1}, \ldots, g_{n} \in A_{p, F}$ be a collection of elements such that the unital subalgebra of $A_{p, F}$ generated by $g_{1}, \ldots, g_{n}$ equals $A_{p, F}$.

Let $W$ be a vector space with a symmetric bilinear form $S$. Let $M_{j}: W \rightarrow W, j \in J$, be a collection of commuting symmetric linear operators,

$$
M_{i} M_{j}=M_{j} M_{i}, \quad S\left(M_{j} u, v\right)=S\left(u, M_{j} v\right) \quad \text { for all } i, j \in J \text { and } u, v \in W .
$$

Assume that an element

$$
w=\sum_{l=1}^{\mu_{p}} h_{l} \otimes w_{l} \in A_{p, F} \otimes W
$$

is given such that

$$
\begin{aligned}
& \sum_{l=1}^{\mu_{p}} h_{l} \otimes M_{j} w_{l}=\sum_{l=1}^{m} g_{j} h_{l} \otimes w_{l}, \quad j \in J, \\
& \sum_{l, m=1}^{\mu_{p}} S\left(w_{l}, w_{m}\right) h_{l} h_{m}=(-1)^{k}[\operatorname{Hess} F] .
\end{aligned}
$$

Denote by $Y \subset W$ the vector subspace generated by $w_{1}, \ldots, w_{\mu_{p}}$. By property (8.1), every $M_{j}$, $j \in J$, preserves $Y$. Denote by $A_{Y}$ the unital subalgebra of $\operatorname{End}(Y)$ generated by $\left.M_{j}\right|_{Y}, j \in J$. The subspace $Y$ is an $A_{Y}$-module. Define a linear map

$$
\alpha: A_{p, F} \rightarrow Y, \quad f \mapsto \sum_{l=1}^{\mu_{p}}\left(f, h_{l}\right)_{p} w_{l} .
$$

\section{Theorem 8.1.}

(i) The map $\alpha: A_{p, F} \rightarrow Y$ is an isomorphism of vector spaces. The form $S$ restricted to $Y$ is nondegenerate.

(ii) The map $\left.g_{j} \mapsto M_{j}\right|_{Y}, j \in J$, extends uniquely to an algebra isomorphism $\beta: A_{p, F} \rightarrow A_{Y}$.

(iii) The maps $\alpha, \beta$ give an isomorphism of the regular representation of $A_{p, F}$ and the $A_{Y-}$ module $Y$, that is $M_{j} \alpha(f)=\alpha\left(g_{j} f\right)$ for any $f \in A_{p, F}$ and $j \in J$.

(iv) Define the value $w(p)$ of $w$ at $p$ as the image of $w$ under the natural projection $A_{p, F} \otimes W \rightarrow$ $A_{p, F} / \mathfrak{m}_{p} \otimes W=W$. Then $w(p)=\alpha(\operatorname{Hess} F) / \mu_{p}$ and the value $w(p)$ is nonzero. The vector $w(p)$ is the only (up to proportionality) common eigenvector of the operators $\left.M_{j}\right|_{Y}, j \in J$, and we have $M_{j} w(p)=g_{j}(p) w(p)$.

This theorem is an analog of Theorem 5.5 and Corollary 5.6 in [15]. The proof is analogous to the proofs in [15]. 


\subsection{Proof of Theorem 8.1}

Lemma 8.2. We have $M_{j} \alpha(f)=\alpha\left(g_{j} f\right)$ for any $f \in A_{p, F}, j \in J$.

Proof. We have $M_{j} \alpha(f)=\sum_{l=1}^{\mu_{p}}\left(f, h_{l}\right)_{p} M_{j} w_{l}$. By (8.1), that is equal to $\sum_{l=1}^{m}\left(f, g_{j} h_{l}\right)_{p} w_{l}=$ $\sum_{l=1}^{m}\left(g_{j} f, h_{l}\right)_{p} w_{l}=\alpha\left(g_{j} f\right)$.

Define a bilinear form $(,)_{S}$ on $A_{p, F}$,

$$
(f, g)_{S}=S(\alpha(f), \alpha(g))=\sum_{l, m=1}^{\mu_{p}} S\left(w_{l}, w_{m}\right)\left(f, h_{l}\right)_{p}\left(g, h_{m}\right)_{p}
$$

Lemma 8.3. We have $(f g, h)_{S}=(f, g h)_{S}$ for all $f, g, h \in A_{p, F}$.

Proof. Since $g_{j}, j \in J$, generate $A_{p, F}$ it is enough to show that $\left(f g_{j}, h\right)_{S}=\left(f, g_{j} h\right)_{S}$ for all $f, h \in A_{p, F}, j \in J$. We have $\left(f g_{j}, h\right)_{S}=S\left(\alpha\left(f g_{j}\right), \alpha(h)\right)=S\left(M_{j} \alpha(f), \alpha(g)\right)=S\left(\alpha(f), M_{j} \alpha(g)\right)$ $=S\left(\alpha(f), \alpha\left(g_{j} h\right)\right)=\left(f, g_{j} h\right)_{S}$.

Lemma 8.4. There exists a unique element $s \in A_{p, F}$ such that $(f, g)_{S}=(s f, g)_{p}$ for all $f, g \in$ $A_{p, F}$.

Proof. Consider the linear function $A_{p, F} \rightarrow \mathbb{C}, f \mapsto(1, f)_{S}$. Since the bilinear form $(,,)_{p}$ is nondegenerate there exists a unique $s \in A_{p, F}$ such that $(1, f)_{S}=(s, f)_{p}$ for any $f \in A_{p, F}$. Hence for any $f, g \in A_{p, F}$ we have $(f, g)_{S}=(1, f g)_{S}=(s, f g)_{p}=(s f, g)_{p}$.

Lemma 8.5. For any $f \in A_{p, F}$, the trace of the linear operator $L_{f}: A_{p, F} \rightarrow A_{p, F}, h \mapsto f h$, is given by the formula $\operatorname{tr} L_{f}=(f$, [Hess $\left.F]\right)_{p}$.

Proof. We have $\operatorname{tr} L_{1}=\mu_{p}=(1$, [Hess $\left.F]\right)_{p}$ and $\operatorname{tr} L_{f}=0=(f$, [Hess $\left.F]\right)_{p}$ for any $f \in \mathfrak{m}_{p}$. This proves the lemma.

Let $h_{1}^{*}, \ldots, h_{\mu_{p}}^{*}$ be a $\mathbb{C}$-basis of $A_{p, F}$ dual to $h_{1}, \ldots, h_{\mu_{p}}$ with respect to the form $(,)_{p}$. Then $\sum_{l=1}^{\mu_{p}}\left([\operatorname{Hess} F], h_{l}^{*}\right)_{p} h_{l}=[$ Hess $F]$. Indeed, for any $f \in A_{p, F}$ we have $\sum_{l=1}^{\mu_{p}}\left(f, h_{l}^{*}\right)_{p} h_{l}=f$.

Lemma 8.6. We have $\sum_{l=1}^{\mu_{p}} h_{l} h_{l}^{*}=[\operatorname{Hess} F]$.

Proof. For any $f \in A_{p, F}$, we have $\operatorname{tr} L_{f}=\sum_{l=1}^{\mu_{p}}\left(h_{l}^{*}, f h_{l}\right)_{p}=\sum_{l=1}^{\mu_{p}}\left(h_{l}^{*} h_{l}, f\right)_{p}$ and $\operatorname{tr} L_{f}=(f,[\text { Hess } F])_{p}$. This proves the lemma.

Lemma 8.7. Let $s \in A_{p, F}$ be the element defined in Lemma 8.4. Then $s$ has the following two properties:

(i) the element $s$ is invertible and projects to $(-1)^{k}$ in $\mathbb{C}=A_{p, F} / \mathfrak{m}_{p}$,

(ii) the form $(,)_{S}$ is nondegenerate.

Proof. To prove the lemma it is enough to show that $(-1)^{k}[\operatorname{Hess} F]=s[\operatorname{Hess} F]$. Indeed, on one hand we have

$$
(f, g)_{S}=\sum_{l, m=1}^{\mu_{p}} S\left(w_{l}, w_{m}\right)\left(f, h_{l}\right)_{p}\left(g, h_{m}\right)_{p} .
$$


On the other hand we have

$$
(f, g)_{S}=(s f, g)_{p}=\sum_{l=1}^{\mu_{p}}\left(s f, h_{l}\right)_{p}\left(g, h_{l}^{*}\right)_{p} .
$$

Hence, $\sum_{l, m=1}^{\mu_{p}} S\left(w_{l}, w_{m}\right) h_{l} \otimes h_{m}=\sum_{l=1}^{\mu_{p}} s h_{l} \otimes h_{l}^{*}$ in $A_{p, F} \otimes A_{p, F}$. Therefore, $\sum_{l, m=1}^{\mu_{p}} S\left(w_{l}, w_{m}\right) h_{l} h_{m}=$ $\sum_{l=1}^{\mu_{p}} s h_{l} h_{l}^{*}$. By Assumption (8.2) and Lemma 8.6 we obtain $(-1)^{k}[\operatorname{Hess} F]=s[\operatorname{Hess} F]$.

Let us prove Theorem 8.1. Assume that $\sum_{l=1}^{\mu_{p}} \lambda_{l} w_{l}=0$ with $\lambda_{l} \in \mathbb{C}$. Denote $h=\sum_{l=1}^{\mu_{p}} \lambda_{l} h_{l}^{*}$. Then $\alpha(h)=0$ and $(f, h)_{S}=S(\alpha(f), \alpha(h))=0$ for all $f \in A_{p, F}$. Hence $h=0$ since $(,)_{S}$ is nondegenerate. Therefore, $\lambda_{l}=0$ for all $l$ and the vectors $v_{1}, \ldots, v_{\mu_{\mathcal{T}}}$ are linearly independent. This proves part $(i)$ of Theorem 8.1. Parts $(i i)$ and $(i i i)$ follow from Lemma 8.2.

\subsection{Remark on maximal commutative subalgebras}

Let $A$ be a commutative algebra with unity element 1 . Let $B$ be the subalgebra of $\operatorname{End}(A)$ generated by all multiplication operators $L_{f}: A \rightarrow A, h \mapsto f h$, where $f \in A$.

Lemma 8.8. The subalgebra $B$ is a maximal commutative subalgebra of $\operatorname{End}(A)$.

Proof. Let $T \in \operatorname{End}(A)$ be such that $\left[T, L_{f}\right]=0$ for all $f \in A$. Then $T=L_{T(1)}$.

Corollary 8.9. Under assumptions of Section 8.1, the algebra $A_{Y}$ is a maximal commutative subalgebra of $\operatorname{End}(Y)$.

\subsection{Interpretation of the algebra of Hamiltonians of good fibers}

Under notations of Section 3 fix a point $z \in \mathbb{C}^{n}-\Delta$. Recall that in formula (5.1) we have defined the quantum integrable model assigned to $(\mathbb{A}(z), a)$ to be the collection

$$
\left(\operatorname{Sing} W ;\left.S^{(a)}\right|_{\operatorname{Sing} W} ;\left.K_{1}(z)^{*}\right|_{\operatorname{Sing} W}, \ldots,\left.K_{n}(z)^{*}\right|_{\operatorname{Sing} W}: \operatorname{Sing} W \rightarrow \operatorname{Sing} W\right) .
$$

Let $p \in U(\mathbb{A}(z))$ be an isolated critical point of the master function $\Phi(z, \cdot): U(\mathbb{A}(z)) \rightarrow \mathbb{C}$. Let $A_{p, \Phi}$ be the local algebra of the critical point and $(,)_{p}$ the residue bilinear form on $A_{p, \Phi}$. Let $[\boldsymbol{E}] \in A_{p, \Phi} \otimes \operatorname{Sing} W$ be the element corresponding to the canonical element, see Section 5.5.

Define a linear map

$$
\alpha_{p}: A_{p, \Phi} \rightarrow \operatorname{Sing} W, \quad g \mapsto(g,[\boldsymbol{E}])_{p} .
$$

Denote $Y_{p}$ the image of $\alpha_{p}$.

\section{Theorem 8.10.}

(i) We have ker $\alpha_{p}=0$.

(ii) The operators $K_{j}(z)^{*}$ preserve $Y_{p}$. Moreover, for any $j \in J, g \in A_{p, \Phi}$, we have

$$
\alpha_{p}\left(g a_{j} /\left[f_{j}(z, \cdot)\right]\right)=K_{j}(z)^{*} \alpha_{p}(g) .
$$


(iii) Define the value $[\boldsymbol{E}](p)$ of $[\boldsymbol{E}]$ at $p$ as the image of $[\boldsymbol{E}]$ under the natural projection $A_{p, \Phi} \otimes$ Sing $W \rightarrow A_{p, \Phi} / \mathfrak{m}_{p} \otimes \operatorname{Sing} W=\operatorname{Sing} W$. Then the value $[\boldsymbol{E}](p)$ is nonzero. The vector $[\boldsymbol{E}](p)$ is the only (up to proportionality) common eigenvector of the operators $\left.K_{j}(z)^{*}\right|_{Y_{p}}$ : $Y_{p} \rightarrow Y_{p}, j \in J$, and we have

$$
K_{j}(z)^{*}[\boldsymbol{E}](p)=\frac{a_{j}}{f_{j}(z, p)}[\boldsymbol{E}](p) .
$$

Proof. By Theorem 5.9 and Remark 5.10 the objects $\operatorname{Sing} W,\left.S^{(a)}\right|_{\operatorname{Sing} W},\left.K_{j}(z)^{*}\right|_{\operatorname{Sing} W},[\boldsymbol{v}]$, $\left[a_{j} / f_{j}(z, \cdot)\right], j \in J$, satisfy the assumptions of Theorem 8.1. Now Theorem 8.10 follows from Theorem 8.1.

Theorem 8.11. The linear map $\alpha_{p}$ identifies the contravariant form on $Y_{p}$ and the residue form $(,)_{p}$ on $A_{p, \Phi}$ multiplied by $(-1)^{k}$,

$$
S^{(a)}\left(\alpha_{p}(f), \alpha_{p}(g)\right)=(-1)^{k}(f, g)_{p}
$$

for any $f, g \in A_{p, \Phi}$.

Proof. If the Milnor number of $p$ is one, then the theorem follows from Lemma 8.7. If the Milnor number is greater than one, the theorem follows by continuity from the case of the Milnor number equal to one, since all objects involved depend continuously on the weights $a$ and parameters $z$.

Note that the theorem says that the element $s$ of Lemma 8.7 in our situation equals $(-1)^{k}$.

Remark 8.12. In formula (8.3), each of $\alpha_{p}(f), \alpha_{p}(g)$ is given by the Grothendieck residue, so each of $\alpha_{p}(f), \alpha_{p}(g)$ is a $k$-dimensional integral. The quantity $(f, g)_{p}$ is also a $k$-dimensional integral. Thus formula (8.3) is an equality relating a bilinear expression in $k$-dimensional integrals to an individual $k$-dimensional integral.

Denote by $A_{Y_{p}}$ the unital subalgebra of $\operatorname{End}\left(Y_{p}\right)$ generated by $\left.K_{j}(z)^{*}\right|_{Y_{p}}, j \in J$.

\section{Corollary 8.13.}

(i) The map $\left.\left[a_{j} / f_{j}(z, \cdot)\right] \mapsto K_{j}(z)^{*}\right|_{Y_{p}}, j \in J$, extends uniquely to an algebra isomorphism $\beta_{p}: A_{p, \Phi} \rightarrow A_{Y_{p}}$.

(ii) The maps $\alpha_{p}, \beta_{p}$ give an isomorphism of the regular representation of $A_{p, \Phi}$ and the $A_{Y_{p}}{ }^{-}$ module $Y_{p}$, that is $\beta_{p}(h) \alpha_{p}(g)=\alpha_{p}(h g)$ for any $h, g \in A_{p, \Phi}$.

(iii) The algebra $A_{Y_{p}}$ is a maximal commutative subalgebra of $\operatorname{End}\left(Y_{p}\right)$.

(iv) All elements of the algebra $A_{Y_{p}}$ are symmetric operators with respect to the contravariant form $S^{(a)}$.

Theorem 8.14. Let $p_{1}, \ldots, p_{d}$ be a list of all distinct isolated critical points of $\Phi(z, \cdot)$. Let $Y_{p_{s}}=\alpha_{p_{s}}\left(A_{p_{s}}, \Phi\right) \subset \operatorname{Sing} W, s=1, \ldots, d$, be the corresponding subspaces. Then the sum of these subspaces is direct. The subspaces are orthogonal.

Proof. It follows from Theorem 8.10 that for any $s=1, \ldots, d$ and $j \in J$ the operator $K_{j}(z)^{*}-$ $a_{j} / f_{j}\left(z, p_{s}\right)$ restricted to $Y_{p_{s}}$ is nilpotent. We also know that the numbers $a_{j} / f_{j}\left(z, p_{s}\right)$ separate the points $p_{1}, \ldots, p_{d}$. These observations imply Theorem 8.14.

Corollary 8.15. The sum of Milnor numbers of the critical points $p_{1}, \ldots, p_{d}$ is not greater than the rank of the contravariant form $\left.S^{(a)}\right|_{\operatorname{Sing} W}$. 
Denote $Y=\oplus_{s=1}^{d} Y_{p_{s}}$. Denote by $A_{Y}$ the unital subalgebra of $\operatorname{End}(Y)$ generated by $\left.K_{j}(z)^{*}\right|_{Y}$, $j \in J$. Consider the isomorphisms

$$
\alpha=\oplus_{s=1}^{d} \alpha_{p_{s}}: \oplus_{s=1}^{d} A_{p_{s}, \Phi} \rightarrow \oplus_{s=1}^{d} Y_{p_{s}}, \quad \beta=\oplus_{s=1}^{d} \beta_{s}: \oplus_{s=1}^{d} A_{p_{s}, \Phi} \rightarrow \oplus_{s=1}^{d} A_{Y_{p_{s}}} .
$$

Corollary 8.16. We have

(i) $A_{Y}=\oplus_{s=1}^{d} A_{p_{s}, Y_{p_{s}}}$;

(ii) $A_{Y}$ is a maximal commutative subalgebra of $\operatorname{End}(Y)$.

(iii) The isomorphisms $\alpha, \beta$ identify the regular representation of the algebra $\oplus_{s=1}^{d} A_{p_{s}, \Phi}$ and the $A_{Y}$-module $Y$. The isomorphism $\alpha$ identifies the contravariant form on $Y$ and the residue form $()=,\oplus_{s=1}^{d}(,)_{p_{s}}$ on $\oplus_{s=1}^{d} A_{p_{s}, \Phi}$ multiplied by $(-1)^{k}$.

(iv) In particular, if the dimension of Sing $W$ equals the sum of Milnor numbers $\sum_{s=1}^{d} \mu_{s}$, then the module Sing $W$ over the unital subalgebra of $\operatorname{End}(\operatorname{Sing} W)$ generated by geometric Hamiltonians $\left.K_{j}(z)^{*}\right|_{\operatorname{Sing} W}: \operatorname{Sing} W \rightarrow \operatorname{Sing} W, j \in J$, is isomorphic to the regular representation of the algebra $\oplus_{s=1}^{d} A_{p_{s}, \Phi}$.

(v) If for $z \in \mathbb{C}^{n}-\Delta$ the arrangement $(\mathbb{A}(z), a)$ is unbalanced, then the module Sing $W$ over the unital subalgebra of End(Sing $W$ ) generated by geometric Hamiltonians $\left.K_{j}(z)^{*}\right|_{\operatorname{Sing} W}$, $j \in J$, is isomorphic to the regular representation of the algebra $\oplus_{s=1}^{d} A_{p_{s}, \Phi}$.

Corollary 8.17. If for $z \in \mathbb{C}^{n}-\Delta$ the arrangement $(\mathbb{A}(z), a)$ is unbalanced, then the contravariant form is nondegenerate on $\operatorname{Sing} \mathcal{F}^{k}(\mathbb{A}(z))$.

Proof. Indeed in this case the sum of Milnor numbers of critical points of the master function equals $|\chi(U(\mathbb{A}(z)))|$ and equals $\operatorname{dim} \operatorname{Sing} \mathcal{F}^{k}(\mathbb{A}(z))$.

\subsection{Interpretation of the algebra of Hamiltonians of bad fibers if Assumption 7.4 is satisfied}

Let Assumption 7.4 be satisfied. Fix $z^{0} \in \Delta$. We have defined the quantum integrable model assigned to $\left(\mathbb{A}\left(z^{0}\right), a\right)$ to be the collection

$$
\begin{aligned}
& \left(\operatorname{Sing} \mathcal{F}^{k}\left(\mathbb{A}\left(z^{0}\right)\right) ;\left.S^{(a)}\right|_{\operatorname{Sing} \mathcal{F}^{k}\left(\mathbb{A}\left(z^{0}\right)\right)}\right. \\
& \left.\tilde{K}_{1}\left(z^{0}\right), \ldots, \tilde{K}_{n}\left(z^{0}\right): \operatorname{Sing} \mathcal{F}^{k}\left(\mathbb{A}\left(z^{0}\right)\right) \rightarrow \operatorname{Sing} \mathcal{F}^{k}\left(\mathbb{A}\left(z^{0}\right)\right)\right),
\end{aligned}
$$

where $\tilde{K}_{j}\left(z^{0}\right)=\left.\operatorname{pr} K_{j}^{1}\left(z^{0}\right)\right|_{\operatorname{Sing} \mathcal{F}^{k}\left(\mathbb{A}\left(z^{0}\right)\right)}$, see Theorem 7.5.

Let $p \in U\left(\mathbb{A}\left(z^{0}\right)\right)$ be an isolated critical point of the master function $\Phi\left(z^{0}, \cdot\right): U\left(\mathbb{A}\left(z^{0}\right)\right) \rightarrow \mathbb{C}$. Let $A_{p, \Phi}$ be the local algebra of the critical point and $(,)_{p}$ the residue bilinear form on $A_{p, \Phi}$. Let $[E] \in A_{p, \Phi} \otimes \operatorname{Sing} \mathcal{F}^{k}\left(\mathbb{A}\left(z^{0}\right)\right)$ be the canonical element corresponding to the arrangements $\mathbb{A}\left(z^{0}\right)$, see Section 7.8 .

Define a linear map

$$
\alpha_{p}: A_{p, \Phi} \rightarrow \operatorname{Sing} \mathcal{F}^{k}\left(\mathbb{A}\left(z^{0}\right)\right), \quad g \mapsto(g,[E])_{p} .
$$

Denote $Y_{p}$ the image of $\alpha_{p}$.

Theorem 8.18. Let Assumption 7.4 be satisfied. Then

(i) We have ker $\alpha_{p}=0$. The isomorphism $\alpha_{p}$ identifies the contravariant form on $Y_{p}$ and the residue form $(,)_{p}$ on $A_{p, \Phi}$ multiplied by $(-1)^{k}$. 
(ii) For any $j \in J$, the operator $\tilde{K}_{j}\left(z^{0}\right)$ preserves $Y_{p}$. Moreover, for any $j \in J$ and $g \in A_{p, \Phi}$, we have $\alpha_{p}\left(g a_{j} /\left[f_{j}\left(z^{0}, \cdot\right)\right]\right)=\tilde{K}_{j}\left(z^{0}\right) \alpha_{p}(g)$.

(iii) Define the value $[E](p)$ of $[E]$ at $p$ as the image of $[E]$ under the natural projection $A_{p, \Phi} \otimes$ $\operatorname{Sing} \mathcal{F}^{k}\left(\mathbb{A}\left(z^{0}\right)\right) \rightarrow A_{p, \Phi} / \mathfrak{m}_{p} \otimes \operatorname{Sing} \mathcal{F}^{k}\left(\mathbb{A}\left(z^{0}\right)\right)=\operatorname{Sing} \mathcal{F}^{k}\left(\mathbb{A}\left(z^{0}\right)\right)$. Then the value $[E](p)$ is nonzero. The vector $[E](p)$ is the only (up to proportionality) common eigenvector of the operators $\left.\tilde{K}_{j}\left(z^{0}\right)\right|_{Y_{p}}: Y_{p} \rightarrow Y_{p}, j \in J$, and we have

$$
\tilde{K}_{j}\left(z^{0}\right)[E](p)=\frac{a_{j}}{f_{j}\left(z^{0}, p\right)}[E](p) .
$$

Denote by $A_{Y_{p}}$ the unital subalgebra of $\operatorname{End}\left(Y_{p}\right)$ generated by $\left.\tilde{K}_{j}\left(z^{0}\right)\right|_{Y_{p}}, j \in J$.

Corollary 8.19. Let Assumption 7.4 be satisfied. Then

(i) The map $\left.\left[a_{j} / f_{j}\left(z^{0}, \cdot\right)\right] \mapsto \tilde{K}_{j}\left(z^{0}\right)\right|_{Y_{p}}, j \in J$, extends uniquely to an algebra isomorphism $\beta_{p}: A_{p, \Phi} \rightarrow A_{Y_{p}}$.

(ii) The maps $\alpha_{p}, \beta_{p}$ give an isomorphism of the regular representation of $A_{p, \Phi}$ and the $A_{Y_{p}}$ module $Y_{p}$, that is $\beta_{p}(h) \alpha_{p}(g)=\alpha_{p}(h g)$ for any $h, g \in A_{p, \Phi}$.

(iii) The algebra $A_{Y_{p}}$ is a maximal commutative subalgebra of $\operatorname{End}\left(Y_{p}\right)$.

Recall that under Assumption 7.4 all critical points of $\Phi\left(z^{0}, \cdot\right)$ are isolated, the sum of their Milnor numbers equals $\operatorname{dim} \operatorname{Sing} \mathcal{F}^{k}\left(\mathbb{A}\left(z^{0}\right)\right)$ and the form $\left.S^{(a)}\right|_{\operatorname{Sing} \mathcal{F}^{k}\left(\mathbb{A}\left(z^{0}\right)\right)}$ is nondegenerate.

Theorem 8.20. Let Assumption 7.4 be satisfied. Let $p_{1}, \ldots, p_{d}$ be a list of all distinct critical points of $\Phi\left(z^{0}, \cdot\right)$. Let $Y_{p_{s}}=\alpha_{p_{s}}\left(A_{p_{s}, \Phi}\right) \subset \operatorname{Sing} \mathcal{F}^{k}\left(\mathbb{A}\left(z^{0}\right)\right), s=1, \ldots, d$, be the corresponding subspaces. Then the sum of these subspaces is direct, orthogonal and equals $\operatorname{Sing} \mathcal{F}^{k}\left(\mathbb{A}\left(z^{0}\right)\right)$.

Denote by $A_{\mathbb{A}\left(z^{0}\right), a}$ the unital subalgebra of $\operatorname{End}\left(\operatorname{Sing} \mathcal{F}^{k}\left(\mathbb{A}\left(z^{0}\right)\right)\right)$ generated by $\tilde{K}_{j}\left(z^{0}\right), j \in J$. The algebra $A_{\mathbb{A}\left(z^{0}\right), a}$ is called the algebra of geometric Hamiltonian of the arrangement $\left(\mathbb{A}\left(z^{0}\right), a\right)$, see Section 7.3.

Consider the isomorphisms

$$
\begin{aligned}
& \alpha=\oplus_{s=1}^{d} \alpha_{p_{s}}: \oplus_{s=1}^{d} A_{p_{s}, \Phi} \rightarrow \operatorname{Sing} \mathcal{F}^{k}\left(\mathbb{A}\left(z^{0}\right)\right)=\oplus_{s=1}^{d} Y_{p_{s}}, \\
& \beta=\oplus_{s=1}^{d} \beta_{s}: \oplus_{s=1}^{d} A_{p_{s}, \Phi} \rightarrow \oplus_{s=1}^{d} A_{Y_{p_{s}}} .
\end{aligned}
$$

Corollary 8.21. Let Assumption 7.4 be satisfied. Then

(i) $A_{\mathbb{A}\left(z^{0}\right), a}=\oplus_{s=1}^{d} A_{Y_{p_{s}}}$.

(ii) $A_{\mathbb{A}\left(z^{0}\right), a}$ is a maximal commutative subalgebra of $\operatorname{End}\left(\operatorname{Sing} \mathcal{F}^{k}\left(\mathbb{A}\left(z^{0}\right)\right)\right)$.

(iii) The isomorphisms $\alpha, \beta$ identify the regular representation of the algebra $\oplus_{s=1}^{d} A_{p_{s}, \Phi}$ and the $A_{\mathbb{A}\left(z^{0}\right), a}$-module $\operatorname{Sing} \mathcal{F}^{k}\left(\mathbb{A}\left(z^{0}\right)\right)$. The isomorphism $\alpha$ identifies the contravariant form on Sing $\mathcal{F}^{k}\left(\mathbb{A}\left(z^{0}\right)\right)$ and the residue form $()=,\oplus_{s=1}^{d}(,)_{p_{s}}$ on $\oplus_{s=1}^{d} A_{p_{s}, \Phi}$ multiplied by $(-1)^{k}$.

\section{More on Hamiltonians of bad fibers}

\subsection{An abstract setting}

Let $k<n$ be positive integers and $J=\{1, \ldots, n\}$ as before.

Let $B \subset \mathbb{C}^{k}$ be a ball with center at a point $p$. Let $F_{u}$ be a holomorphic function on $B$ depending holomorphically on a complex parameter $u$ at $u=0$. Assume that $F_{0}=F_{u=0}$ has a single 
critical point at $p$ with Milnor number $\mu$. Let $\mathbb{C}(t)_{B}$ be the algebra of holomorphic functions on $B, I_{u} \subset \mathbb{C}(t)_{B}$ the ideal generated by $\partial F_{u} / \partial t_{i}, i=1, \ldots, k$, and $A_{u}=\mathbb{C}(t)_{B} / I_{u}$. Assume that $\operatorname{dim}_{\mathbb{C}} A_{u}$ does not depend on $u$ for $u$ in a neighborhood of 0 . Let []$_{u}: \mathbb{C}(t)_{B} \rightarrow A_{u}$ be the canonical projection, $(,)_{u}$ the residue bilinear form on $A_{u}$ and Hess $F_{u}=\operatorname{det}_{1 \leqslant l, m \leqslant k}\left(\partial^{2} F_{u} / \partial t_{l} \partial t_{m}\right)$.

Let $h_{1}, \ldots, h_{\mu} \in \mathbb{C}(t)_{B}$ be a collection of elements such that for any $u$ the elements $\left[h_{1}\right]_{u}, \ldots$, $\left[h_{\mu}\right]_{u}$ form a $\mathbb{C}$-basis of $A_{u}$.

Let $g_{1, u}, \ldots, g_{n, u} \in \mathbb{C}(t)_{B}$ be elements depending on $u$ holomorphically at $u=0$ and such that for any $u$ (close to 0 ) the unital subalgebra of $A_{u}$ generated by $\left[g_{1, u}\right]_{u}, \ldots,\left[g_{n, u}\right]_{u}$ equals $A_{u}$.

Let $W$ be a vector space with a symmetric bilinear form $S$. For $u \neq 0$, let $M_{j, u}: W \rightarrow W$, $j \in J$, be a collection of commuting symmetric linear operators,

$$
M_{i, u} M_{j, u}=M_{j, u} M_{i, u}, \quad S\left(M_{j, u} x, y\right)=S\left(x, M_{j, u} y\right) \quad \text { for all } i, j \in J \text { and } x, y \in W .
$$

We assume that every $M_{j, u}$ depends on $u$ meromorphically (for $u$ close to 0 ) and has at most simple pole at $u=0$,

$$
M_{j, u}=\frac{M_{j}^{(-1)}}{u}+M_{j}^{(0)}+M_{j ; 1}^{(1)} u+\cdots, \quad M_{j}^{(i)} \in \operatorname{End}(W) .
$$

Let $w_{1, u}, \ldots, w_{\mu, u} \in W$ be a collection of vectors depending on $u$ holomorphically at $u=0$.

Consider the element

$$
[w]_{u}=\sum_{l=1}^{\mu}\left[h_{l}\right]_{u} \otimes w_{l, u} \in A_{u} \otimes W .
$$

Assume that for every nonzero $u$ (close to 0 ) we have

$$
\sum_{l=1}^{\mu}\left[h_{l}\right]_{u} \otimes M_{j, u} w_{l, u}=\sum_{l=1}^{\mu}\left[g_{j, u}\right]_{u}\left[h_{l}\right]_{u} \otimes w_{l, u}, \quad j \in J,
$$

and for every $u$ (close to 0 ) we have

$$
\sum_{l, m=1}^{\mu_{p}} S\left(w_{l, u}, w_{m, u}\right)\left[h_{l}\right]_{u}\left[h_{m}\right]_{u}=(-1)^{k}\left[\operatorname{Hess} F_{u}\right]_{u}
$$

For any $u$, denote by $Y_{u} \subset W$ the vector subspace generated by $w_{1, u}, \ldots, w_{\mu, u}$. By property (9.2), for any nonzero $u$ (close to 0 ) every $M_{j, u}, j \in J$, preserves $Y_{u}$. For any nonzero $u$ (close to 0 ) denote by $A_{Y_{u}}$ the unital subalgebra of $\operatorname{End}\left(Y_{u}\right)$ generated by $\left.M_{j, u}\right|_{Y_{u}}, j \in J$. The subspace $Y_{u}$ is an $A_{Y_{u}}$-module.

For any $u(u=0$ included) define a linear map

$$
\alpha_{u}: A_{u} \rightarrow Y_{u}, \quad[f]_{u} \mapsto \sum_{l=1}^{\mu}\left([f]_{u},\left[h_{l}\right]_{u}\right)_{u} w_{l, u}
$$

Theorem 9.1. For any $u$ (in particular, for $u=0$ ) the map $\alpha_{u}: A_{u} \rightarrow Y_{u}$ is an isomorphism of vector spaces. The form $S$ restricted to $Y_{u}$ is nondegenerate.

Proof. Define a bilinear form $(,)_{S, u}$ on $A_{u}$,

$$
\left([f]_{u},[g]_{u}\right)_{S, u}=S\left(\alpha_{u}\left([f]_{u}\right), \alpha_{u}\left([g]_{u}\right)\right)=\sum_{l, m=1}^{\mu} S\left(w_{l, u}, w_{m, l}\right)\left([f]_{u},\left[h_{l}\right]_{u}\right)_{u}\left([g]_{u},\left[h_{m}\right]_{u}\right)_{u} .
$$


Lemma 9.2. For any $u$, we have $\left([f]_{u}[g]_{u},[h]_{u}\right)_{S, u}=\left([f]_{u},[g]_{u}[h]_{u}\right)_{S, u}$ for all $[f]_{u},[g]_{u},[h]_{u} \in A_{u}$.

Proof. For $u \neq 0$, the statement follows from Lemma 8.3. For $u=0$, the statement follows by continuity.

The next two lemmas are similar to the corresponding analogs in Section 8.2.

Lemma 9.3. There exists a unique element $[s]_{0} \in A_{0}$ such that $\left([f]_{0},[g]_{0}\right)_{S, 0}=\left([s]_{0}[f]_{0},[g]_{0}\right)_{0}$ for all $[f]_{0},[g]_{0} \in A_{0}$.

Lemma 9.4. Let $[s]_{0} \in A_{0}$ be the element defined in Lemma 9.3. Then $[s]_{0}$ has the following two properties:

(i) the element $[s]_{0}$ is invertible and projects to $(-1)^{k}$ in $\mathbb{C}=A_{0} / \mathfrak{m}_{0}$, where $\mathfrak{m}_{0} \subset A_{0}$ is the maximal ideal,

(ii) the form $(,)_{S, 0}$ is nondegenerate.

Lemma 9.4 implies Theorem 9.1, cf. the end of Section 8.2.

Define the value $[w]_{0}(p)$ of $[w]_{0}$ at $p$ as the image of $[w]_{0}$ under the natural projection $A_{0} \otimes W \rightarrow A_{0} / \mathfrak{m}_{0} \otimes W=W$.

Corollary 9.5. The value $[w]_{0}(p)$ is nonzero.

Corollary 9.6. The space $Y_{0}$ is of dimension $\mu$ and $w_{1,0}, \ldots, w_{l, 0}$ is its basis.

For any $[g]_{0} \in A_{0}$, denote by $L_{[g]_{0}} \in \operatorname{End}\left(A_{0}\right)$ the linear operator on $A_{0}$ of multiplication by $[g]_{0}$. For any $j \in J$, define a linear map $\bar{L}_{j, 0}: Y_{0} \rightarrow Y_{0}$ by the formula $\bar{L}_{j, 0}=\alpha_{0} L_{\left[g_{j, 0}\right]_{0}}\left(\alpha_{0}\right)^{-1}$. Denote by $A_{Y_{0}}$ the unital subalgebra of $\operatorname{End}\left(Y_{0}\right)$ generated by $\bar{L}_{j, 0}, j \in J$. Clearly, $A_{Y_{0}}$ is commutative. The subspace $Y_{0}$ is an $A_{Y_{0}}$-module.

\section{Theorem 9.7.}

(i) The map $\left[g_{j, 0}\right]_{0} \mapsto \bar{L}_{j, 0}, j \in J$, extends uniquely to an algebra isomorphism $\beta_{0}: A_{0} \rightarrow A_{Y_{0}}$.

(ii) the algebra $A_{Y_{u}}$ tends to the algebra $A_{Y_{0}}$ as $u \rightarrow 0$. More precisely, for any $j \in J$ and $l=1, \ldots, \mu$, we have $M_{j, u} w_{l, u} \rightarrow \bar{L}_{j, 0} w_{l, 0}$ as $u \rightarrow 0$.

Proof. Part $(i)$ is clear. Part $(i i)$ follows from (9.2).

Let $\tilde{Y} \subset W$ be a vector subspace such that

(a) $Y_{0} \subset \tilde{Y}$;

(b) the bilinear form $S$ restricted on $\tilde{Y}$ is nondegenerate;

(c) for any $j \in J$, the subspace $\tilde{Y}$ lies in the kernel of $M_{j}^{(-1)}$.

For example, we can choose $\tilde{Y}=Y_{0}$. Let $\operatorname{pr}_{\tilde{Y}}: W \rightarrow \tilde{Y}$ be the orthogonal projection.

\section{Theorem 9.8.}

(i) For $j \in J$, let $M_{j}^{(0)}$ be the constant coefficient of the Laurent expansion of $M_{j, u}$, see (9.1). Then

$$
\bar{L}_{j, 0}=\left.\operatorname{pr}_{\tilde{Y}} M_{j}^{(0)}\right|_{Y_{0}} .
$$

In particular, that means that the operators $\left.\operatorname{pr}_{\tilde{Y}} M_{j}^{(0)}\right|_{Y_{0}}$ do not depend on the choice of $\tilde{Y}$. 
(ii) Let $\left(\xi_{j}\right)_{j \in J} \subset \mathbb{C}$ be numbers such that $\sum_{j \in J} \xi_{j} M_{j}^{(-1)}=0$, then

$$
\sum_{j \in J} \xi_{j} \bar{L}_{j, 0}=\left.\sum_{j \in J} \xi_{j} M_{j}^{(0)}\right|_{Y_{0}}
$$

Proof. For $j \in J$, let $w_{l, u}=w_{l, 0}+w_{l}^{(1)} u+\cdots$ be the Taylor expansion of $w_{l, u}$. By part (ii) of Theorem 9.7 we have

$$
w_{j, 0} \in \operatorname{ker} M_{j}^{(-1)} \quad \text { and } \quad \bar{L}_{j, 0} w_{l, 0}=M_{j}^{(0)} w_{l, 0}+M_{j}^{(-1)} w_{l, 0}^{(1)} .
$$

The operator $M_{j}^{(-1)}$ is symmetric since $M_{j, u}$ is symmetric. The image of $M_{j}^{(-1)}$ is orthogonal to the kernel of $M_{j}^{(-1)}$. Hence, formula (9.3) follows from formula (9.4). Part (ii) of the theorem also follows from formula (9.4).

Corollary 9.9. For any $i, j \in J$, the operators $\left.\operatorname{pr}_{\tilde{Y}} M_{i}^{(0)}\right|_{Y_{0}},\left.\operatorname{pr}_{\tilde{Y}} M_{j}^{(0)}\right|_{Y_{0}}$ are symmetric and commute.

Corollary 9.10. The unital subalgebra of $\operatorname{End}\left(Y_{0}\right)$ generated by the operators $\left.\operatorname{pr}_{\tilde{Y}} M_{j}^{(0)}\right|_{Y_{0}}$, $j \in J$, is a maximal commutative subalgebra.

Proof. The proof follows from remarks in Section 8.3.

Corollary 9.11. The vector $[w]_{0}(p)$ is the only (up to proportionality) common eigenvector of the operators $\left.\operatorname{pr}_{\tilde{Y}} M_{j}^{(0)}\right|_{Y_{0}}, j \in J$, and we have $\operatorname{pr}_{\tilde{Y}} M_{j}^{(0)}[w]_{0}(p)=g_{j, 0}(p)[w]_{0}(p)$.

Assume that the parameter $u$ is changed holomorphically, $u=c_{1} v+c_{2} v^{2}+\cdots$ where $c_{i} \in \mathbb{C}$, $c_{1} \neq 0$, and $v$ is a new parameter. Let

$$
M_{j, u(v)}=\frac{\tilde{M}_{j}^{(-1)}}{v}+\tilde{M}_{j}^{(0)}+\tilde{M}_{j ; 1}^{(1)} v+\cdots, \quad \tilde{M}_{j}^{(i)} \in \operatorname{End}(W),
$$

be the new Laurent expansion.

Lemma 9.12. For any $j \in J$, we have $\left.\operatorname{pr}_{\tilde{Y}} M_{i}^{(0)}\right|_{Y_{0}}=\left.\operatorname{pr}_{\tilde{Y}} \tilde{M}_{i}^{(0)}\right|_{Y_{0}}$ and the algebra $A_{Y_{0}} \subset \operatorname{End}\left(Y_{0}\right)$ does not change under the reparametrization of $u$.

Proof. One proof of the lemma follows from Theorem 9.7. Another proof follows from the fact that $\tilde{M}_{i}^{(-1)}=M_{i}^{(-1)} / c_{1}, \tilde{M}_{i}^{(0)}=M_{i}^{(0)}-c_{2} M_{i}^{(-1)} / c_{1}^{2}$.

\subsection{Hamiltonians of bad fibers}

Let us return to the situation of Sections 3 and 7 and recall the previous constructions.

Let $z^{0} \in \Delta$. Let $V \rightarrow V^{*}$ be the map associated with the contravariant form. Let $W$, Sing $W$, $W\left(z^{0}\right)$, Sing $W\left(z^{0}\right)$ be the images of $V$, Sing $V, \mathcal{F}^{k}\left(\mathbb{A}\left(z^{0}\right)\right)$, Sing $\mathcal{F}^{k}\left(\mathbb{A}\left(z^{0}\right)\right)$, respectively. The contravariant form on $V$ induces a nondegenerate symmetric bilinear form on $W$ also denoted by $S^{(a)}$.

For $z \in \mathbb{C}^{n}-\Delta$, we have linear operators $K_{j}(z): V \rightarrow V, j \in J$, where $K_{j}(z)=\sum_{C \in \mathfrak{C}} \frac{\lambda_{j}^{C}}{f_{C}(z)} L_{C}$, see (5.3). For $\mathfrak{C}_{0}=\left\{C \in \mathfrak{C} \mid z^{0} \in H_{C}\right\}$, we define

$$
K_{j}^{0}(z)=\sum_{C \in \mathfrak{C}_{0}} \frac{\lambda_{j}^{C}}{f_{C}(z)} L_{C}, \quad K_{j}^{1}(z)=K_{j}(z)-K_{j}^{0}(z) .
$$


The dual operators $K_{j}(z)^{*}: V^{*} \rightarrow V^{*}$ preserve the subspaces Sing $W \subset W \subset V^{*}$, commute on the subspace Sing $W$ and are symmetric on $W$ with respect to the contravariant form. The operators $L_{C}^{*}: V^{*} \rightarrow V^{*}, C \in \mathfrak{C}$, preserve the subspaces Sing $W \subset W \subset V^{*}$. The space $W\left(z^{0}\right)$ lies in the kernel of $\left.L_{C}^{*}\right|_{W}: W \rightarrow W$ for any $C \in \mathfrak{C}_{0}$.

Let $T_{z^{0}}=\left\{\xi=\sum_{j \in J} \xi_{j} \frac{\partial}{\partial z_{j}} \mid \xi_{j} \in \mathbb{C}, \xi\left(f_{C}\right)=0\right.$ for all $\left.C \in \mathfrak{C}_{0}\right\}$.

Let $p \in U\left(\mathbb{A}\left(z^{0}\right)\right)$ be an isolated critical point of the master function $\Phi\left(z^{0}, \cdot\right): U\left(\mathbb{A}\left(z^{0}\right)\right) \rightarrow \mathbb{C}$. Let $A_{p, \Phi}$ be the local algebra of the critical point and $(,)_{p}$ the residue bilinear form on $A_{p, \Phi}$. Let [ ] : $\mathbb{C}(t)_{U\left(\mathbb{A}\left(z^{0}\right)\right)} \rightarrow A_{p, \Phi}$ be the canonical projection and $[\boldsymbol{E}] \in A_{p, \Phi} \otimes \operatorname{Sing} W\left(z^{0}\right)$ the element corresponding to the canonical element.

Define a linear map

$$
\alpha_{p}: A_{p, \Phi} \rightarrow \operatorname{Sing} W\left(z^{0}\right), \quad g \mapsto(g,[\boldsymbol{E}])_{p} .
$$

Denote $Y_{p}$ the image of $\alpha_{p}$.

\section{Theorem 9.13.}

(i) We have ker $\alpha_{p}=0$. The isomorphism $\alpha_{p}$ identifies the contravariant form on $Y_{p}$ and the residue form $(,)_{p}$ on $A_{p, \Phi}$ multiplied by $(-1)^{k}$. In particular, the contravariant form on $Y_{p}$ is nondegenerate.

(ii) Let $\tilde{Y} \subset$ Sing $W$ be a vector subspace such that

(a) $Y_{p} \subset \tilde{Y}$;

(b) the contravariant form restricted on $\tilde{Y}$ is nondegenerate;

(c) for any $j \in J$, the subspace $\tilde{Y}$ lies in the kernel of $L_{C}^{*}, C \in \mathfrak{C}_{0}$.

Let $\operatorname{pr}_{\tilde{Y}}: \operatorname{Sing} W \rightarrow \tilde{Y}$ be the orthogonal projection. Then for any $j \in J$ the operator $\left.\operatorname{pr}_{\tilde{Y}} K_{j}^{1}\left(z^{0}\right)^{*}\right|_{Y_{p}}$ maps $Y_{p}$ to $Y_{p}$ and does not depend on the choice of $\tilde{Y}$. The operators $\left.\operatorname{pr}_{\tilde{Y}} K_{j}^{1}\left(z^{0}\right)^{*}\right|_{Y_{p}}: Y_{p} \rightarrow Y_{p}, j \in J$, commute and are symmetric with respect to the contravariant form on $Y_{p}$.

(iii) The unital subalgebra $A_{Y_{p}} \subset \operatorname{End}\left(Y_{p}\right)$ generated by $\left.\operatorname{pr}_{\tilde{Y}} K_{j}^{1}\left(z^{0}\right)^{*}\right|_{Y_{p}}, j \in J$, is a maximal commutative subalgebra.

(iv) The naive geometric Hamiltonians $K_{\xi}\left(z^{0}\right)^{*}, \xi \in T_{z^{0}}$, preserve the subspace $Y_{p}$ and the operators $\left.K_{\xi}\left(z^{0}\right)^{*}\right|_{Y_{p}}$ are elements of the subalgebra $A_{Y_{p}}$.

(v) The value $[\boldsymbol{E}](p)$ of $[\boldsymbol{E}]$ at $p$ is nonzero. The vector $[\boldsymbol{E}](p)$ is the only (up to proportionality) common eigenvector of the operators $\left.\operatorname{pr}_{\tilde{Y}} K_{j}^{1}\left(z^{0}\right)^{*}\right|_{Y_{p}}, j \in J$, and we have

$$
\operatorname{pr}_{\tilde{Y}} K_{j}^{1}\left(z^{0}\right)^{*}[\boldsymbol{E}](p)=\frac{a_{j}}{f_{j}\left(z^{0}, p\right)}[\boldsymbol{E}](p)
$$

(vi) For any $j \in J, g \in A_{p, \Phi}$, we have $\alpha_{p}\left(g a_{j} /\left[f_{j}\left(z^{0}, \cdot\right)\right]\right)=\operatorname{pr}_{\tilde{Y}} K_{j}^{1}\left(z^{0}\right)^{*} \alpha_{p}(g)$. The map $\left.\left[a_{j} / f_{j}\left(z^{0},\right)\right] \mapsto \operatorname{pr}_{\tilde{Y}} K_{j}^{1}\left(z^{0}\right)^{*}\right|_{Y_{p}}, j \in J$, extends uniquely to an algebra isomorphism $\beta_{p}$ : $A_{p, \Phi} \rightarrow A_{Y_{p}}$.

(vii) The isomorphisms $\alpha_{p}$, $\beta_{p}$ identify the regular representation of the algebra $A_{p, \Phi}$ and the $A_{Y_{p}}$-module $Y_{p}$.

All statements of the theorem (but the second statement of part $(i)$ ) follow from the corresponding statements of Section 9.1. The second statement of part $(i)$ has the same proof as Theorem 8.11. 
Theorem 9.14. Let $p_{1}, \ldots, p_{d}$ be a list of all distinct isolated critical points of $\Phi\left(z^{0}, \cdot\right)$. Let $Y_{p_{s}}=\alpha_{p_{s}}\left(A_{p_{s}, \Phi}\right) \subset \operatorname{Sing} W\left(z^{0}\right), s=1, \ldots, d$, be the corresponding subspaces. Then the sum of these subspaces is direct and orthogonal with respect to the contravariant form.

Corollary 9.15. The sum of Milnor numbers of the critical points $p_{1}, \ldots, p_{d}$ is not greater than the rank of the contravariant form $\left.S^{(a)}\right|_{\operatorname{Sing} \mathcal{F}^{k}\left(\mathbb{A}\left(z^{0}\right)\right)}$.

Denote $Y=\oplus_{s=1}^{d} Y_{p_{s}}$. Let $\tilde{Y} \subset \operatorname{Sing} W$ be a vector subspace such that

(a) $Y \subset \tilde{Y}$;

(b) the contravariant form restricted on $\tilde{Y}$ is nondegenerate;

(c) for any $j \in J$, the subspace $\tilde{Y}$ lies in the kernel of $L_{C}^{*}, C \in \mathfrak{C}_{0}$.

For example, we can choose $\tilde{Y}=Y$. Let $\operatorname{pr}_{\tilde{Y}}: \operatorname{Sing} W \rightarrow \tilde{Y}$ be the orthogonal projection.

\section{Theorem 9.16.}

(i) For any $j \in J$ the operator $\operatorname{pr}_{\tilde{Y}} K_{j}^{1}\left(z^{0}\right)^{*}$ maps $Y$ to $Y$ and does not depend on the choice of $\tilde{Y}$. The operators $\left.\operatorname{pr}_{\tilde{Y}} K_{j}^{1}\left(z^{0}\right)^{*}\right|_{Y}: Y \rightarrow Y, j \in J$, commute, preserve each of the subspaces $Y_{p_{s}}$ and are symmetric with respect to the contravariant form on $Y$.

Denote by $A_{Y}$ be the unital subalgebra of $\operatorname{End}(Y)$ generated by $\left.\operatorname{pr}_{\tilde{Y}} K_{j}^{1}\left(z^{0}\right)^{*}\right|_{Y}, j \in J$.

(ii) The naive geometric Hamiltonians $K_{\xi}\left(z^{0}\right)^{*}, \xi \in T_{z^{0}}$, preserve the subspace $Y$ and the operators $\left.K_{\xi}\left(z^{0}\right)^{*}\right|_{Y}$ are elements of the subalgebra $A_{Y}$.

(iii) Consider the isomorphisms

$$
\begin{aligned}
& \alpha=\oplus_{s=1}^{d} \alpha_{p_{s}}: \oplus_{s=1}^{d} A_{p_{s}, \Phi} \rightarrow \oplus_{s=1}^{d} Y_{p_{s}}, \\
& \beta=\oplus_{s=1}^{d} \beta_{s}: \oplus_{s=1}^{d} A_{p_{s}, \Phi} \rightarrow \oplus_{s=1}^{d} A_{Y_{p_{s}}} .
\end{aligned}
$$

Then

(a) $A_{Y}=\oplus_{s=1}^{d} A_{Y_{p_{s}}}$;

(b) $A_{Y}$ is a maximal commutative subalgebra of $\operatorname{End}(Y)$;

(c) the isomorphisms $\alpha, \beta$ identify the regular representation of the algebra $\oplus_{s=1}^{d} A_{p_{s}, \Phi}$ and the $A_{Y}$-module $Y$; the isomorphism $\alpha$ identifies the contravariant form on $Y$ and the residue form $()=,\oplus_{s=1}^{d}(,)_{p_{s}}$ on $\oplus_{s=1}^{d} A_{p_{s}, \Phi}$ multiplied by $(-1)^{k}$;

(d) in particular, if the rank of the contravariant form $\left.S^{(a)}\right|_{\operatorname{Sing} \mathcal{F}^{k}\left(z^{0}\right)}$ equals the sum of Milnor numbers of the points $p_{1}, \ldots, p_{d}$, then $Y=\operatorname{Sing} W\left(z^{0}\right)$ and the module Sing $W\left(z^{0}\right)$ over the unital subalgebra of $\operatorname{End}\left(\operatorname{Sing} W\left(z^{0}\right)\right)$ generated by geometric Hamiltonians $\left.\operatorname{pr}_{\tilde{Y}} K_{j}^{1}\left(z^{0}\right)^{*}\right|_{\operatorname{Sing} W\left(z^{0}\right)}, j \in J$, is isomorphic to the regular representation of the algebra $\oplus_{s=1}^{d} A_{p_{s}, \Phi}$;

The theorem follows from the corresponding statements of Section 9.1.

Theorem 9.17. Assume that the arrangement $\left(\mathbb{A}\left(z^{0}\right), a\right)$ is unbalanced.

(i) Then the contravariant form is nondegenerate on Sing $W\left(z^{0}\right)$.

(ii) Let $\operatorname{pr}_{\operatorname{Sing} W\left(z^{0}\right)}:$ Sing $W \rightarrow \operatorname{Sing} W\left(z^{0}\right)$ be the orthogonal projection, $A_{\operatorname{Sing} W\left(z^{0}\right)}$ be the unital subalgebra of End $\left(\operatorname{Sing} W\left(z^{0}\right)\right)$ generated by the operators $\left.\operatorname{pr}_{\operatorname{Sing} W\left(z^{0}\right)} K_{j}^{1}\left(z^{0}\right)^{*}\right|_{\operatorname{Sing} W\left(z^{0}\right)}$, $j \in J$. Then $A_{\operatorname{Sing} W\left(z^{0}\right)}$ is commutative and its elements are symmetric with respect to the contravariant form on Sing $W\left(z^{0}\right)$. 
(iii) Let $p_{1}, \ldots, p_{d}$ be a list of all distinct isolated critical points of $\Phi\left(z^{0}, \cdot\right)$. Then the $A_{\operatorname{Sing} W\left(z^{0}\right)^{-}}$ module Sing $W\left(z^{0}\right)$ is isomorphic to the regular representation of the algebra $\oplus_{s=1}^{d} A_{p_{s}, \Phi}$.

Proof. If $\left(\mathbb{A}\left(z^{0}\right), a\right)$ is unbalanced, then the sum of Milnor numbers of the master function equals $\left|\chi\left(U\left(\mathbb{A}\left(z^{0}\right)\right)\right)\right|$ and equals $\operatorname{dim} \operatorname{Sing} \mathcal{F}^{k}\left(\mathbb{A}\left(z^{0}\right)\right)$. This implies part $(i)$ of the theorem. Parts $(i i)$ and $($ iii) follow from Theorem 9.16.

\subsection{Remark on critical points of real arrangements}

Assume that $\left(g_{j}\right)_{j \in J}$ are real, see Remark 3.2. Assume that $z^{0} \in \mathbb{R}^{n} \subset \mathbb{C}^{n}$. Assume that the contravariant form is positive definite on Sing $W\left(z^{0}\right)$. Let $\operatorname{pr}_{\operatorname{Sing} W\left(z^{0}\right)}: \operatorname{Sing} W \rightarrow \operatorname{Sing} W\left(z^{0}\right)$ be the orthogonal projection. Assume that the operators

$$
\left.\operatorname{pr}_{\operatorname{Sing} W\left(z^{0}\right)} K_{j}^{1}\left(z^{0}\right)^{*}\right|_{\operatorname{Sing} W\left(z^{0}\right)}: \operatorname{Sing} W\left(z^{0}\right) \rightarrow \operatorname{Sing} W\left(z^{0}\right), \quad j \in J,
$$

commute and are symmetric with respect to the contravariant form.

Theorem 9.18. Under these assumptions, any critical point $p$ of the master function $\Phi\left(z^{0}, \cdot\right)$ : $U\left(\mathbb{A}\left(z^{0}\right)\right) \rightarrow U\left(\mathbb{A}\left(z^{0}\right)\right)$, is nondegenerate and the nonzero value $[\boldsymbol{E}](p)$ at $p$ of the canonical element lies in the real part Sing $W\left(z^{0}\right)_{\mathbb{R}}$ of Sing $W\left(z^{0}\right)$ (up to multiplication by a nonzero complex number).

Proof. On one hand, under assumptions of the theorem all the linear operators preserve Sing $W\left(z^{0}\right)_{\mathbb{R}}$ and can be diagonalized simultaneously. That means that any element of the algebra of geometric Hamiltonians $A_{\operatorname{Sing} W\left(z^{0}\right)}$ (generated by operators $\left.\operatorname{pr}_{\operatorname{Sing} W\left(z^{0}\right)} K_{j}^{1}\left(z^{0}\right)^{*}\right|_{\operatorname{Sing} W\left(z^{0}\right)}$, $j \in J)$ is diagonalizable. On the other hand, if the Milnor number of $p$ is greater than one, then the local algebra $A_{p, \Phi}$ has nilpotent elements and, by Theorem 9.13 , the algebra $A_{\operatorname{Sing} W\left(z^{0}\right)}$ has nondiagonalizable elements.

The second part of the theorem is clear.

Theorem 9.18 is in the spirit of the main theorem of [12] and Conjecture 5.1 in [11]. The main theorem of [12] says that certain Schubert cycles intersect transversally and all intersection point are real. These two statements correspond to the two statements of Theorem 9.18.

\section{Arrangements with symmetries}

\subsection{A family of prediscriminantal arrangements}

In this section we consider a special family of parallelly translated hyperplanes, see Section 3. The members of that family will be called prediscriminantal arrangements.

Data 10.1. Let $\mathfrak{h}^{*}$ be a complex vector space of dimension $r$ with a collection of vectors $\alpha_{1}, \ldots, \alpha_{r}, \Lambda_{1}, \ldots, \Lambda_{N} \in \mathfrak{h}^{*}$ and a symmetric bilinear form $($,$) . We assume that \left(\alpha_{i}, \alpha_{i}\right) \neq 0$ for every $i=1, \ldots, r$.

Let $\boldsymbol{k}=\left(k_{1}, \ldots, k_{r}\right)$ be a collection of nonnegative integers. We denote $k=\sum_{i} k_{i}$ and assume that $k>0$. We assume that for every $b=1, \ldots, N$ there exists $i$ such that $\left(\alpha_{i}, \Lambda_{b}\right) \neq 0$ and $k_{i}>0$.

Consider the expressions

$$
\begin{aligned}
& f_{(i), l, l^{\prime}}=t_{l}^{(i)}-t_{l^{\prime}}^{(i)}+z_{(i), l, l^{\prime}} \quad \text { such that } i=1, \ldots, r \text { and } 1 \leqslant l<l^{\prime} \leqslant k_{i} ; \\
& f_{\left(i, i^{\prime}\right), l, l^{\prime}}=t_{l}^{(i)}-t_{l^{\prime}}^{\left(i^{\prime}\right)}+z_{\left(i, i^{\prime}\right), l, l^{\prime}} \\
& \quad \text { such that } 1 \leqslant i<i^{\prime} \leqslant r, 1 \leqslant l \leqslant k_{i}, 1 \leqslant l^{\prime} \leqslant k_{i^{\prime}} \text { and }\left(\alpha_{i}, \alpha_{i^{\prime}}\right) \neq 0 ; \\
& f_{(i, b), l}=-t_{l}^{(i)}+z_{(i, b), l} \quad \text { such that } 1 \leqslant i \leqslant r, 1 \leqslant l \leqslant k_{i}, 1 \leqslant b \leqslant N \text { and }\left(\alpha_{i}, \Lambda_{b}\right) \neq 0 .
\end{aligned}
$$


Let $J$ denote the set of all low indices of the letters $f$ in these expressions. So $J$ is the union of three nonintersecting subsets $J_{1}, J_{2}, J_{3}$ where $J_{1}$ consists of triples $\left\{(i), l, l^{\prime}\right\}$ from the first line of $(10.1), J_{2}$ consists of four-tuples $\left\{\left(i, i^{\prime}\right), l, l^{\prime}\right\}$ from the second line, $J_{3}$ consists of triples $\{(i, b), l\}$ from the third line. Let $n$ be the number of elements in $J$.

Consider $\mathbb{C}^{k}$ with coordinates

$$
t=\left(t_{1}^{(1)}, \ldots, t_{k_{1}}^{(1)}, \ldots, t_{1}^{(r)}, \ldots, t_{k_{r}}^{(r)}\right) .
$$

Consider $\mathbb{C}^{n}$ with coordinates $z=\left(z_{j}\right)_{j \in J}$ and $\mathbb{C}^{n} \times \mathbb{C}^{k}$ with coordinates $z, t$. For any $j \in J$ the expression $f_{j}$ can be considered as a linear function on $\mathbb{C}^{n} \times \mathbb{C}^{k}$. We have $f_{j}=z_{j}+g_{j}$ where $g_{j}=t_{l}^{(i)}-t_{l^{\prime}}^{(i)}$ if $j=\left\{(i), l, l^{\prime}\right\}$, where $g_{j}=t_{l}^{(i)}-t_{l^{\prime}}^{\left(i^{\prime}\right)}$ if $j=\left\{\left(i, i^{\prime}\right), l, l^{\prime}\right\}$ and $g_{j}=-t_{l}^{(i)}$ if $j=\{(i, b), l\}$. The functions $g_{j}, j \in J$, can be considered as linear functions on $\mathbb{C}^{k}$.

For $j \in J$, the equation $f_{j}(z, t)=0$ defines a hyperplane $\tilde{H}_{j} \subset \mathbb{C}^{n} \times \mathbb{C}^{k}$ and we get an arrangement $\tilde{\mathbb{A}}=\left\{\tilde{H}_{j} \mid j \in J\right\}$ in $\mathbb{C}^{n} \times \mathbb{C}^{k}$.

We assign (nonzero) weights $a_{j}$ to hyperplanes of $\tilde{C}$ by putting

$$
a_{(i), l, l^{\prime}}=\left(\alpha_{i}, \alpha_{i}\right), \quad a_{\left(i, i^{\prime}\right), l, l^{\prime}}=\left(\alpha_{i}, \alpha_{i^{\prime}}\right), \quad a_{(i, b), l}=-\left(\alpha_{i}, \Lambda_{b}\right) .
$$

The weighted arrangement $\tilde{C}$ is an example of a family of parallelly translated hyperplanes considered in Sections 3-9.

\subsection{Discriminantal arrangements}

Let $X \subset \mathbb{C}^{n}$ be the subset defined by the following equations:

$$
\begin{aligned}
& z_{(i), l, l^{\prime}}=0, \quad i=1, \ldots, r \text { and } 1 \leqslant l<l^{\prime} \leqslant k_{i} ; \\
& z_{\left(i, i^{\prime}\right), l, l^{\prime}}=0, \quad 1 \leqslant i<i^{\prime} \leqslant r, 1 \leqslant l \leqslant k_{i}, 1 \leqslant l^{\prime} \leqslant k_{i^{\prime}} \text { and }\left(\alpha_{i}, \alpha_{i^{\prime}}\right) \neq 0 \\
& z_{(i, b), l}=z_{\left(i^{\prime}, b\right), l^{\prime}}, \quad 1 \leqslant i \leqslant r, 1 \leqslant l \leqslant k_{i}, 1 \leqslant i^{\prime} \leqslant r, 1 \leqslant l^{\prime} \leqslant k_{i^{\prime}}, 1 \leqslant b \leqslant N, \\
& \quad\left(\alpha_{i}, \Lambda_{b}\right) \neq 0,\left(\alpha_{i^{\prime}}, \Lambda_{b}\right) \neq 0 .
\end{aligned}
$$

The subset $X$ is an $N$-dimensional affine space. We will use the following coordinates $x_{1}, \ldots, x_{N}$ on $X$ defined by the equations $x_{b}=z_{(i, b), l}$, where $1 \leqslant b \leqslant N, 1 \leqslant i \leqslant r, 1 \leqslant l \leqslant k_{i}$ and $\left(\alpha_{i}, \Lambda_{b}\right) \neq 0$. We will be interested in the open subset $U(X) \subset X$,

$$
U(X)=\left\{z \in X \mid x_{1}(z), \ldots, x_{N}(z) \text { are all distinct }\right\}
$$

Let us consider the arrangement $\mathbb{A}(z)=\left(H_{j}(z)\right)_{j \in J}$ in the fiber $\mathbb{C}^{k}$ of the projection $\mathbb{C}^{n} \times \mathbb{C}^{k} \rightarrow$ $\mathbb{C}^{n}$ over a point $z \in U(X)$ with coordinates $x_{1}(z), \ldots, x_{N}(z)$. Its hyperplanes are defined by the equations:

$$
\begin{aligned}
& t_{l}^{(i)}-t_{l^{\prime}}^{(i)}=0, \quad i=1, \ldots, r \text { and } 1 \leqslant l<l^{\prime} \leqslant k_{i} ; \\
& t_{l}^{(i)}-t_{l^{\prime}}^{\left(i^{\prime}\right)}=0, \quad 1 \leqslant i<i^{\prime} \leqslant r, 1 \leqslant l \leqslant k_{i}, 1 \leqslant l^{\prime} \leqslant k_{i^{\prime}} \text { and }\left(\alpha_{i}, \alpha_{i^{\prime}}\right) \neq 0 ; \\
& t_{l}^{(i)}-x_{b}(z)=0, \quad 1 \leqslant i \leqslant r, 1 \leqslant l \leqslant k_{i}, 1 \leqslant b \leqslant N \text { and }\left(\alpha_{i}, \Lambda_{b}\right) \neq 0 .
\end{aligned}
$$

The weights of these hyperplanes are defined by formula (10.2). This weighted arrangement is called discriminantal, see [23, 25]. 


\subsection{Symmetries of the family of prediscriminantal arrangements}

The product of symmetric groups $S_{\boldsymbol{k}}=S_{k_{1}} \times \cdots \times S_{k_{r}}$ acts on $\mathbb{C}^{k}$ by permuting coordinates $t_{l}^{(i)}$ with the same upper index. More precisely, a point $p \in \mathbb{C}^{k}$ with coordinates $\left(t_{1}^{(1)}(p), \ldots, t_{k_{1}}^{(1)}(p)\right.$, $\left.\ldots, t_{1}^{(r)}(p), \ldots, t_{k_{r}}^{(r)}(p)\right)$ is mapped by an element $\sigma=\left(\sigma_{1}, \ldots, \sigma_{r}\right)$ to the point with coordinates $\left(t_{\sigma_{a}^{-1}(1)}^{(1)}(p), \ldots, t_{\sigma_{1}^{-1}\left(k_{1}\right)}^{(1)}(p), \ldots, t_{\sigma_{r}^{-1}(1)}^{(r)}(p), \ldots, t_{\sigma_{r}^{-1}\left(k_{r}\right)}^{(r)}(p)\right)$.

The group $S_{\boldsymbol{k}}$ acts also on $\mathbb{C}^{n}$. Namely, an element $\sigma=\left(\sigma_{1}, \ldots, \sigma_{r}\right)$ sends a coordinate $z_{(i), l, l^{\prime}}$ to the coordinate $z_{(i), \sigma_{i}(l), \sigma_{i}\left(l^{\prime}\right)}$ if $\sigma_{i}(l)<\sigma_{i}\left(l^{\prime}\right)$ and to $-z_{(i), \sigma_{i}(l), \sigma_{i}\left(l^{\prime}\right)}$ if $\sigma_{i}(l)>\sigma_{i}\left(l^{\prime}\right)$. An element $\sigma$ sends a coordinate $z_{\left(i, i^{\prime}\right), l, l^{\prime}}$ to $z_{\left(i, i^{\prime}\right), \sigma_{i}(l), \sigma_{i^{\prime}}\left(l^{\prime}\right)}$. An element $\sigma$ sends a coordinate $z_{(i, b), l}$ to the coordinate $z_{(i, b), \sigma_{i}(l)}$.

Clearly every point of $X \subset \mathbb{C}^{n}$ is a fixed point of the $S_{\boldsymbol{k}}$-action.

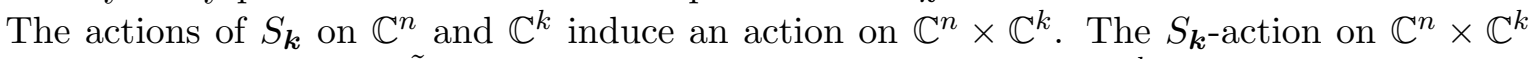
preserves the arrangement $\tilde{\mathbb{A}}$ and sends fibers of the projection $\mathbb{C}^{n} \times \mathbb{C}^{k} \rightarrow \mathbb{C}^{n}$ to fibers.

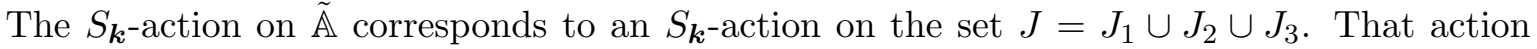
preserves the summands. For $\sigma=\left(\sigma_{1}, \ldots, \sigma_{r}\right) \in S_{\boldsymbol{k}}$ we have

$$
\begin{aligned}
& \left\{(i), l, l^{\prime}\right\} \mapsto\left\{(i), \min \left(\sigma_{i}(l), \sigma_{i}\left(l^{\prime}\right)\right), \max \left(\sigma_{i}(l), \sigma_{i}\left(l^{\prime}\right)\right)\right\}, \\
& \left\{\left(i, i^{\prime}\right), l, l^{\prime}\right\} \mapsto\left\{\left(i, i^{\prime}\right), \sigma_{i}(l), \sigma_{i^{\prime}}\left(l^{\prime}\right)\right\}, \quad\{(i, b), l\} \mapsto\left\{(i, b), \sigma_{i}(l)\right\},
\end{aligned}
$$

where $\left\{(i), l, l^{\prime}\right\} \in J_{1},\left\{\left(i, i^{\prime}\right), l, l^{\prime}\right\} \in J_{2},\{(i, b), l\} \in J_{3}$.

Consider the discriminant $\Delta=\cup_{C \in \mathfrak{C}} H_{C} \subset \mathbb{C}^{n}$, see Section 3.2. Here $\mathfrak{C}$ is the set of all circuits

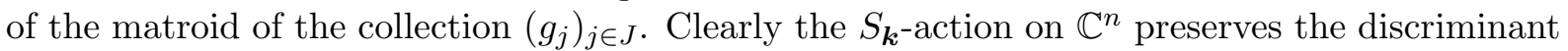
and permutes the hyperplanes $\left\{H_{C} \mid C \in \mathfrak{C}\right\}$.

The action of $S_{\boldsymbol{k}}$ on the hyperplanes of the discriminant corresponds to the following action on $\mathfrak{C}$. If $C=\left\{j_{1}, \ldots, j_{l}\right\} \subset J$ is a circuit and $\sigma \in S_{\boldsymbol{k}}$, then $\sigma(C)$ is the circuit $\left\{\sigma\left(j_{1}\right), \ldots, \sigma\left(j_{l}\right)\right\}$.

\subsection{The $S_{k}$-action on geometric Hamiltonians}

We use notations of Section 3.3 and for $z \in \mathbb{C}^{n}-\Delta$ denote $V=\mathcal{F}^{k}(\mathbb{A}(z))$, Sing $V=\operatorname{Sing} \mathcal{F}^{k}(\mathbb{A}(z))$. The triple $\left(V\right.$, Sing $\left.V, S^{(a)}\right)$ does not depend on $z \in \mathbb{C}^{n}-\Delta$ as explained in Section 3.3.

Fix an order on the set $J$. Recall that the standard basis of $V^{*}=\mathcal{A}^{k}(\mathbb{A}(z))$, associated with an order on $J$, is formed by elements $\left(H_{j_{1}}, \ldots, H_{j_{k}}\right)$ where $\left\{j_{1}<\cdots<j_{k}\right\}$ runs through the set of all independent ordered $k$-element subsets of $J$. The (dual) standard basis of $V$ is formed by the corresponding vectors $F\left(H_{j_{1}}(z), \ldots, H_{j_{k}}(z)\right)$. We have $F\left(H_{j_{1}}(z), \ldots, H_{j_{k}}(z)\right)=$ $(-1)^{|\mu|} F\left(H_{j_{\mu(1)}}(z), \ldots, H_{j_{\mu(k)}}(z)\right)$ for any $\mu \in S_{k}$, see Section 2.11.

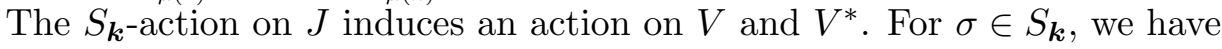

$$
\sigma: F\left(H_{j_{1}}(z), \ldots, H_{j_{k}}(z)\right) \mapsto F\left(H_{\sigma\left(j_{1}\right)}(z), \ldots, H_{\sigma\left(j_{k}\right)}(z)\right)
$$

and $\left(H_{j_{1}}(z), \ldots, H_{j_{k}}(z)\right) \mapsto\left(H_{\sigma\left(j_{1}\right)}(z), \ldots, H_{\sigma\left(j_{k}\right)}(z)\right)$. The $S_{\boldsymbol{k}^{-}}$action on $V$ preserves the subspace Sing $V$ and preserves the contravariant form $S^{(a)}$ on $V$.

Let $z^{0} \in U(X)$. Then $z^{0}$ is $S_{\boldsymbol{k}^{-}}$-invariant and the group $S_{\boldsymbol{k}}$ acts on the fiber $\mathbb{C}^{k}$ over $z^{0}$ and on the weighted arrangement $\left(\mathbb{A}\left(z^{0}\right), a\right)$ in that fiber. The subspaces $\mathcal{F}^{k}\left(\mathbb{A}\left(z^{0}\right)\right)$, Sing $\mathcal{F}^{k}\left(\mathbb{A}\left(z^{0}\right)\right)$ of $V$ are $S_{\boldsymbol{k} \text {-invariant. }}$

Let $V \rightarrow V^{*}$ be the map associated with the contravariant form. Let $W$, Sing $W, W\left(z^{0}\right)$, Sing $W\left(z^{0}\right)$ be the images of $V$, Sing $V, \mathcal{F}^{k}\left(\mathbb{A}\left(z^{0}\right)\right)$, Sing $\mathcal{F}^{k}\left(\mathbb{A}\left(z^{0}\right)\right)$, respectively. All these subspaces are $S_{k}$-invariant.

An $S_{\boldsymbol{k}}$-action on a vector space defines an $S_{\boldsymbol{k}}$-action on linear operators on that space. For $\sigma \in S_{\boldsymbol{k}}$ and a linear operator $L$ we define $\sigma(L)=\sigma L \sigma^{-1}$.

In Section 5.2 we have defined operators $L_{C}: V \rightarrow V, C \in \mathfrak{C}$. Clearly for any $\sigma \in S_{\boldsymbol{k}}$ and any $C \in \mathfrak{C}$ we have $\sigma\left(L_{C}\right)=L_{\sigma(C)}$. 
In Section 5.2 we have considered differential 1 -forms on $\mathbb{C}^{n} \times \mathbb{C}^{k}$ which were denoted by $\omega_{j}$, $j \in J$, and $\omega_{C}, C \in \mathfrak{C}$. The $S_{\boldsymbol{k}}$-action on $\mathbb{C}^{n} \times \mathbb{C}^{k}$ preserves this set of differential 1-forms. Namely for any $j \in J, C \in \mathfrak{C}, \sigma \in S_{\boldsymbol{k}}$, we have $\sigma: \omega_{j} \mapsto \omega_{\sigma(j)}, \omega_{C} \mapsto \omega_{\sigma(C)}$.

Lemma 10.2. The following objects are $S_{\boldsymbol{k}}$-invariant:

$$
\sum_{j \in J} a_{j} \omega_{j}, \quad \sum_{C \in \mathfrak{C}} \omega_{C} \otimes L_{C}, \quad \sum_{\substack{\text { independent } \\\left\{j_{1}<\cdots<j_{k}\right\} \subset J}} \omega_{j_{1}} \wedge \cdots \wedge \omega_{j_{k}} \otimes F\left(H_{j_{1}}, \ldots, H_{j_{k}}\right) .
$$

By the definition of $K_{j}(z): V \rightarrow V, j \in J$, we have

$$
\sum_{C \in \mathfrak{C}} \omega_{C} \otimes L_{C}=\sum_{j \in J} d z_{j} \otimes K_{j}(z)
$$

see formula (5.4). The functions $K_{j}(z)$ are $\operatorname{End}(V)$-valued meromorphic functions on $\mathbb{C}^{n}$.

Since $S_{\boldsymbol{k}}$ acts on $\mathbb{C}^{n}$ and $\operatorname{End}(V)$ it also acts on $\operatorname{End}(V)$-valued functions on $\mathbb{C}^{n}, \sigma: F(q) \mapsto$

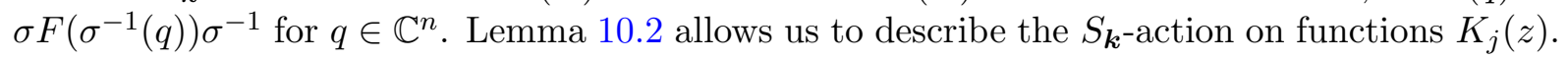

Corollary 10.3. An element $\sigma=\left(\sigma_{1}, \ldots, \sigma_{r}\right) \in S_{\boldsymbol{k}}$ acts on functions $K_{j}, j \in J$, by the formulas:

$$
\begin{aligned}
& K_{(i), l, l^{\prime}}(q) \mapsto K_{(i), \sigma_{i}(l), \sigma_{i}\left(l^{\prime}\right)}\left(\sigma^{-1}(q)\right), \quad \text { if } \sigma_{i}(l)<\sigma_{i}\left(l^{\prime}\right), \\
& K_{(i), l, l^{\prime}}(q) \mapsto-K_{(i), \sigma_{i}\left(l^{\prime}\right), \sigma_{i}(l)}\left(\sigma^{-1}(q)\right), \quad \text { if } \sigma_{i}(l)>\sigma_{i}\left(l^{\prime}\right), \\
& K_{\left(i, i^{\prime}\right), l, l^{\prime}}(q) \mapsto K_{(i), \sigma_{i}(l), \sigma_{i^{\prime}}\left(l^{\prime}\right)}\left(\sigma^{-1}(q)\right), \\
& K_{(i, b), l}(q) \mapsto K_{(i, b), \sigma_{i}(l)}\left(\sigma^{-1}(q)\right) .
\end{aligned}
$$

Let $z^{0} \in U(X)$. Recall that $\mathfrak{C}_{0}=\left\{C \in \mathfrak{C} \mid z^{0} \in H_{C}\right\}$,

$$
K_{j}^{0}(z)=\sum_{C \in \mathfrak{C}_{0}} \frac{\lambda_{j}^{C}}{f_{C}(z)} L_{C}, \quad K_{j}^{1}(z)=K_{j}(z)-K_{j}^{0}(z) .
$$

Corollary 10.4. An element $\sigma \in S_{\boldsymbol{k}}$ acts on operators $K_{j}^{1}\left(z^{0}\right): V \rightarrow V, j \in J$, by the formula

$$
\sigma\left(K_{j}^{1}\left(z^{0}\right)\right)= \pm K_{\sigma(j)}^{1}\left(z^{0}\right)
$$

where the minus sign is chosen only if $j=\left\{(i), l, l^{\prime}\right\}$ and $\sigma_{i}(l)>\sigma\left(l^{\prime}\right)$.

\subsection{Functions $K_{\partial_{x_{b}}}(z)$}

Let $z^{0} \in U(X)$. Recall that $T_{z^{0}}=\left\{\xi=\sum_{j \in J} \xi_{j} \frac{\partial}{\partial z_{j}} \mid \xi_{j} \in \mathbb{C}, \xi\left(f_{C}\right)=0\right.$ for all $\left.C \in \mathfrak{C}_{0}\right\}$, see Section 7.1. Define the following constant vector fields on $\mathbb{C}^{n}$,

$$
\partial_{x_{b}}=\sum_{1 \leqslant i \leqslant r, 1 \leqslant l \leqslant k_{i},\left(\alpha_{i}, \Lambda_{b}\right) \neq 0} \frac{\partial}{\partial z_{(i, b), l}}, \quad b=1, \ldots, N .
$$

Lemma 10.5. The vector fields $\partial_{x_{b}}, b=1, \ldots, N$, are elements of $T_{z^{0}}$. The $\operatorname{End}(V)$-valued functions

$$
K_{\partial_{x_{b}}}(z)=\sum_{1 \leqslant i \leqslant r, 1 \leqslant l \leqslant k_{i},\left(\alpha_{i}, \Lambda_{b}\right) \neq 0} K_{(i, b), l}(z), \quad b=1, \ldots, N,
$$

are $S_{\boldsymbol{k}}$-invariant. 
By Lemma 7.1, the functions $K_{\partial_{y_{b}}}(z)$ are regular at $z^{0}$ and their dual operators preserve the subspace Sing $W\left(z^{0}\right) \subset V^{*}$.

Corollary 10.6. For $z^{0} \in U(X)$, the operators

$$
\left.K_{\partial_{x_{b}}}\left(z^{0}\right)^{*}\right|_{\operatorname{Sing} W\left(z^{0}\right)}: \operatorname{Sing} W\left(z^{0}\right) \rightarrow \operatorname{Sing} W\left(z^{0}\right), \quad b=1, \ldots, N,
$$

are $S_{\boldsymbol{k}}$-invariant.

The operators $\left.K_{\partial_{x_{b}}}\left(z^{0}\right)^{*}\right|_{\operatorname{Sing} W\left(z^{0}\right)}$ are naive geometric Hamiltonians on Sing $W\left(z^{0}\right)$ in the sense of Section 7.1. They commute and they are symmetric operators with respect to the contravariant form.

\subsection{Naive geometric Hamiltonians on Sing $W^{-}\left(z^{0}\right)$}

The space $W$ has the canonical direct sum decomposition into isotypical components corresponding to irreducible representations of $S_{\boldsymbol{k}}$. One of the isotypical components is the component

$$
W^{-}=\left\{x \in W \mid \sigma(x)=(-1)^{|\sigma|} x \text { for any } \sigma \in S_{k}\right\},
$$

corresponding to the alternating representation. If $L: W \rightarrow W$ is an $S_{\boldsymbol{k}}$-invariant linear operator, then $L$ preserves the canonical decomposition and, in particular, it preserves the subspace $W^{-}$.

Let $z^{0} \in U(X)$. Then the subspace Sing $W\left(z^{0}\right) \subset W$ is an $S_{\boldsymbol{k}^{-}}$submodule. We define Sing $W^{-}\left(z^{0}\right)=W^{-} \cap \operatorname{Sing} W\left(z^{0}\right)$. For any $b=1, \ldots, N$, the operator $K_{\partial_{x_{b}}}\left(z^{0}\right)^{*}$ preserves Sing $W^{-}\left(z^{0}\right)$. The operators

$$
\left.K_{\partial_{x_{b}}}\left(z^{0}\right)^{*}\right|_{\operatorname{Sing} W^{-}\left(z^{0}\right)}: \operatorname{Sing} W^{-}\left(z^{0}\right) \rightarrow \operatorname{Sing} W^{-}\left(z^{0}\right)
$$

will be called naive geometric Hamiltonians on $\operatorname{Sing} W^{-}\left(z^{0}\right)$.

\section{7 $S_{k}$-symmetries of the canonical element}

Let $z^{0} \in U(X)$. Let $p \in U\left(\mathbb{A}\left(z^{0}\right)\right)$ be an isolated critical point of the master function $\Phi\left(z^{0}, \cdot\right)$ :

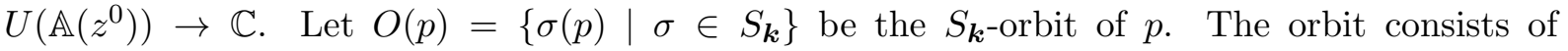
$k_{1} ! \cdots k_{r}$ ! points. Let $A_{\sigma(p), \Phi}$ be the local algebra of the critical point $\sigma(p)$ and $(,)_{\sigma(p)}$ the residue bilinear form on $A_{\sigma(p), \Phi}$. Let []$_{\sigma(p)}: \mathbb{C}(t)_{U\left(\mathbb{A}\left(z^{0}\right)\right)} \rightarrow A_{\sigma(p), \Phi}$ be the canonical projection and $[\boldsymbol{E}]_{\sigma(p)} \in A_{\sigma(p), \Phi} \otimes \operatorname{Sing} W\left(z^{0}\right)$ the projection of the the canonical element.

The group $S_{\boldsymbol{k}}$ acts on functions on $U\left(\mathbb{A}\left(z^{0}\right)\right)$. If $g \in \mathbb{C}(t)_{U\left(\mathbb{A}\left(z^{0}\right)\right)}$ and $\sigma \in S_{\boldsymbol{k}}$, then $\sigma(g)(q)=$ $g\left(\sigma^{-1}(q)\right)$ for $q \in U\left(\mathbb{A}\left(z^{0}\right)\right)$.

Lemma 10.7. An element $\sigma \in S_{\boldsymbol{k}}$ acts on functions $f_{j}, j \in J$, by the formula

$$
\sigma\left(f_{j}\right)= \pm f_{\sigma(j)}
$$

where the minus sign is chosen only if $j=\left\{(i), l, l^{\prime}\right\}$ and $\sigma_{i}(l)>\sigma\left(l^{\prime}\right)$.

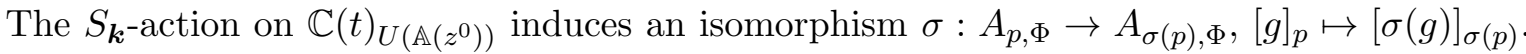
Let us compare the residue bilinear forms on $A_{p, \Phi}$ and $A_{\sigma(p), \Phi}$ and projections of the canonical element to $A_{p, \Phi}$ and $A_{\sigma(p), \Phi}$.

Lemma 10.8. For $f, g \in A_{p, \Phi}$, we have

$$
(\sigma(f), \sigma(g))_{\sigma(p)}=(-1)^{\sigma}(f, g)_{p} \quad \text { and } \quad \sigma\left([\boldsymbol{E}]_{p}\right)=(-1)^{\sigma}[\boldsymbol{E}]_{\sigma(p)},
$$

where $(-1)^{\sigma}=\prod_{i=1}^{r}(-1)^{\sigma_{i}}$. 
For $\sigma \in S_{\boldsymbol{k}}$, let $\alpha_{\sigma(p)}: A_{\sigma(p), \Phi} \rightarrow \operatorname{Sing} W\left(z^{0}\right)$ be the linear monomorphisms constructed in Section 9.2. Let $Y_{\sigma(p)}$ be the image of $\alpha_{\sigma(p)}$. If $\mu$ is the Milnor number of $p$, then $\operatorname{dim} Y_{\sigma(p)}=\mu$.

Lemma 10.9. For $f \in A_{p, \Phi}$, we have $\sigma\left(\alpha_{p}(f)\right)=\alpha_{\sigma(p)}(\sigma(f))$.

Corollary 10.10. We have $\sigma\left(Y_{p}\right)=Y_{\sigma(p)}$.

By Theorem 9.14, the subspaces $Y_{\sigma(p)}$ are all orthogonal and the contravariant form on $Y_{O(p)}=\oplus_{\sigma \in S_{\boldsymbol{k}}} Y_{\sigma(p)}$ is nondegenerate. The group $S_{\boldsymbol{k}}$ acts on $Y_{O(p)}$. Denote

$$
Y_{O(p)}^{-}=\left\{v \in Y_{O(p)} \mid \sigma(v)=(-1)^{\sigma} v \text { for all } \sigma \in S_{\boldsymbol{k}}\right\} .
$$

Corollary 10.11. We have $Y_{O(p)}^{-}=\left\{\sum_{\sigma \in S_{\boldsymbol{k}}}(-1)^{\sigma} \sigma(v) \mid v \in Y_{p}\right\}$ and $\operatorname{dim} Y_{O(p)}^{-}=\mu$. Denote

Let Ant $=\sum_{\sigma \in S_{k}}(-1)^{\sigma} \sigma: \operatorname{Sing} W\left(z^{0}\right) \rightarrow \operatorname{Sing} W^{-}\left(z^{0}\right)$ be the anti-symmetrization operator.

$$
[\boldsymbol{E}]_{p}^{-}=(1 \otimes \mathrm{Ant})[\boldsymbol{E}]_{p}
$$

Define a linear monomorphism

$$
\alpha_{p}^{-}: A_{p, \Phi} \rightarrow \operatorname{Sing} W^{-}\left(z^{0}\right), \quad f \rightarrow\left(f,[\boldsymbol{E}]_{p}^{-}\right)_{p} .
$$

Lemma 10.12. We have

$$
Y_{O(p)}^{-}=\left\{\alpha_{p}^{-}(f) \mid f \in A_{p, \Phi}\right\}
$$

The linear map $\alpha_{p}^{-}$identifies the contravariant form on $Y_{O(p)}^{-}$and the residue bilinear form on $A_{p, \Phi}$ multiplied by $(-1)^{k} k_{1} ! \cdots k_{r} !$. In particular, the contravariant form on $Y_{O(p)}^{-}$is nondegenerate. We also have

$$
\left.S^{(a)}([\boldsymbol{E}])_{p}^{-},[\boldsymbol{E}]_{p}^{-}\right)=(-1)^{k} k_{1} ! \cdots k_{r} !\left[\operatorname{Hess}^{(\mathrm{a})} \Phi\left(z^{0}, \cdot\right)\right]_{p} .
$$

Let $\tilde{Y} \subset \operatorname{Sing} W$ be a vector subspace such that

(a) $Y_{O(p)} \subset \tilde{Y}$;

(b) the contravariant form restricted on $\tilde{Y}$ is nondegenerate;

(c) for any $j \in J$, the subspace $\tilde{Y}$ lies in the kernel of $L_{C}^{*}, C \in \mathfrak{C}_{0}$;

(d) the subspace $\tilde{Y}$ is $S_{\boldsymbol{k}}$-invariant.

For example, we can choose $\tilde{Y}=Y_{O(p)}$. Let $\operatorname{pr}_{\tilde{Y}}:$ Sing $W \rightarrow \tilde{Y}$ be the orthogonal projection with respect to the contravariant form.

For every $j \in J$ and $\sigma \in S_{\boldsymbol{k}}$, the map $\operatorname{pr}_{\tilde{Y}} K_{j}^{1}\left(z^{0}\right)^{*}:$ Sing $W \rightarrow \operatorname{Sing} W$ preserves $Y_{\sigma(p)}$ and $\left.\operatorname{pr}_{\tilde{Y}} K_{j}^{1}\left(z^{0}\right)^{*}\right|_{\tilde{Y}}$ does not depend on the choice of $\tilde{Y}$, see Theorem 9.16. We also have $\sigma: \operatorname{pr}_{\tilde{Y}} K_{j}^{1}\left(z^{0}\right)^{*} \mapsto \pm \operatorname{pr}_{\tilde{Y}} K_{\sigma(j)}^{1}\left(z^{0}\right)^{*}$ where the minus sign is chosen only if $j=\left\{(i), l, l^{\prime}\right\}$ and $\sigma_{i}(l)>\sigma\left(l^{\prime}\right)$.

Important definition. Denote by $P^{S_{k}}$ the algebra of polynomials with complex coefficients in $n$ variables $a_{j} / f_{j}, j \in J$, such that for any $F\left(a_{j} / f_{j}, j \in J\right) \in P^{S_{k}}$ the function $F\left(a_{j} / f_{j}\left(z^{0}, \cdot\right)\right.$, $j \in J) \in U\left(\mathbb{A}\left(z^{0}\right)\right)$ is $S_{\boldsymbol{k}}$-invariant.

Lemma 10.13. The natural homomorphism $P^{S_{k}} \rightarrow A_{p, \Phi}$ is an epimorphism. 
Let $F\left(a_{j} / f_{j}\left(z^{0}, \cdot\right), j \in J\right) \in P^{S_{k}}$. Replace in $F$ each variable $a_{j} / f_{j}$ with the operator $\operatorname{pr}_{\tilde{Y}} K_{j}^{1}\left(z^{0}\right)^{*}$. Denote the resulting operator on $\operatorname{Sing} W$ by $F\left(\operatorname{pr}_{\tilde{Y}} K_{j}^{1}\left(z^{0}\right)^{*}, j \in J\right)$. This operator preserves $Y_{\sigma(p)}$ for any $\sigma \in S_{\boldsymbol{k}}$ and its restriction to $Y_{\sigma(p)}$ does not depend on the choice of $\tilde{Y}$. The

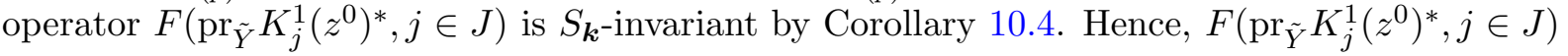
preserves $Y_{O(p)}^{-}$.

\section{Theorem 10.14.}

(i) For $F \in P^{S_{k}}$, the operators

$$
\left.F\left(\operatorname{pr}_{\tilde{Y}} K_{j}^{1}\left(z^{0}\right)^{*}, j \in J\right)\right|_{Y_{O(p)}^{-}}: Y_{O(p)}^{-} \rightarrow Y_{O(p)}^{-}
$$

commute and are symmetric with respect to the contravariant form.

(ii) The map $\left.F \mapsto F\left(\operatorname{pr}_{\tilde{Y}} K_{j}^{1}\left(z^{0}\right)^{*}, j \in J\right)\right|_{Y_{O(p)}^{-}}$induces an algebra monomorphism

$$
\beta_{p}^{-}: A_{p, \Phi} \rightarrow \operatorname{End}\left(Y_{O(p)}^{-}\right)
$$

(iii) The image of this monomorphism, denoted by $A_{Y_{O(p)}^{-}}$, is a maximal commutative subalgebra of $\operatorname{End}\left(Y_{O(p)}^{-}\right)$.

(iv) The naive geometric Hamiltonians $\left.K_{\partial_{x_{b}}}\left(z^{0}\right)^{*}\right|_{Y_{O(p)}^{-}}, b=1, \ldots, N$, are elements of $A_{Y_{O(p)}^{-}}$.

(v) The maps $\alpha_{p}^{-}, \beta_{p}^{-}$define an isomorphism of the regular representation of $A_{p, \Phi}$ and the $A_{Y_{O(p)}^{-}}$-module $Y_{O(p)}^{-}$. The linear map $\alpha_{p}^{-}$identifies the contravariant form on $Y_{O(p)}^{-}$and the residue bilinear form on $A_{p, \Phi}$ multiplied by $(-1)^{k} k_{1} ! \cdots k_{r} !$.

(vi) Define the value of $[\boldsymbol{E}]_{p}^{-}$at $p$ as the image of the natural projection of $[\boldsymbol{E}]_{p}^{-}$to $A_{p, \Phi} / \mathfrak{m}_{p} \otimes$ Sing $W^{-}\left(z^{0}\right)=\operatorname{Sing} W^{-}\left(z^{0}\right)$. Then the value $[\boldsymbol{E}]_{p}^{-}(p)$ is nonzero and lies in $Y_{O(p)}^{-}$. The vector $[\boldsymbol{E}]_{p}^{-}(p)$ is the only (up to proportionality) common eigenvector of the operators $\left.F\left(\operatorname{pr}_{\tilde{Y}} K_{j}^{1}\left(z^{0}\right)^{*}, j \in J\right)\right|_{Y_{O(p)}^{-}}, F \in P^{S_{k}}$, we have

$$
F\left(\operatorname{pr}_{\tilde{Y}} K_{j}^{1}\left(z^{0}\right)^{*}, j \in J\right)[\boldsymbol{E}]_{p}^{-}(p)=F\left(a_{j} / f\left(z^{0}, p\right), j \in J\right)[\boldsymbol{E}]_{p}^{-}(p) .
$$

Theorem 10.15. Let $p_{1}, \ldots, p_{d}$ be a list of isolated critical points of $\Phi\left(z^{0}, \cdot\right)$ such that the orbits $O\left(p_{1}\right), \ldots, O\left(p_{d}\right)$ do not intersect. Let $Y_{p_{s}}^{-}=\alpha_{p_{s}}^{-}\left(A_{p_{s}, \Phi}\right) \subset \operatorname{Sing} W^{-}\left(z^{0}\right), s=1, \ldots, d$, be the corresponding subspaces. Then the sum of these subspaces is direct and orthogonal with respect to the contravariant form.

Corollary 10.16. The sum of Milnor numbers of the critical points $p_{1}, \ldots, p_{d}$ is not greater than the rank of the contravariant form $\left.S^{(a)}\right|_{\operatorname{Sing} W^{-}\left(z^{0}\right)}$.

Let $\tilde{Y} \subset W$ be a vector subspace such that

(a) $\oplus_{s=1}^{d} Y_{O\left(p_{s}\right)} \subset \tilde{Y}$;

(b) the contravariant form restricted on $\tilde{Y}$ is nondegenerate;

(c) for any $j \in J$, the subspace $\tilde{Y}$ lies in the kernel of $L_{C}^{*}, C \in \mathfrak{C}_{0}$;

(d) the subspace $\tilde{Y}$ is $S_{\boldsymbol{k}}$-invariant.

For example, we can choose $\tilde{Y}=\oplus_{s=1}^{d} Y_{O\left(p_{s}\right)}$. Let $\operatorname{pr}_{\tilde{Y}}: \operatorname{Sing} W \rightarrow \tilde{Y}$ be the orthogonal projection.

Denote $Y^{-}=\oplus_{s=1}^{d} Y_{O\left(p_{s}\right)}^{-}$. 
Theorem 10.17. For $F \in P^{S_{k}}$, the operators

$$
\left.F\left(\operatorname{pr}_{\tilde{Y}} K_{j}^{1}\left(z^{0}\right)^{*}, j \in J\right)\right|_{Y^{-}}: Y^{-} \rightarrow Y^{-}
$$

do not depend on the choice of $\tilde{Y}$. They commute and are symmetric with respect to the contravariant form.

Denote by $A_{Y^{-}}$the unital subalgebra of $\operatorname{End}\left(Y^{-}\right)$generated by $\left.F\left(\operatorname{pr}_{\tilde{Y}} K_{j}^{1}\left(z^{0}\right)^{*}, j \in J\right)\right|_{Y^{-}}$, $F \in P^{S_{k}}$. The naive geometric Hamiltonians $\left.K_{\partial_{x_{b}}}\left(z^{0}\right)^{*}\right|_{Y^{-}}, b=1, \ldots, N$, are elements of the algebra $A_{Y^{-}}$.

Consider the isomorphisms

$$
\begin{aligned}
& \alpha^{-}=\oplus_{s=1}^{d} \alpha_{p_{s}}^{-}: \oplus_{s=1}^{d} A_{p_{s}, \Phi} \rightarrow \oplus_{s=1}^{d} Y_{O\left(p_{s}\right)}^{-}, \\
& \beta^{-}=\oplus_{s=1}^{d} \beta_{p_{s}}^{-}: \oplus_{s=1}^{d} A_{p_{s}, \Phi} \rightarrow \oplus_{s=1}^{d} A_{Y_{O\left(p_{s}\right)}^{-}} .
\end{aligned}
$$

Then

(a) $A_{Y^{-}}=\oplus_{s=1}^{d} A_{Y_{O\left(p_{s}\right)}^{-}}$;

(b) $A_{Y^{-}}$is a maximal commutative subalgebra of $\operatorname{End}\left(Y^{-}\right)$;

(c) the isomorphisms $\alpha^{-}, \beta^{-}$identify the regular representation of the algebra $\oplus_{s=1}^{d} A_{p_{s}, \Phi}$ and the $A_{Y^{-}}$-module $Y^{-}$; the linear map $\alpha^{-}$identifies the contravariant form on $Y^{-}$and the residue bilinear form $()=,\oplus_{s=1}^{d}(,)_{p_{s}}$ on $\oplus_{s=1}^{d} A_{p_{s}, \Phi}$ multiplied by $(-1)^{k} k_{1} ! \cdots k_{r} !$;

(d) in particular, if the rank of the contravariant form $\left.S^{(a)}\right|_{\operatorname{Sing} W^{-}\left(z^{0}\right)}$ equals the sum of Milnor numbers of the points $p_{1}, \ldots, p_{d}$, then the module Sing $W^{-}\left(z^{0}\right)=Y^{-}$over the unital subalgebra of $\operatorname{End}\left(\operatorname{Sing} W^{-}\left(z^{0}\right)\right)$ generated by geometric Hamiltonians $F\left(\operatorname{pr}_{\tilde{Y}} K_{j}^{1}\left(z^{0}\right)^{*}, j \in\right.$ $J)\left.\right|_{Y^{-}}, F \in P^{S_{k}}$, is isomorphic to the regular representation of the algebra $\oplus_{s=1}^{d} A_{p_{s}, \Phi}$.

Corollary 10.18. If $z^{0} \in U(X)$ and the arrangement $\left(\mathbb{A}\left(z^{0}\right), a\right)$ is unbalanced, then the operators $\left.F\left(\operatorname{pr}_{\tilde{Y}} K_{j}^{1}\left(z^{0}\right)^{*}, j \in J\right)\right|_{\operatorname{Sing} W^{-}\left(z^{0}\right)}: \operatorname{Sing} W^{-}\left(z^{0}\right) \rightarrow \operatorname{Sing} W^{-}\left(z^{0}\right), F \in P^{S_{k}}$, commute and are symmetric with respect to the contravariant form.

Proof. In this case $Y^{-}=\operatorname{Sing} W^{-}\left(z^{0}\right)$ and the corollary follows from Theorem 10.17.

\section{Applications to the Bethe ansatz of the Gaudin model}

\subsection{Gaudin model}

Let $\mathfrak{g}$ be a simple Lie algebra over $\mathbb{C}$ with Cartan matrix $\left(a_{i, j}\right)_{i, j=1}^{r}$. Let $\mathfrak{h} \subset \mathfrak{g}$ be a Cartan subalgebra. Fix simple roots $\alpha_{1}, \ldots, \alpha_{r}$ in $\mathfrak{h}^{*}$ and a nondegenerate $\mathfrak{g}$-invariant bilinear form (, ) on $\mathfrak{g}$. The form identifies $\mathfrak{g}$ and $\mathfrak{g}^{*}$ and defines a bilinear form on $\mathfrak{g}^{*}$. Let $H_{1}, \ldots, H_{r} \in \mathfrak{h}$ be the corresponding coroots, $\left\langle\lambda, H_{i}\right\rangle=2\left(\lambda, \alpha_{i}\right) /\left(\alpha_{i}, \alpha_{i}\right)$ for $\lambda \in \mathfrak{h}^{*}$. In particular, $\left\langle\alpha_{j}, H_{i}\right\rangle=a_{i, j}$.

Let $E_{1}, \ldots, E_{r} \in \mathfrak{n}_{+}, H_{1}, \ldots, H_{r} \in \mathfrak{h}, F_{1}, \ldots, F_{r} \in \mathfrak{n}_{-}$be the Chevalley generators of $\mathfrak{g}$,

$$
\begin{aligned}
& {\left[E_{i}, F_{j}\right]=\delta_{i, j} H_{i}, \quad i, j=1, \ldots, r,} \\
& {\left[h, h^{\prime}\right]=0, \quad h, h^{\prime} \in \mathfrak{h},} \\
& {\left[h, E_{i}\right]=\left\langle\alpha_{i}, h\right\rangle E_{i}, \quad h \in \mathfrak{h}, i=1, \ldots, r,} \\
& {\left[h, F_{i}\right]=-\left\langle\alpha_{i}, h\right\rangle F_{i}, \quad h \in \mathfrak{h}, i=1, \ldots, r,}
\end{aligned}
$$

and $\left(\operatorname{ad} E_{i}\right)^{1-a_{i, j}} E_{j}=0,\left(\operatorname{ad} F_{i}\right)^{1-a_{i, j}} F_{j}=0$, for all $i \neq j$.

Let $\left(J_{i}\right)_{i \in I}$ be an orthonormal basis of $\mathfrak{g}, \Omega=\sum_{i \in I} J_{i} \otimes J_{i} \in \mathfrak{g} \otimes \mathfrak{g}$ the Casimir element. 
For a $\mathfrak{g}$-module $V$ and $\mu \in \mathfrak{h}^{*}$ denote by $V[\mu]$ the weight subspace of $V$ of weight $\mu$ and by Sing $V[\mu]$ the subspace of singular vectors of weight $\mu$,

$\operatorname{Sing} V[\mu]=\left\{v \in V \mid \mathfrak{n}_{+} v=0, h v=\langle\mu, h\rangle v\right\}$.

Let $N>1$ be an integer and $\boldsymbol{\Lambda}=\left(\Lambda_{1}, \ldots, \Lambda_{N}\right), \Lambda_{b} \in \mathfrak{h}^{*}$, a set of weights. For $\mu \in \mathfrak{h}^{*}$ let $V_{\mu}$ be the irreducible $\mathfrak{g}$-module with highest weight $\mu$. Denote

$$
V_{\Lambda}=V_{\Lambda_{1}} \otimes \cdots \otimes V_{\Lambda_{N}}
$$

For $X \in \operatorname{End}\left(V_{\Lambda_{i}}\right)$, denote by

$$
X^{(i)}=1 \otimes \cdots \otimes 1 \otimes X \otimes 1 \otimes \cdots \otimes 1 \in \operatorname{End}\left(V_{\boldsymbol{\Lambda}}\right)
$$

the operator acting nontrivially on the $i$-th factor only. For $X=\sum_{m} X_{m} \otimes Y_{m} \in \operatorname{End}\left(V_{\Lambda_{i}} \otimes V_{\Lambda_{j}}\right)$, we set $X^{(i, j)}=\sum_{m} X_{m}^{(i)} \otimes Y_{m}^{(j)} \in \operatorname{End}\left(V_{\boldsymbol{\Lambda}}\right)$.

Let $x^{0}=\left(x_{1}^{0}, \ldots, x_{N}^{0}\right)$ be a point of $\mathbb{C}^{N}$ with distinct coordinates. Introduce linear operators $\boldsymbol{K}_{1}\left(x^{0}\right), \ldots, \boldsymbol{K}_{N}\left(x^{0}\right)$ on $V_{\boldsymbol{\Lambda}}$ by the formula

$$
\boldsymbol{K}_{b}\left(x^{0}\right)=\sum_{c \neq b} \frac{\Omega^{(b, c)}}{x_{b}^{0}-x_{c}^{0}}, \quad b=1, \ldots, N .
$$

The operators are called the Gaudin Hamiltonians. The Hamiltonians commute,

$$
\left[\boldsymbol{K}_{b}\left(x^{0}\right), \boldsymbol{K}_{c}\left(x^{0}\right)\right]=0 \quad \text { for all } b, c .
$$

The Hamiltonians commute with the $\mathfrak{g}$-action on $V_{\boldsymbol{\Lambda}}$. Hence they preserve the subspaces Sing $V_{\boldsymbol{\Lambda}}[\mu] \subset V_{\boldsymbol{\Lambda}}$ of singular vectors of a given weight $\mu$.

Let $\tau: \mathfrak{g} \rightarrow \mathfrak{g}$ be the anti-involution sending $E_{i}, H_{i}, F_{i}$, to $F_{i}, H_{i}, E_{i}$, respectively, for all $i$. Let $W$ be a highest weight $\mathfrak{g}$-module with a highest weight vector $w$. The Shapovalov form $S$ on $W$ is the unique symmetric bilinear form such that

$$
S(w, w)=1, \quad S(g u, v)=S(u, \tau(g) v)
$$

for all $u, v \in W$ and $g \in \mathfrak{g}$.

Fix highest weight vectors $v_{1}, \ldots, v_{N}$ of $V_{\Lambda_{1}}, \ldots, V_{\Lambda_{N}}$, respectively. Define a symmetric bilinear form on the tensor product $V_{\boldsymbol{\Lambda}}$ by the formula

$$
S_{\Lambda}=S_{1} \otimes \cdots \otimes S_{N}
$$

where $S_{b}$ is the Shapovalov form on $V_{\Lambda_{b}}$. The form $S_{\boldsymbol{\Lambda}}$ is called the tensor Shapovalov form.

The Gaudin Hamiltonians are symmetric with respect to the tensor Shapovalov form, $S_{\boldsymbol{\Lambda}}\left(\boldsymbol{K}_{b}(y) u, v\right)=S_{\boldsymbol{\Lambda}}\left(u, \boldsymbol{K}_{b}(y) v\right)$ for any $u, v \in V_{\boldsymbol{\Lambda}}$ and $b=1, \ldots, N$, see [19].

For any $\mu \in \mathfrak{h}^{*}$, the Gaudin model on Sing $V_{\boldsymbol{\Lambda}}[\mu] \subset V_{\boldsymbol{\Lambda}}$ is the collection

$$
\left(\operatorname{Sing} V_{\boldsymbol{\Lambda}}[\mu] ;\left.S_{\boldsymbol{\Lambda}}\right|_{\operatorname{Sing} V_{\boldsymbol{\Lambda}}[\mu]} ;\left.\boldsymbol{K}_{b}(y)\right|_{\operatorname{Sing} V_{\boldsymbol{\Lambda}}[\mu]}: \operatorname{Sing} V_{\boldsymbol{\Lambda}}[\mu] \rightarrow \operatorname{Sing} V_{\boldsymbol{\Lambda}}[\mu], b=1, \ldots, N\right),
$$

see $[7,8]$. The main problem for the Gaudin model is to find common eigenvectors and eigenvalues of the Gaudin Hamiltonians. 


\subsection{Master function and weight function, [23]}

The eigenvectors of the Gaudin Hamiltonians are constructed by the Bethe ansatz method. We remind that construction in this section.

Fix a collection of nonnegative integers $\boldsymbol{k}=\left(k_{1}, \ldots, k_{r}\right)$. Denote $k=k_{1}+\cdots+k_{r}$,

$$
\Lambda_{\infty}=\sum_{b=1}^{N} \Lambda_{b}-\sum_{i=1}^{r} k_{i} \alpha_{i}
$$

Consider $\mathbb{C}^{k}$ with coordinates

$$
t=\left(t_{1}^{(1)}, \ldots, t_{k_{1}}^{(1)}, \ldots, t_{1}^{(r)}, \ldots, t_{k_{r}}^{(r)}\right) .
$$

Define the master function

$$
\begin{aligned}
\Phi\left(x^{0}, t, \boldsymbol{\Lambda}, \boldsymbol{k}\right) & =\sum_{i=1}^{r} \sum_{1 \leqslant j<j^{\prime} \leqslant k_{i}}\left(\alpha_{i}, \alpha_{i}\right) \log \left(t_{j}^{(i)}-t_{j^{\prime}}^{(i)}\right) \\
& +\sum_{1 \leqslant i<i^{\prime} \leqslant r} \sum_{j=1}^{k_{i}} \sum_{j^{\prime}=1}^{k_{i^{\prime}}}\left(\alpha_{i}, \alpha_{i^{\prime}}\right) \log \left(t_{j}^{(i)}-t_{j^{\prime}}^{\left(i^{\prime}\right)}\right)-\sum_{i=1}^{r} \sum_{j=1}^{k_{i}} \sum_{b=1}^{N}\left(\Lambda_{b}, \alpha_{i}\right) \log \left(t_{j}^{(i)}-x_{b}^{0}\right) .
\end{aligned}
$$

We consider $\Phi$ as a function of $t$ depending on parameters $x^{0}$.

Denote by $U$ the set of all points $p \in \mathbb{C}^{k}$ such that for any $\log h$ entering (11.1) (with a nonzero coefficient) we have $h(p) \neq 0$. The set $U$ is the complement in $\mathbb{C}^{k}$ to the union of hyperplanes. The coefficients of the logarithms in (11.1) define weights of the hyperplanes. This weighted arrangement is called discriminantal. Discriminantal arrangements were considered in Section 10.2 .

Let us construct the weight function $\omega: \mathbb{C}^{k} \rightarrow V_{\boldsymbol{\Lambda}}\left[\Lambda_{\infty}\right]$ introduced in [23], cf. [20]. Let $b=\left(b_{1}, \ldots, b_{N}\right)$ be a sequence of nonnegative integers with $\sum_{i=1}^{N} b_{i}=k$. The set of all such sequences will be denoted by $B$.

For $b \in B$, let $\left\{c_{1}^{1}, \ldots, c_{b_{1}}^{1}, \ldots, c_{1}^{N}, \ldots, c_{b_{N}}^{N}\right\}$ be a set of letters. Let $\Sigma(b)$ be the set of all bijections $\sigma$ from the set $\left\{c_{1}^{1}, \ldots, c_{b_{1}}^{1}, \ldots, c_{1}^{N}, \ldots, c_{b_{N}}^{N}\right\}$ to the set of variables $\left\{t_{1}^{(1)}, \ldots, t_{k_{1}}^{(1)}, \ldots\right.$, $\left.t_{1}^{(r)}, \ldots, t_{k_{r}}^{(r)}\right\}$. Denote $d\left(t_{j}^{(i)}\right)=i$, and $d_{\sigma, b}=\left(d_{1}^{1}, \ldots, d_{b_{1}}^{1}, \ldots, d_{1}^{N}, \ldots, d_{b_{N}}^{N}\right)$, where $d_{l}^{m}=d\left(\sigma\left(c_{l}^{m}\right)\right)$.

To each $b \in B$ and $\sigma \in \Sigma(b)$ we assign the vector

$$
F_{d_{\sigma, b}} v=F_{d_{1}^{1}} \cdots F_{d_{b_{1}}^{1}} v_{1} \otimes \cdots \otimes F_{d_{1}^{N}} \cdots F_{d_{b_{N}}^{N}} v_{N} \in V_{\Lambda}\left[\Lambda_{\infty}\right] .
$$

and the rational function

$$
\omega_{\sigma, b}=\omega_{\sigma, b}^{1}\left(x_{1}^{0}\right) \cdots \omega_{\sigma, b}^{N}\left(x_{N}^{0}\right),
$$

with

$$
\omega_{\sigma, b}^{e}\left(x_{e}^{0}\right)=\frac{1}{\left(\sigma\left(c_{1}^{e}\right)-\sigma\left(c_{2}^{e}\right)\right) \cdots\left(\sigma\left(c_{b_{e}-1}^{e}\right)-\sigma\left(c_{b_{e}}^{e}\right)\right)\left(\sigma\left(c_{b_{e}}^{e}\right)-x_{e}^{0}\right)}
$$

and $\omega_{\sigma, b}^{e}\left(x_{e}^{0}\right)=1$ if $b_{e}=0$. Then the weight function is given by the formula

$$
\omega\left(x^{0}, t, \boldsymbol{k}\right)=\sum_{b \in B} \sum_{\sigma \in \Sigma(b)} \omega_{\sigma, b}\left(x^{0}, t\right) F_{d_{\sigma, b}} v .
$$

The weight function is a function of $t$ depending on parameters $x^{0}$.

Lemma 11.1 (Lemma 2.1 in [16]). The weight function is regular on $U$. 


\subsection{Bethe vectors}

Theorem 11.1 ([2, 3, 19]). Let $\mathfrak{g}$ be a simple Lie algebra. Let $\boldsymbol{\Lambda}=\left(\Lambda_{1}, \ldots, \Lambda_{N}\right), \Lambda_{b} \in \mathfrak{h}^{*}$, be a collection of weights, $\boldsymbol{k}=\left(k_{1}, \ldots, k_{r}\right)$ a collection of nonnegative integers. Assume that $x^{0} \in \mathbb{C}^{N}$ has distinct coordinates. Assume that $p \in \mathbb{C}^{k}$ is a critical point of the master function $\Phi\left(x^{0}, \cdot, \boldsymbol{\Lambda}, \boldsymbol{k}\right): U \rightarrow U$. Then the vector $\omega\left(x^{0}, p, \boldsymbol{k}\right)$ (if nonzero) belongs to $\operatorname{Sing} V_{\boldsymbol{\Lambda}}\left[\Lambda_{\infty}\right]$ and is an eigenvector of the Gaudin Hamiltonians $\boldsymbol{K}_{1}\left(x^{0}\right), \ldots, \boldsymbol{K}_{N}\left(x^{0}\right)$.

The theorem also follows directly from Theorem 6.16.2 in [23], cf. Theorem 7.2.5 in [23], see also Theorem 4.2.2 in [6].

The vector $\omega\left(x^{0}, p, \boldsymbol{k}\right)$ is called the Bethe vector corresponding to the critical point $p$.

Theorem 11.2. If $p$ is an isolated critical point of the master function, then

$$
S_{\boldsymbol{\Lambda}}\left(\omega\left(x^{0}, p, \boldsymbol{k}\right), \omega\left(x^{0}, p, \boldsymbol{k}\right)\right)=\operatorname{det}\left(\frac{\partial^{2} \Phi}{\partial t_{j}^{(i)} \partial t_{j^{\prime}}^{\left(i^{\prime}\right)}}\right)\left(x^{0}, p, \boldsymbol{\Lambda}, \boldsymbol{k}\right) .
$$

In particular, if the critical point is nondegenerate, then the Bethe vector is nonzero.

This theorem is proved for $\mathfrak{g}=\mathfrak{s l}_{r+1}$ in [16] and for any simple Lie algebra in [27].

\subsection{Identification of Gaudin and naive geometric Hamiltonians}

Let us identify constructions and statements of Theorems 11.1, 11.2 and of Theorem 10.14. First of all let us define the discriminantal arrangement associated with the Gaudin model in Theorems 11.2, 11.1.

The Gaudin model is determined by a simple Lie algebra $\mathfrak{g}$, a nondegenerate $\mathfrak{g}$-invariant bilinear form $($,$) , simple roots \alpha_{1}, \ldots, \alpha_{r}$, highest weights $\Lambda_{1}, \ldots, \Lambda_{N}$, distinct complex numbers $x_{1}^{0}, \ldots, x_{N}^{0}$ and a vector $\boldsymbol{k}=\left(k_{1}, \ldots, k_{r}\right)$ of nonnegative integers.

Let us take these $\mathfrak{h}^{*}, \alpha_{1}, \ldots, \alpha_{r}, \Lambda_{1}, \ldots, \Lambda_{N},(),, \boldsymbol{k}=\left(k_{1}, \ldots, k_{r}\right)$ as Data 10.1 to define a family of prediscriminantal arrangements. Let us choose a point $z^{0} \in U(X)$ by conditions $x_{b}\left(z^{0}\right)=x_{b}^{0}$ for $b=1, \ldots, N$. The corresponding weighted discriminantal arrangement $\left(\mathbb{A}\left(z^{0}\right), a\right)$ of Section 10.2 is exactly the weighted arrangement defined by the master function in (11.1). In particular, we have $\Phi\left(x^{0}, \cdot, \boldsymbol{\Lambda}, \boldsymbol{k}\right)=\Phi\left(z^{0}, \cdot\right)$ where $\Phi\left(z^{0}, \cdot\right)$ is the master function of the arrangement $\left(\mathbb{A}\left(z^{0}\right), a\right)$.

In [23] an isomorphism $\gamma: W^{-}\left(z^{0}\right) \rightarrow V_{\boldsymbol{\Lambda}}\left[\Lambda_{\infty}\right]$ was constructed with the following properties $(i)-(i v)$.

(i) The isomorphism $\gamma$ identifies Sing $W^{-}\left(z^{0}\right)$ with $\operatorname{Sing} V_{\boldsymbol{\Lambda}}\left[\Lambda_{\infty}\right]$.

(ii) The isomorphism $\gamma$ identifies a naive geometric Hamiltonian $\left.K_{\partial_{x_{b}}}^{*}\right|_{\operatorname{Sing} W^{-}\left(z^{0}\right)}$ with the Gaudin Hamiltonian $\left.\boldsymbol{K}_{b}\left(x^{0}\right)\right|_{\operatorname{Sing} V_{\boldsymbol{\Lambda}}\left[\Lambda_{\infty}\right]}$ up to addition of a scalar operator. More precisely, for any $b=1, \ldots, N$, there is a number $c_{b}$ such that

$$
\left.\left.\gamma\right|_{\operatorname{Sing} W^{-}\left(z^{0}\right)}\left(K_{\partial_{x_{b}}}^{*}+c_{b}\right)\right|_{\operatorname{Sing} W^{-}\left(z^{0}\right)}=\left.\left.\boldsymbol{K}_{b}\left(x^{0}\right)\right|_{\operatorname{Sing} V_{\boldsymbol{\Lambda}}\left[\Lambda_{\infty}\right]} \gamma\right|_{\operatorname{Sing} W^{-}\left(z^{0}\right)} \cdot
$$

(iii) The isomorphism $\gamma$ identifies the contravariant form on $W^{-}\left(z^{0}\right)$ (multiplied by $\left.(-1)^{k} k_{1} ! \cdots k_{r} !\right)$ with the tensor Shapovalov form on $V_{\boldsymbol{\Lambda}}\left[\Lambda_{\infty}\right]$,

$$
S_{\boldsymbol{\Lambda}}(\gamma(x), \gamma(y))=(-1)^{k} k_{1} ! \cdots k_{r} ! S^{(a)}(x, y) \quad \text { for any } x, y \in V_{\boldsymbol{\Lambda}}\left[\Lambda_{\infty}\right]
$$


(iv) Let $\boldsymbol{E} \in \mathbb{C}(t)_{U\left(\mathbb{A}\left(z^{0}\right)\right)} \otimes W\left(z^{0}\right)$ be the canonical element of the arrangement $\mathbb{A}\left(z^{0}\right)$. Let Ant $=\sum_{\sigma \in S_{k}}(-1)^{\sigma} \sigma: W\left(z^{0}\right) \rightarrow W^{-}\left(z^{0}\right)$ be the anti-symmetrization operator. Consider the element $(1 \otimes$ Ant $) \boldsymbol{E} \in \mathbb{C}(t)_{U\left(\mathbb{A}\left(z^{0}\right)\right)} \otimes W^{-}\left(z^{0}\right)$. Let $\omega\left(x^{0}, \cdot, \boldsymbol{k}\right) \in \mathbb{C}(t)_{U\left(\mathbb{A}\left(z^{0}\right)\right)} \otimes V_{\boldsymbol{\Lambda}}\left[\Lambda_{\infty}\right]$ be the weight function defined by (11.2). Then

$$
(1 \otimes \gamma)(1 \otimes \mathrm{Ant}) \boldsymbol{E}=k_{1} ! \cdots k_{r} ! \omega
$$

See Theorems 5.13, 6.16.2, 6.6 and 7.2.5 in [23], see also Section 5.5 in [27].

Now Theorem 11.2 follows from items $(i v)$ and $(v i)$ of Theorem 10.14 and Theorem 11.1 follows from Lemma 10.3 and Theorem 2.8.

From statements $(i)-(i v)$ and Theorem 10.14 we get the following improvement of Theorem 11.2.

Theorem 11.3. For any simple Lie algebra $\mathfrak{g}$, if $p$ is an isolated critical point of the master function $\Phi\left(x^{0}, \cdot, \boldsymbol{\Lambda}, \boldsymbol{k}\right)$, then the corresponding Bethe vector $\omega\left(x^{0}, p, \boldsymbol{k}\right)$ is nonzero.

\subsection{Bethe algebra}

The subalgebra of End(Sing $\left.V_{\boldsymbol{\Lambda}}[\mu]\right)$ generated by the Gaudin Hamiltonians can be extended to a larger commutative subalgebra called the Bethe algebra. A construction of the Bethe algebra for any simple Lie algebra $\mathfrak{g}$ is given in [4]. As a result of that construction, for any $x^{0}$ one obtains a commutative subalgebra $B\left(x^{0}\right) \subset(U \mathfrak{g})^{\otimes N}$ which commutes with the diagonal subalgebra $U \mathfrak{g} \subset(U \mathfrak{g})^{\otimes N}$. To define the Bethe algebra of $V_{\boldsymbol{\Lambda}}$ or of Sing $V_{\boldsymbol{\Lambda}}[\mu]$ one considers the image of $B\left(x^{0}\right)$ in $\operatorname{End}\left(V_{\boldsymbol{\Lambda}}\right)$ or in $\operatorname{End}\left(\operatorname{Sing} V_{\boldsymbol{\Lambda}}[\mu]\right)$. The Gaudin Hamiltonians $\boldsymbol{K}_{b}\left(x^{0}\right)$ are elements of the Bethe algebra of $V_{\boldsymbol{\Lambda}}$ or of Sing $V_{\boldsymbol{\Lambda}}\left[\Lambda_{\infty}\right]$.

A more straightforward construction of the Bethe algebra is known for the Gaudin model of $\mathfrak{g l}_{r+1}$, see [24]. Below we give its description.

Let $e_{i j}, i, j=1, \ldots, r+1$, be the standard generators of $\mathfrak{g l}_{r+1}$ satisfying the relations $\left[e_{i j}, e_{s k}\right]=$ $\delta_{j s} e_{i k}-\delta_{i k} e_{s j}$. Let $\mathfrak{h} \subset \mathfrak{g l}_{r+1}$ be the Cartan subalgebra generated by $e_{i i}, i=1, \ldots, r+1$. Let $\mathfrak{h}^{*}$ be the dual space. Let $\epsilon_{i}, i=1, \ldots, r+1$, be the basis of $\mathfrak{h}^{*}$ dual to the basis $e_{i i}, i=1, \ldots, r+1$, of $\mathfrak{h}$. Let $\alpha_{1}, \ldots, \alpha_{r} \in \mathfrak{h}^{*}$ be simple roots, $\alpha_{i}=\epsilon_{i}-\epsilon_{i+1}$. Let (, ) be the standard scalar product on $\mathfrak{h}^{*}$ such that the basis $\epsilon_{i}, i=1, \ldots, r+1$, is orthonormal.

Let $\mathfrak{g l}_{r+1}[s]=\mathfrak{g l}_{r+1} \otimes \mathbb{C}[s]$ be the Lie algebra of $\mathfrak{g l}_{r+1}$-valued polynomials with the pointwise commutator. For $g \in \mathfrak{g l}_{r+1}$, we set $g(u)=\sum_{i=0}^{\infty}\left(g \otimes s^{i}\right) u^{-i-1}$. We identify $\mathfrak{g l}_{r+1}$ with the subalgebra $\mathfrak{g l}_{r+1} \otimes 1$ of constant polynomials in $\mathfrak{g l}_{r+1}[s]$. Hence, any $\mathfrak{g l}_{r+1}[s]$-module has a canonical structure of a $\mathfrak{g l}_{r+1}$-module.

For each $a \in \mathbb{C}$, there exists an automorphism $\rho_{a}$ of $\mathfrak{g l}_{r+1}[s], \rho_{a}: g(u) \mapsto g(u-a)$. Given a $\mathfrak{g l}_{r+1}[s]$-module $W$, we denote by $W(a)$ the pull-back of $W$ through the automorphism $\rho_{a}$. As $\mathfrak{g l}_{r+1}$-modules, $W$ and $W(a)$ are isomorphic by the identity map.

We have the evaluation homomorphism, $\mathfrak{g l}_{r+1}[s] \rightarrow \mathfrak{g l}_{r+1}, g(u) \mapsto g u^{-1}$. Its restriction to the subalgebra $\mathfrak{g l}_{r+1} \subset \mathfrak{g l}_{r+1}[s]$ is the identity map. For any $\mathfrak{g l}_{r+1}$-module $W$, we denote by the same letter the $\mathfrak{g l}_{r+1}[s]$-module, obtained by pulling $W$ back through the evaluation homomorphism.

Given an algebra $A$ and an $(r+1) \times(r+1)$-matrix $C=\left(c_{i j}\right)$ with entries in $A$, we define its row determinant to be

$$
\operatorname{rdet} C=\sum_{\sigma \in \Sigma_{r+1}}(-1)^{\sigma} c_{1 \sigma(1)} c_{2 \sigma(2)} \cdots c_{r+1 \sigma(r+1)}
$$


Define the universal differential operator $\mathfrak{D}_{B}$ by the formula

$$
\mathfrak{D}_{B}=\operatorname{rdet}\left(\begin{array}{cccc}
\partial_{u}-e_{11}(u) & -e_{21}(u) & \ldots & -e_{r+11}(u) \\
-e_{12}(u) & \partial_{u}-e_{22}(u) & \ldots & -e_{r+12}(u) \\
\ldots & \ldots & \ldots & \ldots \\
-e_{1 r+1}(u) & -e_{2 r+1}(u) & \ldots & \partial_{u}-e_{r+1 r+1}(u)
\end{array}\right) .
$$

We have

$$
\mathfrak{D}_{B}=\partial_{u}^{r+1}+\sum_{i=1}^{r+1} B_{i} \partial_{u}^{r+1-i}, \quad B_{i}=\sum_{j=i}^{\infty} B_{i j} u^{-j}, \quad B_{i j} \in U \mathfrak{g l}_{r+1}[s] .
$$

The unital subalgebra of $U \mathfrak{g l}_{r+1}[s]$ generated by $B_{i j}, i=1, \ldots, r+1, j \geqslant i$, is called the Bethe algebra and denoted by $B$.

By [24], cf. [10], the algebra $B$ is commutative, and $B$ commutes with the subalgebra $U \mathfrak{g l}_{r+1} \subset$ $U \mathfrak{g l}_{r+1}[s]$.

As a subalgebra of $U \mathfrak{g l}_{r+1}[s]$, the algebra $B$ acts on any $\mathfrak{g l}_{r+1}[s]$-module $W$. Since $B$ commutes with $U \mathfrak{g l}_{r+1}$, it preserves the $\mathfrak{g l}_{r+1}$ weight subspaces of $W$ and the subspace Sing $W$ of $\mathfrak{g l}_{r+1^{-}}$ singular vectors.

If $W$ is a $B$-module, then the image of $B$ in $\operatorname{End}(W)$ is called the Bethe algebra of $W$.

Let $V_{\boldsymbol{\Lambda}}=\otimes_{b=1}^{n} V_{\Lambda_{b}}$ be a tensor product of irreducible highest weight $\mathfrak{g l}_{r+1}$-modules. For given $x^{0}=\left(x_{1}^{0}, \ldots, x_{N}^{0}\right)$, consider $V_{\boldsymbol{\Lambda}}$ as the $\mathfrak{g l}_{r+1}[s]$-module $\otimes_{b=1}^{n} V_{\Lambda_{b}}\left(x_{b}^{0}\right)$. This $\mathfrak{g l}_{r+1}[s]$-module structure on $V_{\boldsymbol{\Lambda}}$ provides $V_{\boldsymbol{\Lambda}}$ with a Bethe algebra, a commutative subalgebra of $\operatorname{End}\left(V_{\boldsymbol{\Lambda}}\right)$. It is known that this Bethe algebra of $V_{\boldsymbol{\Lambda}}$ contains the Gaudin Hamiltonians $\boldsymbol{K}_{b}\left(x^{0}\right), b=1, \ldots, N$. In fact, the Gaudin Hamiltonians are suitably normalized residues of the generating function $B_{2}(u)$, see Appendix B in [10].

Theorem 11.4 ([12]). Consider $V_{\boldsymbol{\Lambda}}$ as the $\mathfrak{g l}_{r+1}[s]$-module $\otimes_{b=1}^{n} V_{\Lambda_{b}}\left(x_{b}^{0}\right)$. Then any element $B \in B$ acts on $V_{\boldsymbol{\Lambda}}$ as a symmetric operator with respect to the tensor Shapovalov form, $S_{\boldsymbol{\Lambda}}(B u, v)=S_{\boldsymbol{\Lambda}}(u, B v)$ for any $u, v \in V_{\boldsymbol{\Lambda}}$.

\section{6 $\mathfrak{g l}_{r+1}$ Bethe algebra and critical points of the master function}

In [15] the following generalization of Theorem 11.1 for $\mathfrak{g}=\mathfrak{g l}_{r+1}$ was obtained.

Let $\mathfrak{g}=\mathfrak{g l}_{r+1}$. A sequence of integers $\Lambda=\left(\lambda_{1}, \ldots, \lambda_{r+1}\right)$ such that $\lambda_{1} \geqslant \lambda_{2} \geqslant \cdots \geqslant$ $\lambda_{r+1} \geqslant 0$ is called a partition with at most $r+1$ parts. We identify partitions $\Lambda$ with vectors $\lambda_{1} \epsilon_{1}+\cdots+\lambda_{r+1} \epsilon_{r+1}$ of $\mathfrak{h}^{*}$.

Let $\boldsymbol{\Lambda}=\left(\Lambda_{1}, \ldots, \Lambda_{N}\right)$ be a collection of partitions, where $\Lambda_{b}=\left(\lambda_{b, 1}, \ldots, \lambda_{b, r+1}\right)$ and $\lambda_{b, r+1}=0$. Let $\boldsymbol{k}=\left(k_{1}, \ldots, k_{r}\right)$ be nonnegative integers such that

$$
\Lambda_{\infty}=\sum_{b=1}^{N} \Lambda_{b}-\sum_{i=1}^{r} k_{i} \alpha_{i}
$$

is a partition. We consider the $\mathfrak{g l}_{r+1}$ Gaudin model with parameters $x^{0}=\left(x_{1}^{0}, \ldots, x_{N}^{0}\right)$ on Sing $V_{\boldsymbol{\Lambda}}\left[\Lambda_{\infty}\right]$ where $V_{\boldsymbol{\Lambda}}=V_{\Lambda_{1}} \otimes \cdots \otimes V_{\Lambda_{N}}$.

Consider the master function $\Phi\left(x^{0}, t, \boldsymbol{\Lambda}, \boldsymbol{k}\right)$ defined by (11.1) and the weight function $\omega\left(x^{0}, t, \boldsymbol{k}\right)$ defined by (11.2).

Let $u$ be a variable. Define polynomials $T_{1}, \ldots, T_{r} \in \mathbb{C}[u], Q_{1}, \ldots, Q_{r} \in \mathbb{C}[u, t]$,

$$
T_{i}(u)=\prod_{b=1}^{N}\left(u-x_{b}\right)^{\left(\Lambda_{b}, \alpha_{i}\right)}, \quad Q_{i}(u, t)=\prod_{j=1}^{k_{i}}\left(u-t_{j}^{(i)}\right),
$$


and the differential operator

$$
\begin{aligned}
\mathfrak{D}_{\Phi}= & \left(\partial_{u}-\log ^{\prime}\left(\frac{T_{1} \ldots T_{r}}{Q_{1}}\right)\right) \\
& \times\left(\partial_{u}-\log ^{\prime}\left(\frac{Q_{1} T_{2} \cdots T_{r}}{Q_{2}}\right)\right) \cdots\left(\partial_{u}-\log ^{\prime}\left(\frac{Q_{r-1} T_{r}}{Q_{r}}\right)\right)\left(\partial_{u}-\log ^{\prime}\left(Q_{r}\right)\right),
\end{aligned}
$$

where $\partial_{u}=d / d u$ and $\log ^{\prime} f$ denotes $(d f / d u) / f$. We have

$$
\mathfrak{D}_{\Phi}=\partial_{u}^{N+1}+\sum_{i=1}^{N+1} G_{i} \partial_{u}^{N+1-i}, \quad G_{i}=\sum_{j=i}^{\infty} G_{i j} u^{-j},
$$

where $G_{i j} \in \mathbb{C}[\boldsymbol{t}]$.

Let $p \in U$ be an isolated critical point of the master function $\Phi(x,, \cdot, \boldsymbol{\Lambda}, \boldsymbol{k})$ with Milnor number $\mu$. Let $A_{p, \Phi}$ be its local algebra. For $f \in \mathbb{C}(t)_{U}$ denote by $[f]$ the image of $f$ in $A_{p, \Phi}$. Denote

$$
\left[\mathfrak{D}_{\Phi}\right]=\partial_{u}^{N+1}+\sum_{i=1}^{N+1}\left[G_{i}\right] \partial_{u}^{N+1-i}
$$

where $\left[G_{i}\right]=\sum_{j=i}^{\infty}\left[G_{i j}\right] u^{-j}$. Let $[\omega] \in A_{p, \Phi} \otimes V_{\Lambda}\left[\Lambda_{\infty}\right]$ be the element induced by the weight function. The element $[\omega]$ belongs to $A_{p, \Phi} \otimes \operatorname{Sing} V_{\boldsymbol{\Lambda}}\left[\Lambda_{\infty}\right]$, see [23], and we have $S_{\boldsymbol{\Lambda}}([\omega],[\omega])=\left[\operatorname{Hess}_{t} \Phi\right]$, see $[16,27]$.

Theorem $11.5([10])$. For any $i=1, \ldots, r+1, j \geqslant i$, we have

$$
\left(1 \otimes B_{i j}\right)[\omega]=\left(\left[G_{i j}\right]_{p} \otimes 1\right)[\omega]
$$

in $A_{p, \Phi} \otimes \operatorname{Sing} V_{\boldsymbol{\Lambda}}\left[\Lambda_{\infty}\right]$.

This statement is the Bethe ansatz method to construct eigenvectors of the Bethe algebra in the $\mathfrak{g l}_{r+1}$ Gaudin model starting with a critical point of the master function.

Let $g_{1}, \ldots, g_{\mu}$ be a basis of $A_{p, \Phi}$ considered as a $\mathbb{C}$-vector space. Write $[\omega]_{p}=\sum_{i} g_{i} \otimes w_{i}$, with $w_{i} \in \operatorname{Sing} V_{\boldsymbol{\Lambda}}\left[\Lambda_{\infty}\right]$. Denote by $\mathcal{Y}_{p} \subset \operatorname{Sing} V_{\boldsymbol{\Lambda}}\left[\Lambda_{\infty}\right]$ the vector subspace spanned by $w_{1}, \ldots, w_{\mu}$. Let $(,)_{p}$ be the bilinear form on $A_{p, \Phi}$. Define a linear map

$$
\alpha_{p}: A_{p, \Phi} \rightarrow \mathcal{Y}_{p}, \quad f \mapsto\left(f,[\omega]_{p}\right)_{p}=\sum_{i=1}^{\mu}\left(f, g_{i}\right)_{p} w_{i} .
$$

Theorem $11.6([15])$.

(i) The subspace $\mathcal{Y}_{p} \subset \operatorname{Sing} V_{\boldsymbol{\Lambda}}\left[\Lambda_{\infty}\right]$ is a B-submodule. Let $A \mathcal{Y}_{p} \subset$ End $\left(\mathcal{Y}_{p}\right)$ be the Bethe algebra of $\mathcal{Y}_{p}$. Denote by $\bar{B}_{i j}$ the image in $A_{\mathcal{Y}_{p}}$ of generators $B_{i j} \in B$.

(ii) The map $\alpha_{p}: A_{p, \Phi} \rightarrow \mathcal{Y}_{p}$ is an isomorphism of vector spaces.

(iii) The map $\left[G_{i j}\right]_{p} \mapsto \bar{B}_{i j}$ extends uniquely to an algebra isomorphism $\beta_{p}: A_{p, \Phi} \rightarrow A_{\mathcal{Y}_{p}}$.

(iv) The isomorphisms $\alpha_{p}$ and $\beta_{p}$ identify the regular representation of $A_{p, \Phi}$ and the $B$-module $\mathcal{Y}_{p}$, that is, for any $f, g \in A_{p, \Phi}$ we have $\alpha_{p}(f g)=\beta_{p}(f) \alpha_{p}(g)$.

(v) The value of the weight function at $p$ is a nonzero vector of $\operatorname{Sing} V_{\boldsymbol{\Lambda}}\left[\Lambda_{\infty}\right]$.

(vi) Let $p_{1}, \ldots, p_{d}$ be a list of isolated critical points of $\Phi\left(x^{0}, \cdot, \boldsymbol{\Lambda}, \boldsymbol{k}\right)$ such that the orbits $O\left(p_{1}\right), \ldots, O\left(p_{d}\right)$ do not intersect. Let $\mathcal{Y}_{p_{s}}=\alpha_{p_{s}}\left(A_{p_{s}, \Phi}\right) \subset \operatorname{Sing} V_{\boldsymbol{\Lambda}}\left[\Lambda_{\infty}\right], s=1, \ldots, d$, be the corresponding subspaces. Then the sum of these subspaces is direct and orthogonal. 
(vii) Denote $\mathcal{Y}=\oplus_{s=1}^{d} \mathcal{Y}_{p_{s}}$. Denote $A \mathcal{Y}$ the Bethe algebra of $\mathcal{Y}$. Consider the isomorphisms

$$
\begin{aligned}
& \alpha=\oplus_{s=1}^{d} \alpha_{p_{s}}: \oplus_{s=1}^{d} A_{p_{s}, \Phi} \rightarrow \oplus_{s=1}^{d} \mathcal{Y}_{p_{s}}, \\
& \beta=\oplus_{s=1}^{d} \beta_{p_{s}}: \oplus_{s=1}^{d} A_{p_{s}, \Phi} \rightarrow \oplus_{s=1}^{d} A_{Y_{p_{s}}} .
\end{aligned}
$$

Then

(a) $A \mathcal{Y}=\oplus_{s=1}^{d} A \mathcal{Y}_{p_{s}}$;

(b) $A_{\mathcal{Y}}$ is a maximal commutative subalgebra of $\operatorname{End}(\mathcal{Y})$;

(c) the isomorphisms $\alpha, \beta$ identify the regular representation of the algebra $\oplus_{s=1}^{d} A_{p_{s}, \Phi}$ and the $A_{\mathcal{Y}}$-module $\mathcal{Y}$.

Let us identify constructions and statements of Theorem 11.6 and of Theorems 10.14, 10.17 .

Consider the discriminantal arrangement $\mathbb{A}\left(z^{0}\right), z^{0} \in U(X)$, defined in Section 11.4 and the isomorphism $\left.\gamma\right|_{\operatorname{Sing} W^{-}\left(z^{0}\right)}: \operatorname{Sing} W^{-}\left(z^{0}\right) \rightarrow \operatorname{Sing} V_{\boldsymbol{\Lambda}}\left[\Lambda_{\infty}\right]$ of that section. According to statements $(i)-(i v)$ of Section 11.4, we have $\gamma\left(Y_{O\left(p_{s}\right)}^{-}\right)=\mathcal{Y}_{p_{s}}$, where $Y_{O\left(p_{s}\right)}^{-} \subset \operatorname{Sing} W^{-}\left(z^{0}\right)$ is the subspace in Theorems 10.14, 10.17 and $\mathcal{Y}_{p_{s}} \subset \operatorname{Sing} V_{\boldsymbol{\Lambda}}\left[\Lambda_{\infty}\right]$ is the subspace in Theorem 11.6. Moreover, $\gamma$ identifies the algebra $A_{Y_{O\left(p_{s}\right)}^{-}}$of geometric Hamiltonians on $Y_{O\left(p_{s}\right)}^{-}$with the Bethe algebra of $\mathcal{Y}_{p_{s}}$.

Additional information that is given by Theorems 10.14, 10.17 is the following theorem.

Theorem 11.7. The monomorphism $\alpha_{p}$ of Theorem 11.6 identifies the tensor Shapovalov form on $\mathcal{Y}_{p}$ and the residue bilinear form on $A_{p, \Phi}$.

Proof. The theorem is a corollary of properties (iii), (iv) in Section 11.4 and Lemma 10.12.

\subsection{Expectations}

One may expect that for the Gaudin models of any simple Lie algebras the associated Bethe algebra defined in [4] coincides with the algebra of geometric Hamiltonians associated with the arrangement of the corresponding master function. That topic will be discussed in a forthcoming paper.

Below we consider three examples in which we have a well-defined algebra of geometric Hamiltonians on Sing $V_{\boldsymbol{\Lambda}}\left[\Lambda_{\infty}\right]$.

\subsubsection{Example}

Consider the Gaudin model corresponding to the following data: the Lie algebra $\mathfrak{g l}_{r+1}$, the collection of dominant integral weights $\boldsymbol{\Lambda}=\left(\Lambda_{1}, \ldots, \Lambda_{N}\right)$ with $\Lambda_{b}=(1,0, \ldots, 0)$ for all $b$, a vector of nonnegative integers $\boldsymbol{k}=\left(k_{1}, \ldots, k_{r}\right)$ such that $\Lambda_{\infty}$ is a partition, a collection of generic distinct complex numbers $x^{0}=\left(x_{1}^{0}, \ldots, x_{N}^{0}\right)$.

By [16], for generic $x^{0}$ the associated master function has a collection of critical points $p_{1}, \ldots, p_{d}$ such that the $S_{\boldsymbol{k}}$-orbits of these points do not intersect and the sum of Milnor numbers of these points equals the dimension of Sing $V_{\boldsymbol{\Lambda}}\left[\Lambda_{\infty}\right]$. In this case Theorem 10.17 defines a maximal commutative subalgebra $A_{\operatorname{Sing}\left(W^{-}\left(z^{0}\right)\right)} \subset \operatorname{End}\left(\operatorname{Sing}\left(W^{-}\left(z^{0}\right)\right)\right)$ containing naive geometric Hamiltonians. The isomorphism $\gamma$ sends $A_{\text {Sing }\left(W^{-}\left(z^{0}\right)\right)}$ to a maximal commutative subalgebra of End(Sing $\left.V_{\boldsymbol{\Lambda}}\left[\Lambda_{\infty}\right]\right)$ containing the Gaudin Hamiltonians. By [12, 13], the Bethe algebra of Sing $V_{\boldsymbol{\Lambda}}\left[\Lambda_{\infty}\right]$ is a maximal commutative subalgebra of $\operatorname{End}\left(\operatorname{Sing} V_{\boldsymbol{\Lambda}}\left[\Lambda_{\infty}\right]\right)$ and the Bethe algebra of Sing $V_{\boldsymbol{\Lambda}}\left[\Lambda_{\infty}\right]$ is generated by the Gaudin Hamiltonians. Hence, in this case, $\gamma$ establishes an isomorphism of the algebra of geometric Hamiltonians $A_{\operatorname{Sing}\left(W^{-}\left(z^{0}\right)\right)}$ and the Bethe algebra of Sing $V_{\boldsymbol{\Lambda}}\left[\Lambda_{\infty}\right]$. 


\subsubsection{Example}

Consider the Gaudin model corresponding to the following data: the Lie algebra $\mathfrak{g l}_{2}$, a collection of weights $\boldsymbol{\Lambda}=\left(\Lambda_{1}, \ldots, \Lambda_{N}\right)$ with $\Lambda_{b}=\lambda_{b} \alpha_{1}$ such that $\lambda_{b} \in \mathbb{R}_{<0}$ for all $b$, a nonnegative integer $\boldsymbol{k}=\left(k_{1}\right)$, a collection of distinct complex numbers $x^{0}=\left(x_{1}^{0}, \ldots, x_{N}^{0}\right)$.

Let $\left(\mathbb{A}\left(z^{0}\right), a\right)$ be the associated discriminantal arrangement defined in Section 11.4. By our assumptions, the weights $a$ of the discriminant arrangement are all positive. Corollary 10.18 defines in this case a maximal commutative subalgebra of $\operatorname{End}\left(\operatorname{Sing} W^{-}\left(z^{0}\right)\right)$ ) and the isomorphism $\gamma$ sends this subalgebra to a maximal commutative subalgebra of $\operatorname{End}\left(\operatorname{Sing} V_{\boldsymbol{\Lambda}}\left[\Lambda_{\infty}\right]\right)$, which contains Gaudin Hamiltonians. One may expect that this commutative subalgebra of $\operatorname{End}\left(\operatorname{Sing} V_{\boldsymbol{\Lambda}}\left[\Lambda_{\infty}\right]\right)$ coincides with the Bethe algebra of Sing $V_{\boldsymbol{\Lambda}}\left[\Lambda_{\infty}\right]$.

\subsubsection{Example}

Consider the Gaudin model corresponding to the following data: a simple Lie algebra $\mathfrak{g}$, a collection of dominant integral weights $\boldsymbol{\Lambda}=\left(\Lambda_{1}, \ldots, \Lambda_{N}\right)$, a vector of nonnegative integers $\boldsymbol{k}=$ $\left(k_{1}, \ldots, k_{r}\right)$ with $k_{i} \leqslant 1$ for all $i$, a collection of distinct complex numbers $x^{0}=\left(x_{1}^{0}, \ldots, x_{N}^{0}\right)$.

Let $\left(\mathbb{A}\left(z^{0}\right), a\right)$ be the associated discriminantal arrangement. By our assumptions, the weights $a$ of the discriminant arrangement are all negative and the group $S_{\boldsymbol{k}}$ is trivial. In this case the isomorphism $\gamma$ identifies $\operatorname{Sing} \mathcal{F}^{k}\left(\mathbb{A}\left(z^{0}\right)\right)$ and the space $\operatorname{Sing} V_{\boldsymbol{\Lambda}}\left[\Lambda_{\infty}\right]$. Theorem 9.17 defines in this case a maximal commutative subalgebra of $\operatorname{End}\left(\operatorname{Sing} \mathcal{F}^{k}\left(\mathbb{A}\left(z^{0}\right)\right)\right)$ and the isomorphism $\gamma$ sends this subalgebra to a maximal commutative subalgebra of $\operatorname{End}\left(\operatorname{Sing} V_{\boldsymbol{\Lambda}}\left[\Lambda_{\infty}\right]\right)$, which contains the Gaudin Hamiltonians. One may expect that this commutative subalgebra of $\operatorname{End}\left(\operatorname{Sing} V_{\boldsymbol{\Lambda}}\left[\Lambda_{\infty}\right]\right)$ coincides with the Bethe algebra of Sing $V_{\boldsymbol{\Lambda}}\left[\Lambda_{\infty}\right]$.

\section{Acknowledgments}

The idea that an analog of the Bethe ansatz construction does exist for an arbitrary arrangement of hyperplanes was formulated long time ago in [26]. That program had been realized partially in [27].

This paper is an extended exposition of my lectures at Mathematical Society of Japan Seasonal Institute on Arrangements of Hyperplanes in August of 2009. I thank organizers for invitation and Hokkaido University for hospitality. I thank for hospitality Université Paul Sabatier in Toulouse, where this paper had been finished. I thank E. Mukhin, V. Schechtman, V. Tarasov, H. Terao for discussions.

The author was supported in part by NSF grant DMS-0555327.

\section{References}

[1] Arnold V.I., Gusein-Zade S.M., Varchenko A.M., Singularities of differentiable maps, Vol. II, Monodromy and asymptotics of integrals, Monographs in Mathematics, Vol. 83, Birkhäuser, Boston, MA, 1988.

[2] Babujian H.M., Off-shell Bethe ansatz equations and $N$-point correlators in the SU(2) WZNW theory, J. Phys. A: Math. Gen. 26 (1993), 6981-6990.

[3] Babujian H.M., Flume R., Off-shell Bethe ansatz equation for Gaudin magnets and solutions of KnizhnikZamolodchikov equations, Modern Phys. Lett. A 9 (1994), 2029-2039, hep-th/9310110.

[4] Feigin B., Frenkel E., Reshetikhin N., Gaudin model, Bethe ansatz and critical level, Comm. Math. Phys. 166 (1994), 29-62, hep-th/9402022.

[5] Feigin B., Frenkel E., Rybnikov L., Opers with irregular singularity and spectra of the shift of argument subalgebra, Duke Math. J. 155 (2010), 337-363, arXiv:0712.1183.

[6] Feigin B., Schechtman V., Varchenko A., On algebraic equations satisfied by hypergeometric correlators in WZW models. II, Comm. Math. Phys. 170 (1995), 219-247, hep-th/9407010. 
[7] Gaudin M., Diagonalisation d'une classe d'Hamiltoniens de spin, J. Physique 37 (1976), 1087-1098.

[8] Gaudin M., La fonction d'onde de Bethe, Collection du Commissariat l'Érgie Atomique, Série Scientifique, Masson, Paris, 1983.

[9] Griffiths Ph., Harris J., Principles of algebraic geometry, John Wiley \& Sons, Inc., New York, 1994.

[10] Mukhin E., Tarasov V., Varchenko A., Bethe eigenvectors of higher transfer matrices, J. Stat. Mech. Theory Exp. 2006 (2006), no. 8, P08002, 44 pages, math.QA/0605015.

[11] Mukhin E., Tarasov V., Varchenko A., The B. and M. Shapiro conjecture in real algebraic geometry and the Bethe ansatz, Ann. of Math. (2) 170 (2009), 863-881, math.AG/0512299.

[12] Mukhin E., Tarasov V., Varchenko A., Schubert calculus and representations of the general linear group, J. Amer. Math. Soc. 22 (2009), 909-940, arXiv:0711.4079.

[13] Mukhin E., Tarasov V., Varchenko A., Gaudin Hamiltonians generate the Bethe algebra of a tensor power of vector representation of $\mathfrak{g l}_{N}$, Algebra $i$ Analiz 22 (2010), no. 3, 177-190, arXiv:0904.2131.

[14] Mukhin E., Tarasov V., Varchenko A., Three sides of the geometric Langlands correspondence for $\mathfrak{g l}_{N}$ Gaudin model and Bethe vector averaging maps, arXiv:0907.3266.

[15] Mukhin E., Tarasov V., Varchenko A., Bethe algebra of the $\mathfrak{g l}_{N+1}$ Gaudin model and algebra of functions on the critical set of the master function, arXiv:0910.4690.

[16] Mukhin E., Varchenko A., Norm of a bethe vector and the Hessian of the master function, Compos. Math. 141 (2005), 1012-1028, math.QA/0402349.

[17] Orlik P., Terao H., The number of critical points of a product of powers of linear functions, Invent. Math. 120 (1995), 1-14.

[18] Orlik P., Terao H., Arrangements and hypergeometric integrals, MSJ Memoirs, Vol. 9, Mathematical Society of Japan, Tokyo, 2001.

[19] Reshetikhin N., Varchenko A., Quasiclassical asymptotics of solutions to the KZ equations, in Geometry, Topology, \& Physics, Conf. Proc. Lecture Notes Geom. Topology, Vol. 4, Int. Press, Cambridge, MA, 1995, $293-322$.

[20] Rimányi R., Stevens L., Varchenko A., Combinatorics of rational functions and Poincaré-Birkhoff-Witt expansions of the canonical $U\left(\mathfrak{n}_{-}\right)$-valued differential form, Ann. Comb. 9 (2005), 57-74, math.CO/0407101.

[21] Silvotti R., On a conjecture of Varchenko, Invent. Math. 126 (1996), 235-248, alg-geom/9503016.

[22] Schechtman V., Terao H., Varchenko A., Local systems over complements of hyperplanes and the KacKazhdan conditions for singular vectors, J. Pure Appl. Algebra 100 (1995), 93-102, hep-th/9411083.

[23] Schechtman V., Varchenko A., Arrangements of hyperplanes and Lie algebra homology, Invent. Math. 106 (1991), 139-194.

[24] Talalaev D., Quantization of the Gaudin system, hep-th/0404153.

[25] Varchenko A., Multidimensional hypergeometric functions and representation theory of Lie algebras and quantum groups, Advanced Series in Mathematical Physics, Vol. 21, World Scientific Publishing Co., Inc., River Edge, NJ, 1995.

[26] Varchenko A., Critical points of the product of powers of linear functions and families of bases of singular vectors, Compos. Math. 97 (1995), 385-401, hep-th/9312119.

[27] Varchenko A., Bethe ansatz for arrangements of hyperplanes and the Gaudin model, Mosc. Math. J. 6 (2006), 195-210, 223-224, math.QA/0408001.

[28] Yuzvinsky S., Cohomology of the Brieskorn-Orlik-Solomon algebras, Comm. Algebra 23 (1995), 5339-5354.

[29] Zaslavsky T., Facing up to arrangements: face-count formulas for partitions of space by hyperplanes, Mem. Amer. Math. Soc. 1 (1975), no. 154. 